\title{
The Effect of Transcranial Electrical Stimulation on Athletic Performance Optimization: Systematic review, Meta- Analysis, and Proposing a Theoretical Model
}

\author{
Amin Amini*, Mohammad Vaezmousavi
}

Department of Artificial Intelligence and Cognitive Sciences, Imam Hossein University, Tehran, Iran

\section{A BSTRACT}

Introduction: Transcranial direct- current stimulation is a nerve modulation technique that induces low- intensity direct current to brain cells that stimulate or inhibit spontaneous neural activity. In recent decades, the use of electrical stimulation has been used as an effective method to improve the cognitive, psychological, and physical performance of athletes. In this study, using meta- analysis, the effectiveness of extracranial electrical stimulation on optimizing athletes' performance was investigated. Materials and Methods: The present study is a systematic review and meta-analysis research model. In the first phase of the study, all Persian and English studies on the effectiveness of Transcranial direct- current stimulation on athlete's performance were systematically reviewed $(\mathrm{N}=474)$, and among them, studies with meta- analysis criteria were reviewed by Meta- Analysis Basics and Applications $(\mathrm{N}=26)$. In the second stage, a specific intervention model for athletes was developed based on various components affecting performance. Results: The results showed the effect size (ES) of transcranial direct- current stimulation (Cohen's $\mathrm{d}=1.187$ ) is large according to Cohen's table (Cohen's $\mathrm{D}>0.8$ ). Therefore, transcranial direct- current stimulation improves the performance of athletes. In the second stage, the factors affecting the performance of athletes following transcranial direct- current stimulation were developed in the form of a specific intervention model for athletes. Conclusion: The findings of this meta- analysis showed a large ES for transcranial direct- current stimulation on athletic performance optimization.

*Corresponding Author: Amin Amini

Email:amini.a@ut.ac.ir 
تأثير تحريك الكتريكى فراجمجمهاى بر بهينهسازى عملكرد ورزشكاران؛ يك مرور نظاممند، فراتحليل

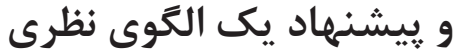

امين امينى"، سيد محمدكاظم واعظ موسوى

كروه هوش مصنوعى و علوم شناختى، دانشخاه امام حسين، تهران، ايران

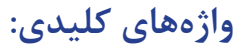

1- ادراك

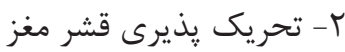

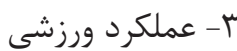

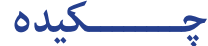

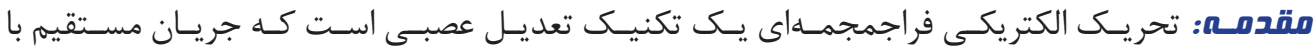

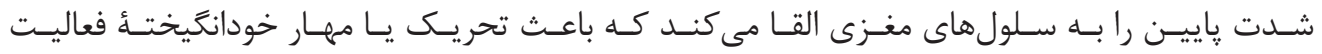

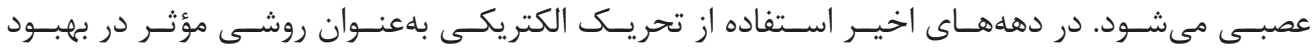

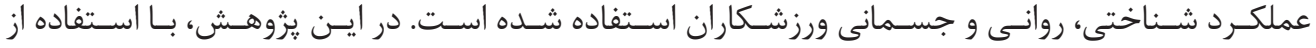

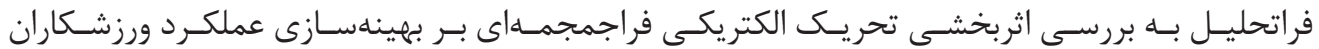

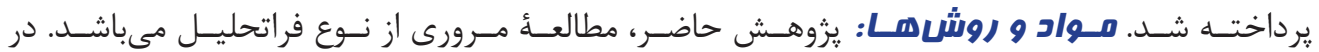

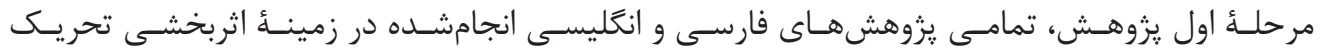

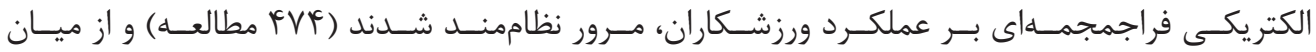

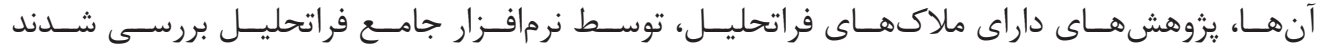

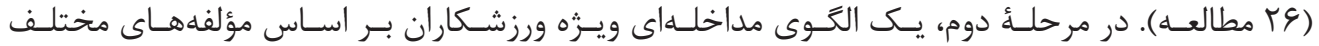

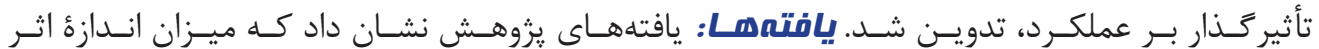

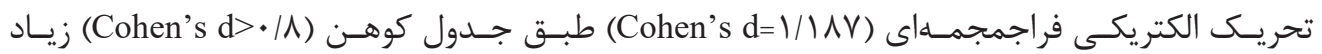

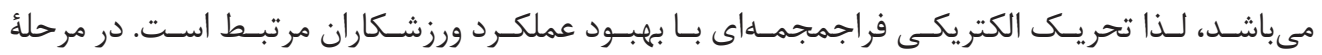

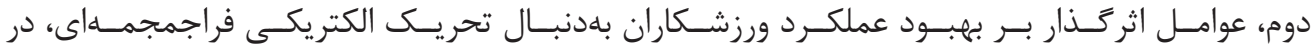

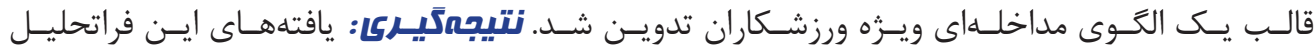

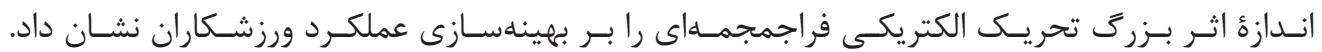




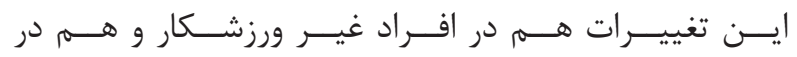

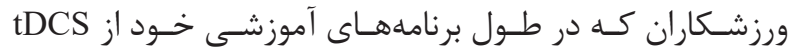

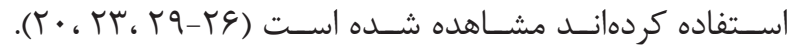

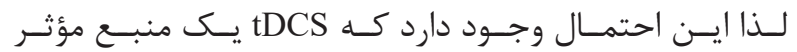

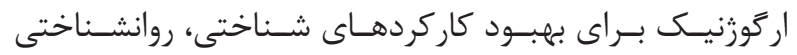

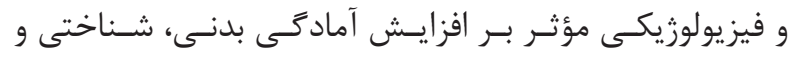

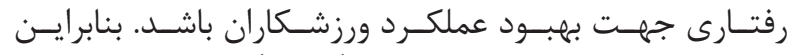

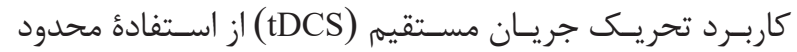

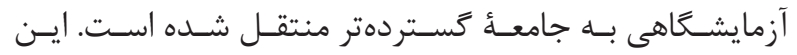

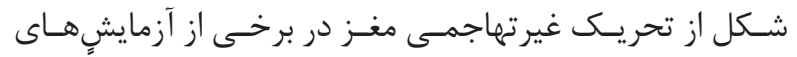

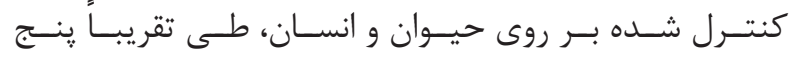

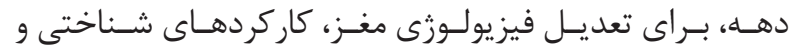

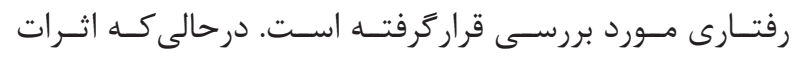

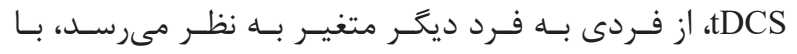

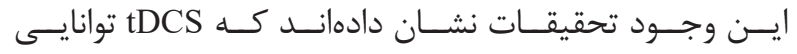

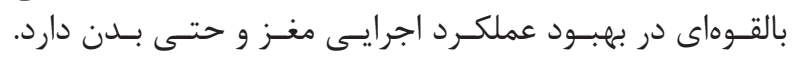

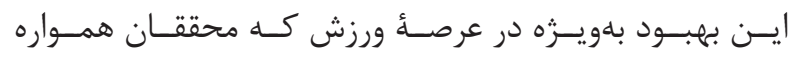

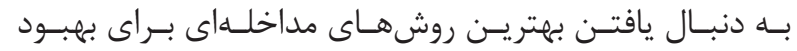

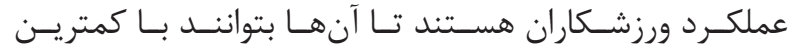

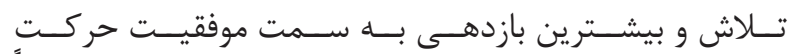

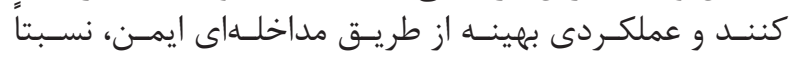

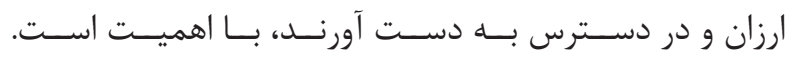

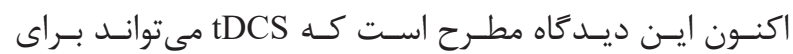

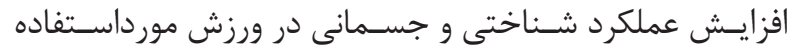

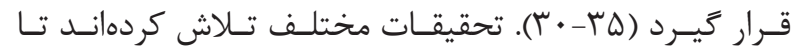

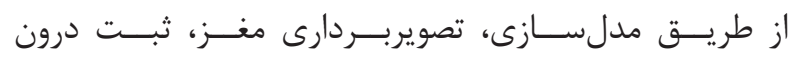

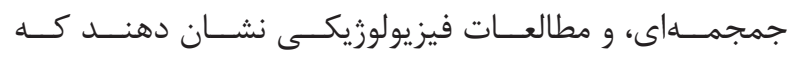

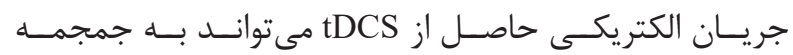

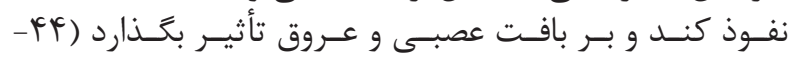

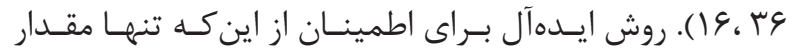

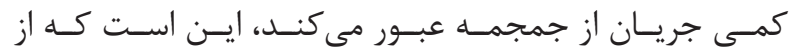

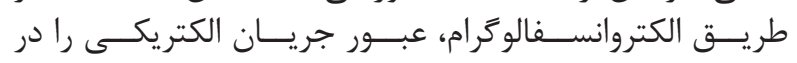

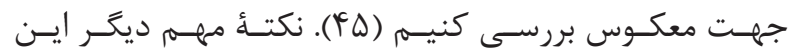

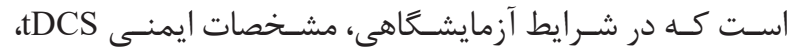

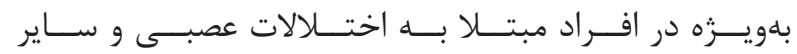

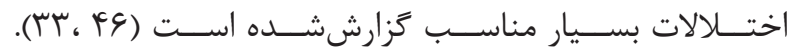

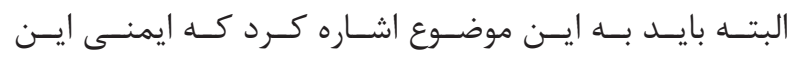

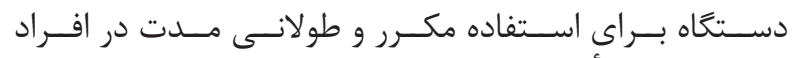

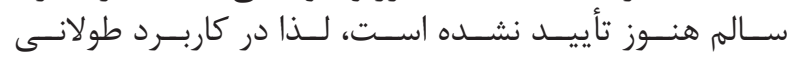

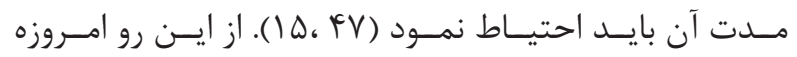

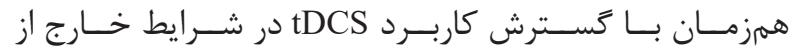

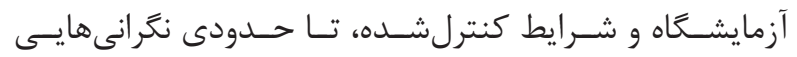

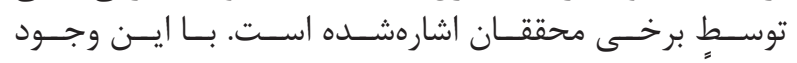

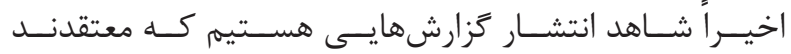

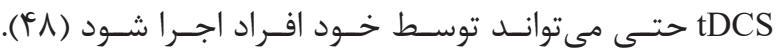

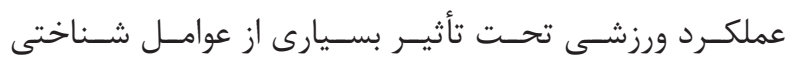

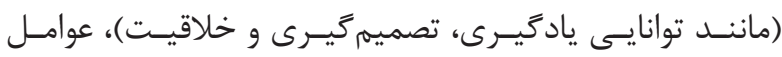

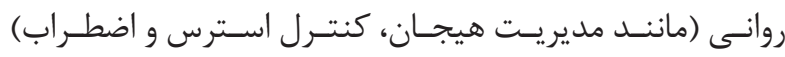

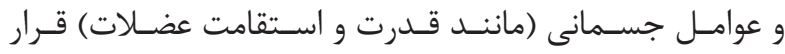

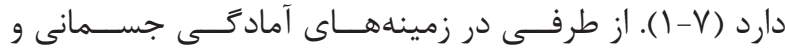

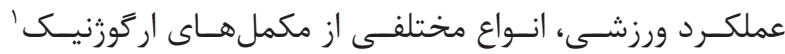

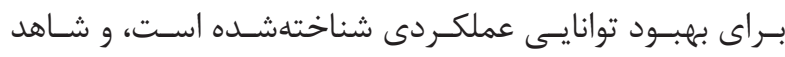

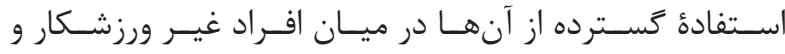

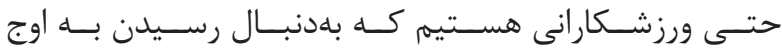

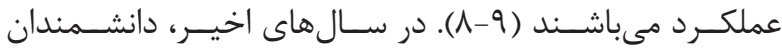

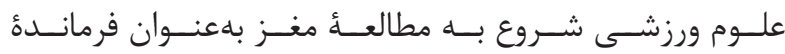

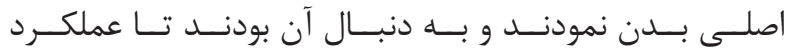

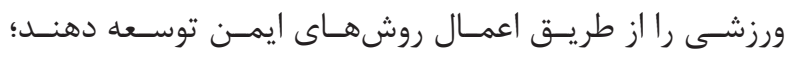

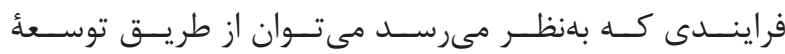

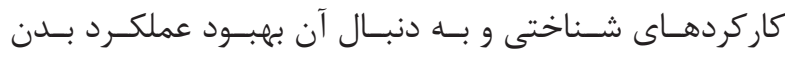

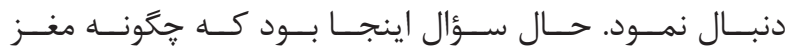

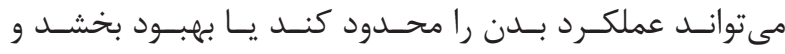

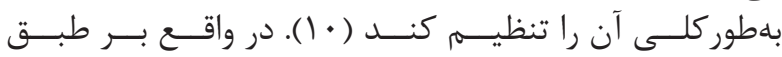

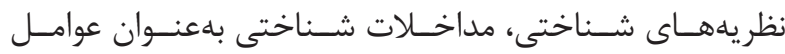

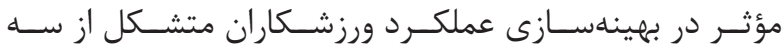

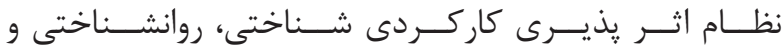

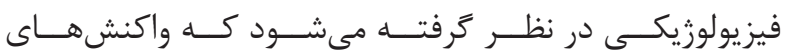

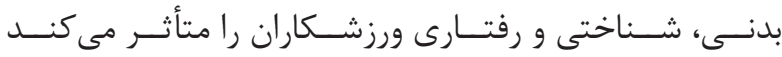

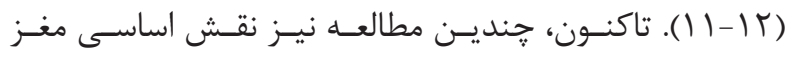

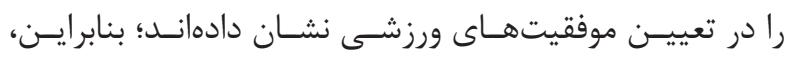

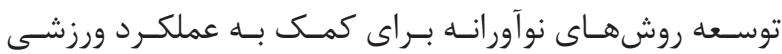

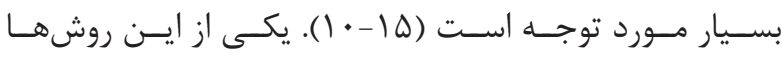

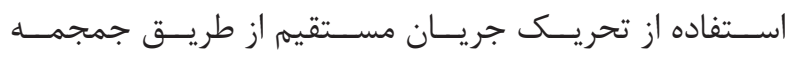
(tDCS)

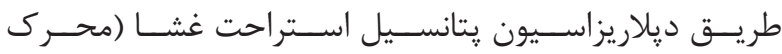

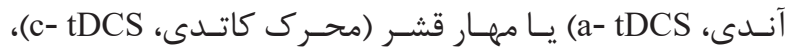

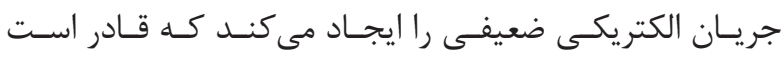

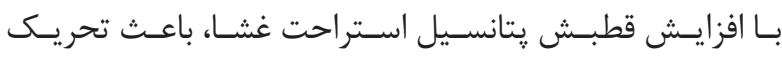

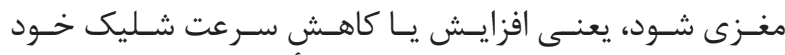

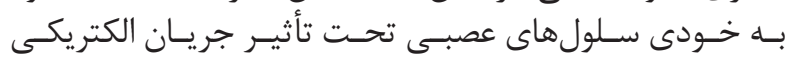

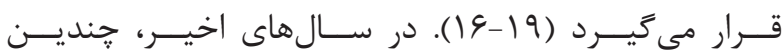

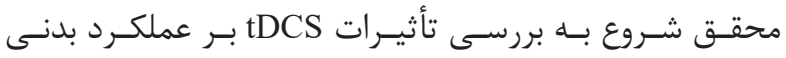

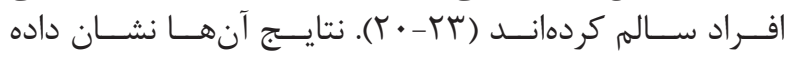

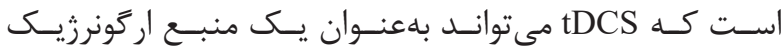

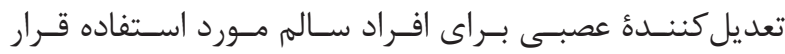

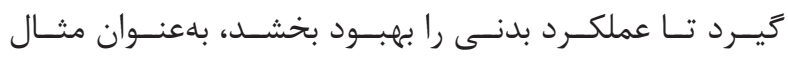

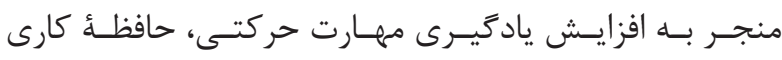

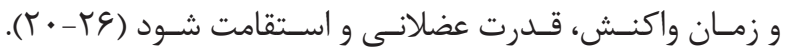

${ }^{1}$ Ergogenic 


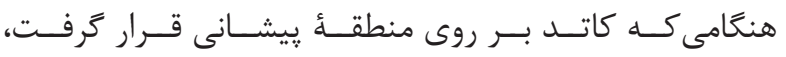

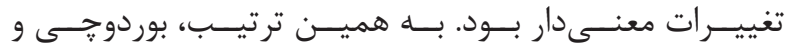

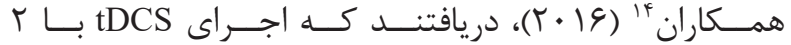

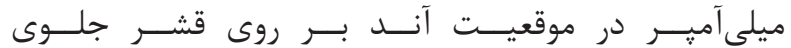

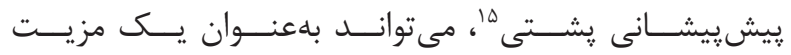

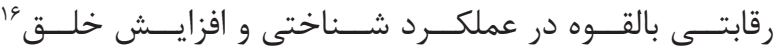

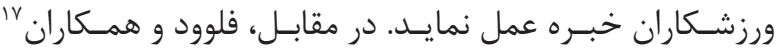
(T • IV)

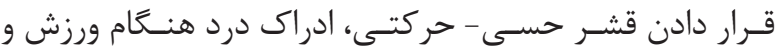

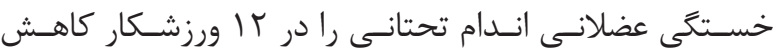

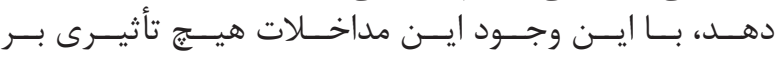

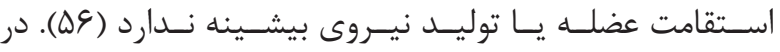

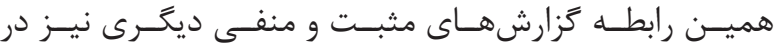

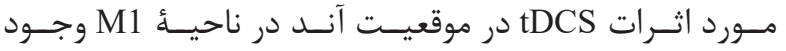

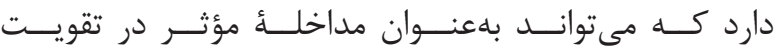

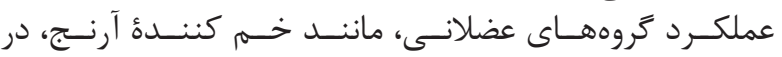

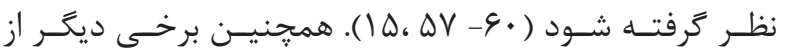

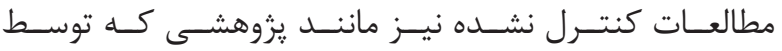

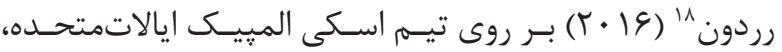

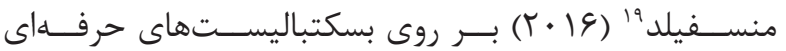
NBA

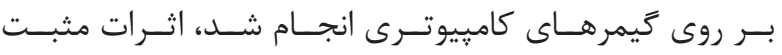
را نشـان دادنـد (TDCS

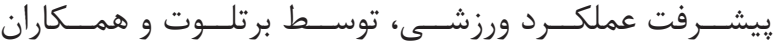

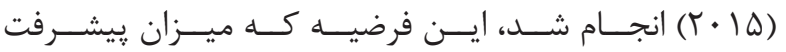

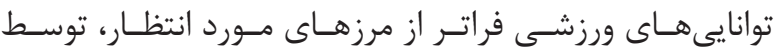

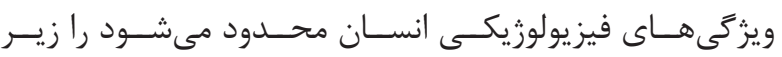

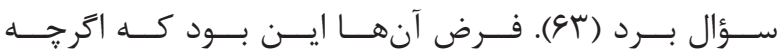

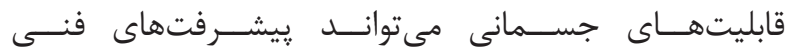

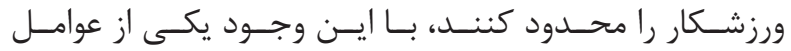

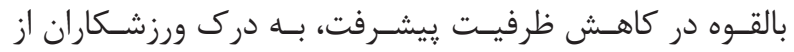

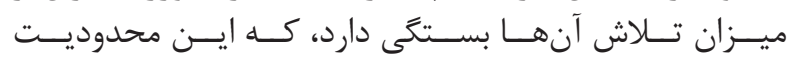

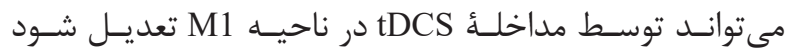

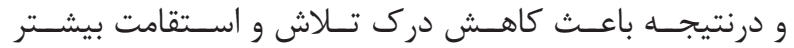

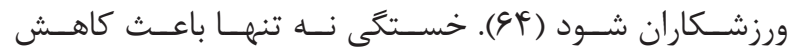

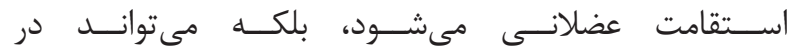

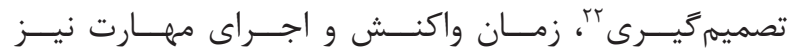

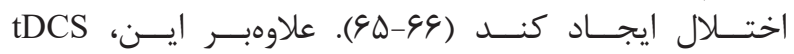

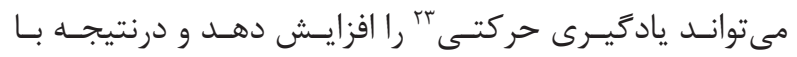

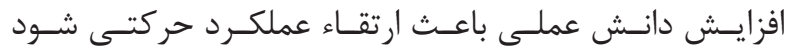

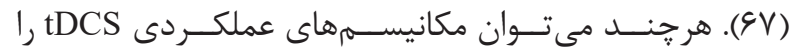

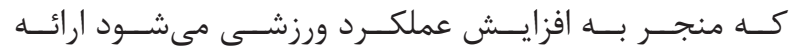

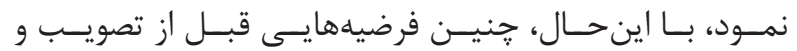

${ }^{2}$ Okano

${ }^{3}$ Anode

${ }^{4}$ Left Temporal Cortex

${ }^{5}$ Peak Power

${ }^{6}$ Clarke

${ }^{7}$ Perceptual-Learning Paradigm

${ }^{8}$ Object Detection

${ }^{9}$ Simulated Combat Environment

${ }^{10}$ Right Inferior Frontal Cortex

${ }^{11}$ Reduced Fatigue

${ }^{12}$ Improved Threat Detection

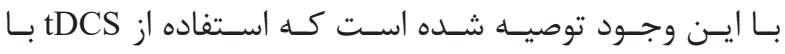

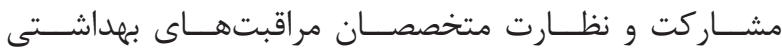

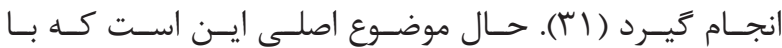

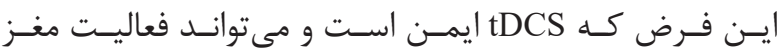

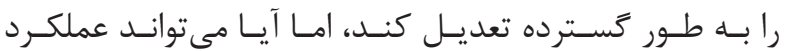

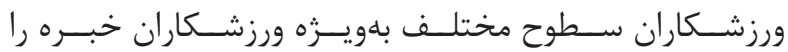

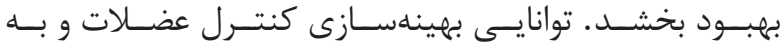

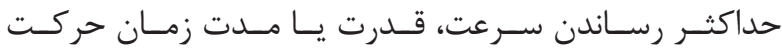

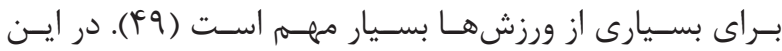

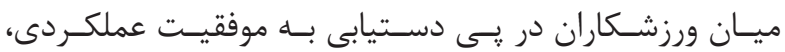

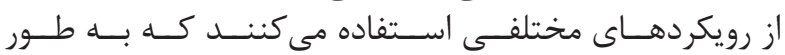

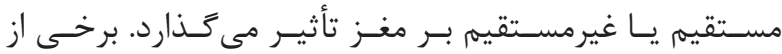

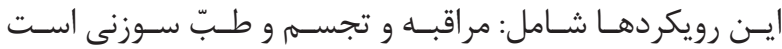

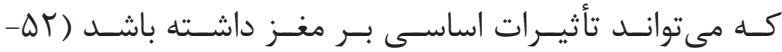

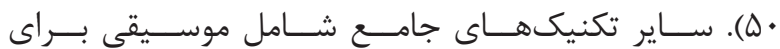

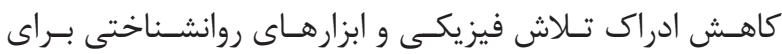

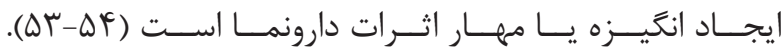

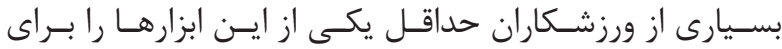

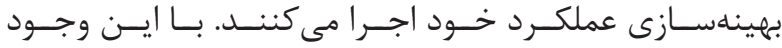

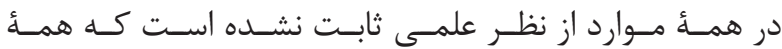

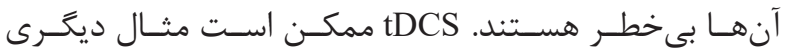

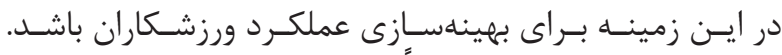

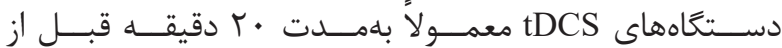

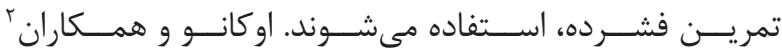

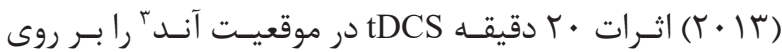

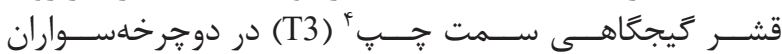

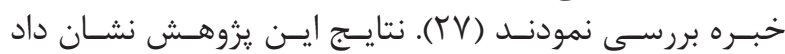

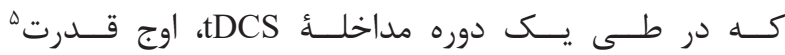

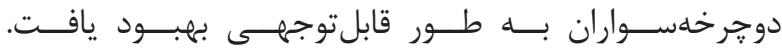

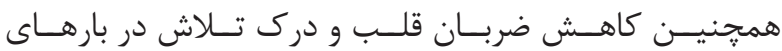

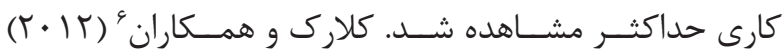

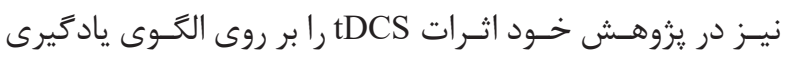

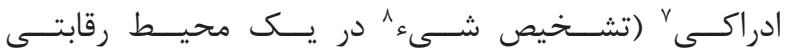

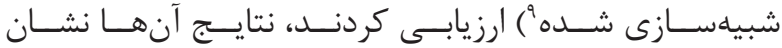

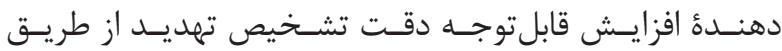

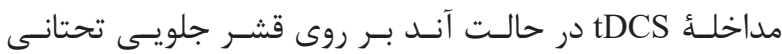

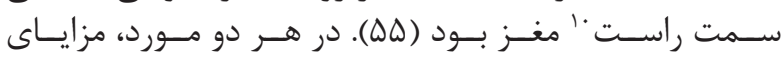

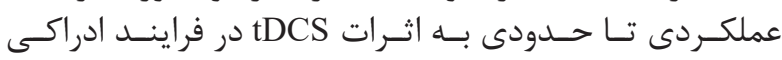

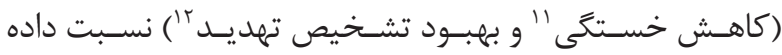

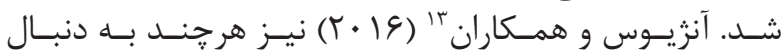

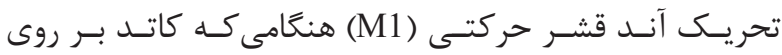

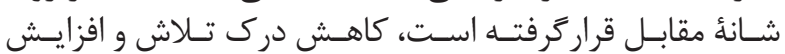

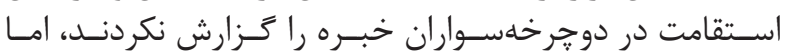

${ }^{13}$ Angius

${ }^{14}$ Borducchi

${ }^{15}$ Left Dorsolateral Prefrontal Cortex

${ }^{16}$ Mood

${ }^{17}$ Flood

${ }^{18}$ Reardon

${ }^{19}$ Mansfield

${ }^{20}$ Falcone And Parasuraman

${ }^{21}$ Jarrett

${ }^{22}$ Decision- Making

${ }^{23}$ Motor Learning 
حـذف مقـالات: مطالعـات در صورتـى وارد تحقيـق مىشـــند

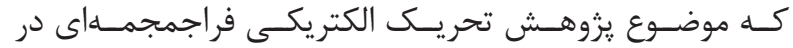

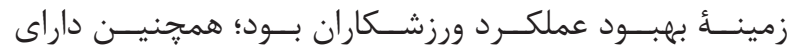

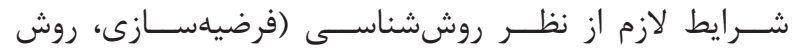

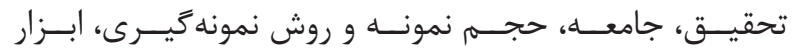

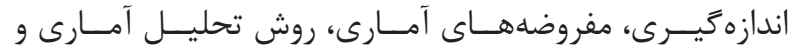

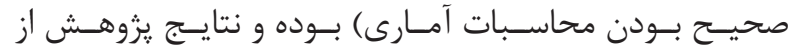

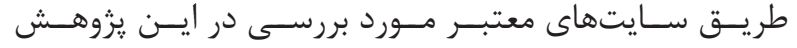

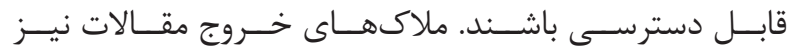

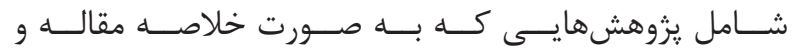

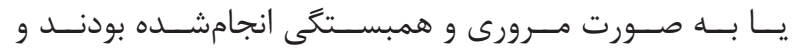

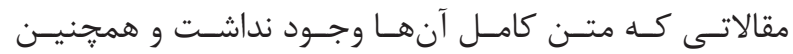

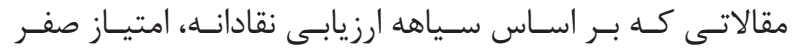

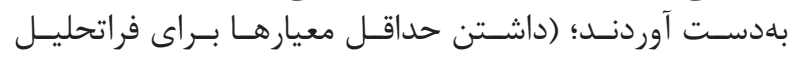

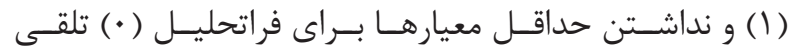

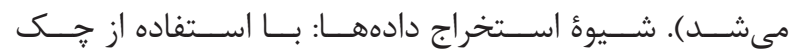

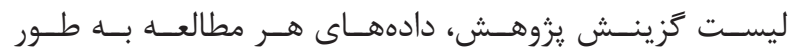

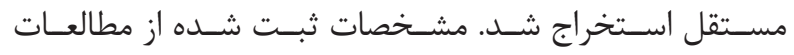

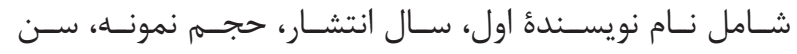

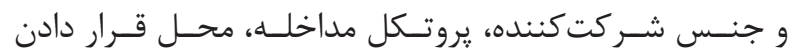

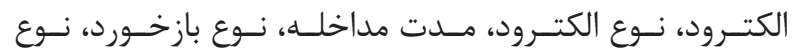

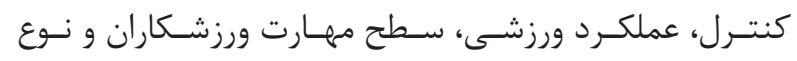

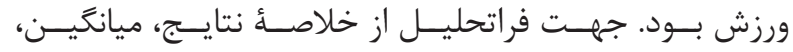

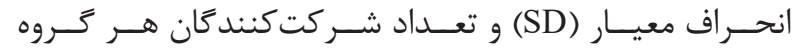

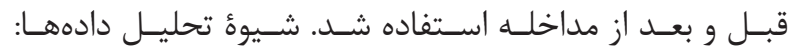

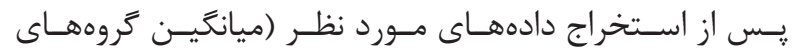

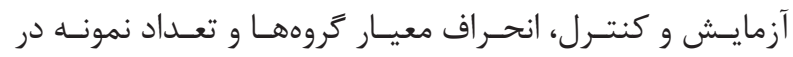

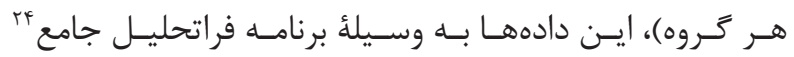

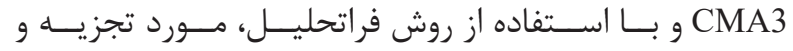

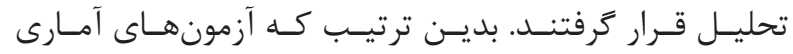

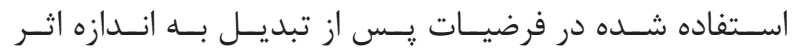

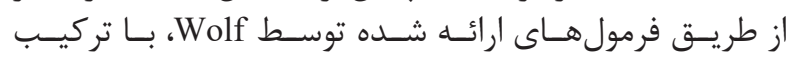

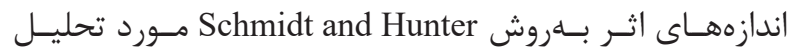

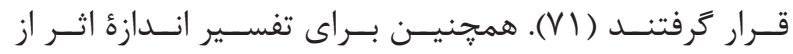

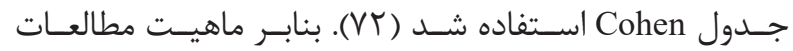

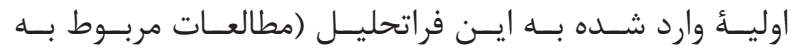

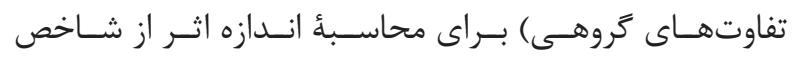

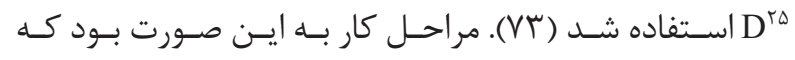

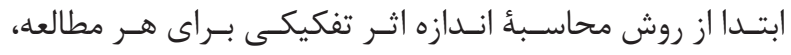

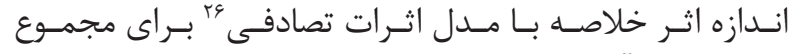

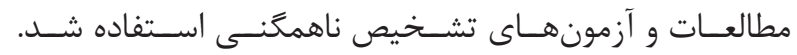

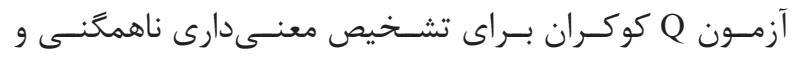

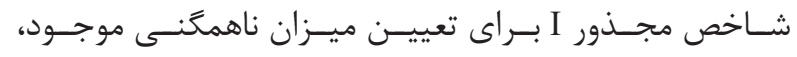

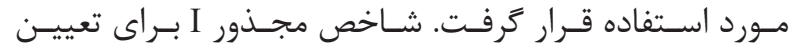

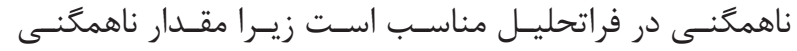

${ }^{24}$ Comprehensive Meta-Analysis

${ }^{25}$ Standardized Mean Difference (SMD)

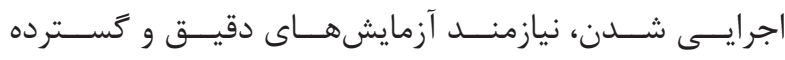

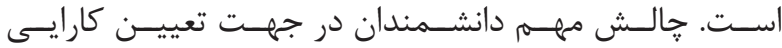
دDCS

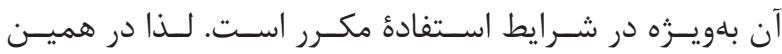

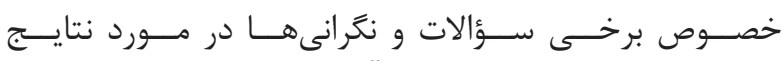

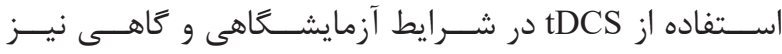

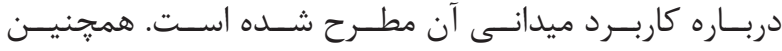

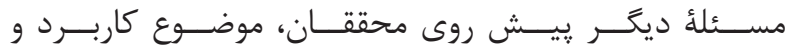

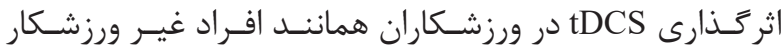

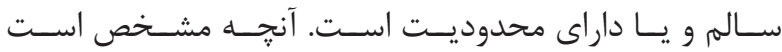

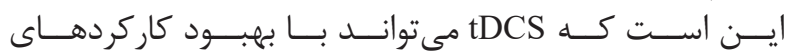

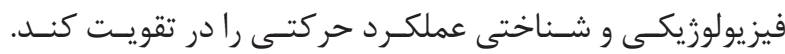

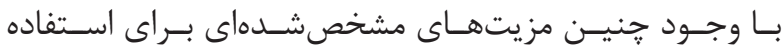

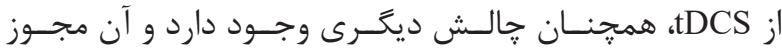

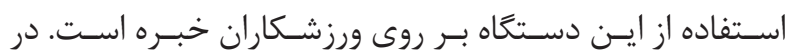

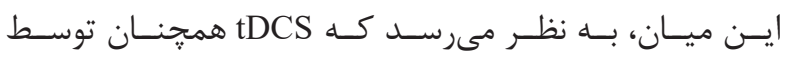

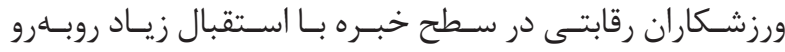

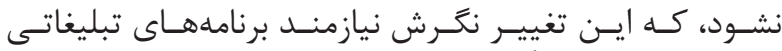

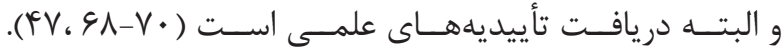

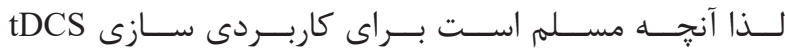

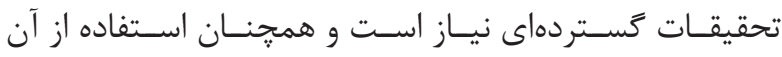

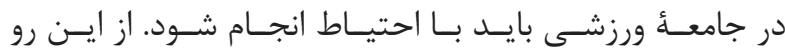

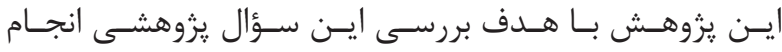

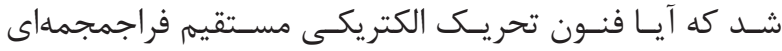

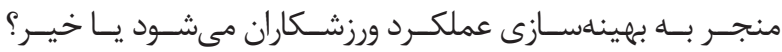

مواد و روشها - (- ماد

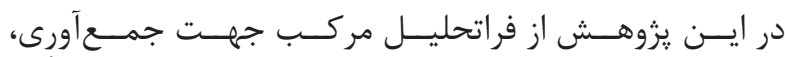

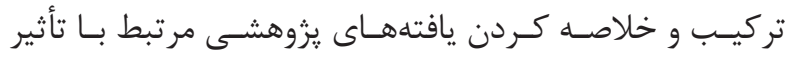

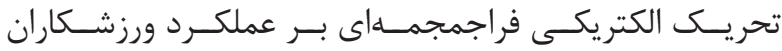

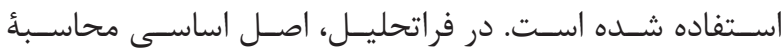

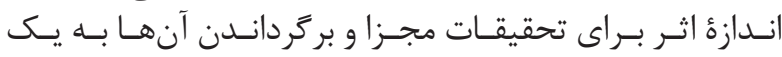

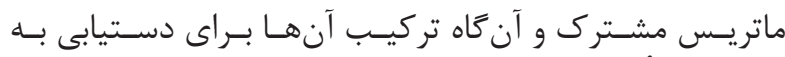

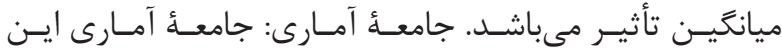

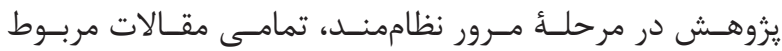

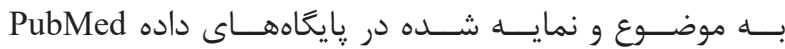
Cochrane da-،Scopus ، Science Direct، Google Scholar، Magiran، SID، Psych info، tabase

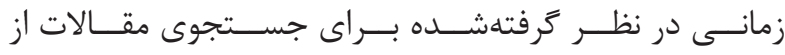

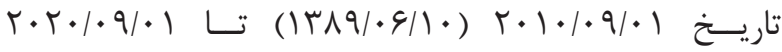

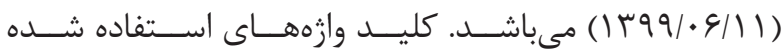

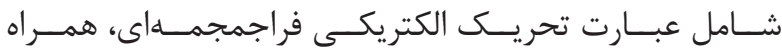

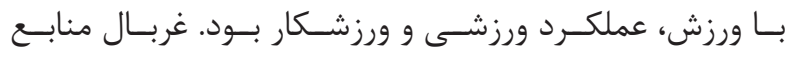

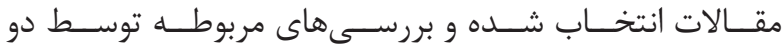

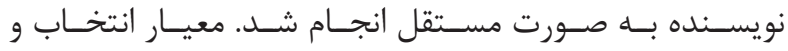

${ }^{26}$ Random Effects Model 
روش الكويابـى معسـادلات سـاختارى (SEM) اعمــال گرديــد.

بافته ها

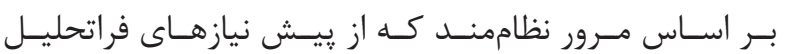

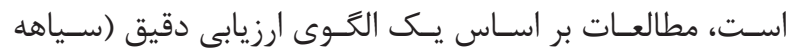

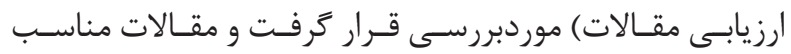

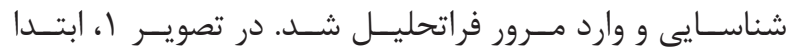

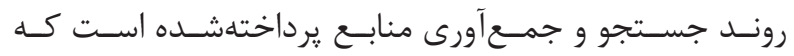

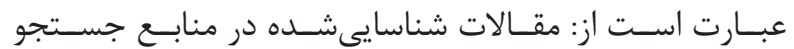
(FVF)

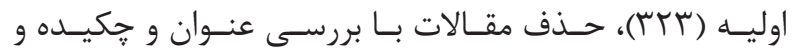

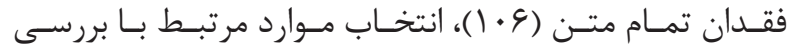

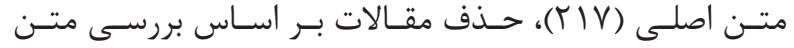

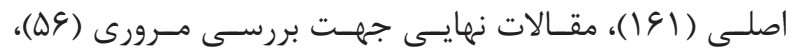

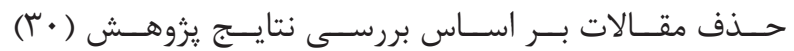

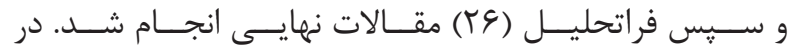

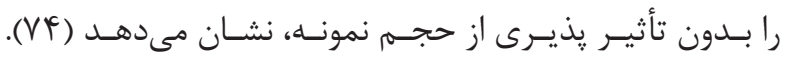

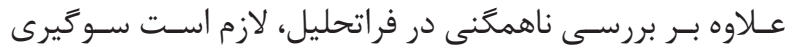

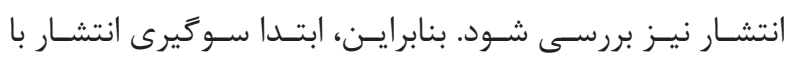

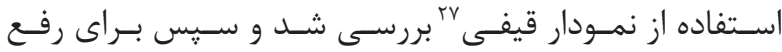

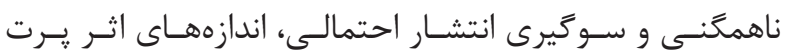

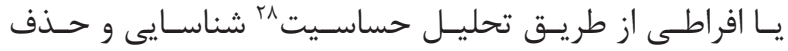

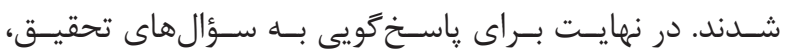

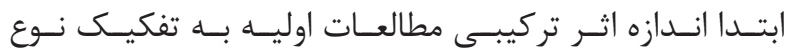

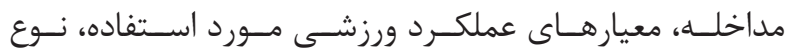

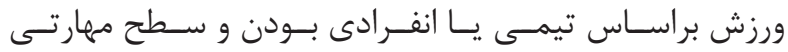

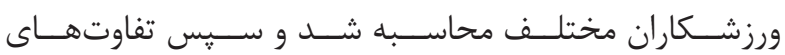

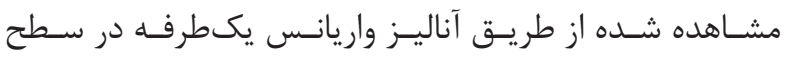

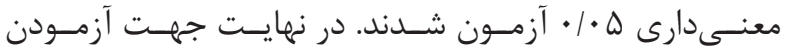

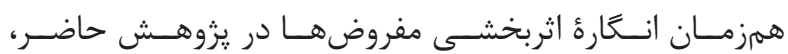

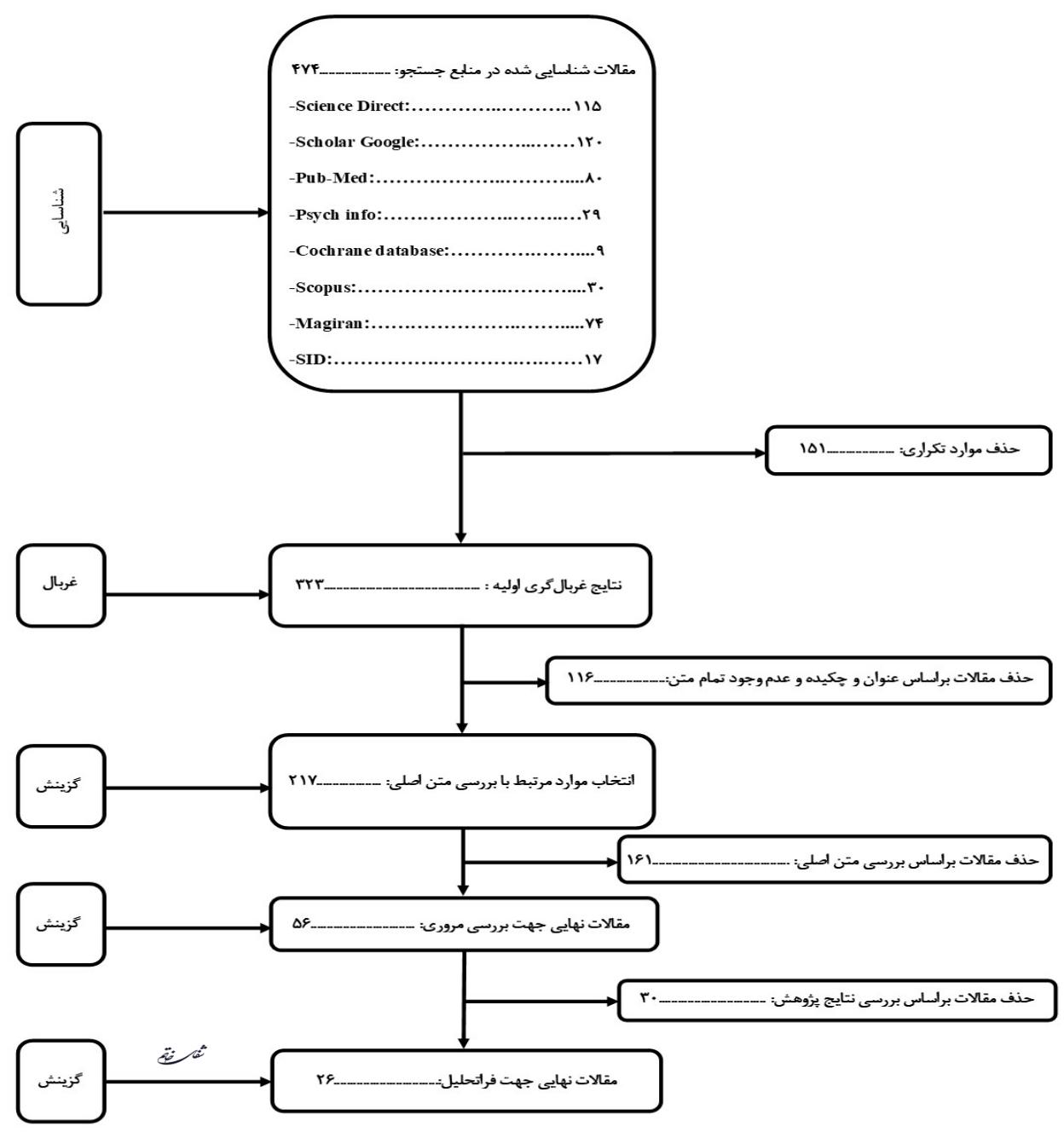




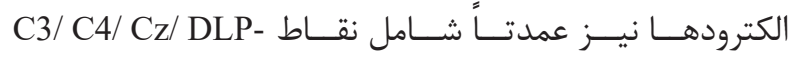
(FC/ Fp2/ FP1/ T3/ F3/ F4/ P4/ Oz ارزيابسى عملكـرد ورزشـكاران شـامل زمــان واكنـش، گتشـتاور

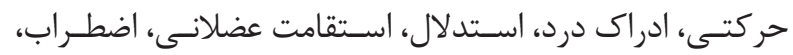

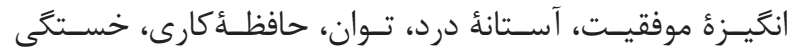

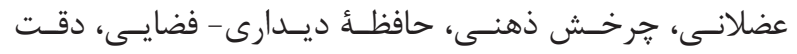

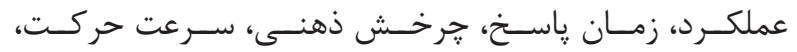

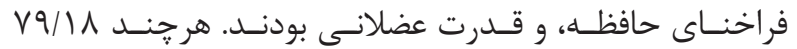

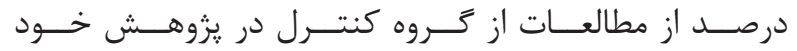

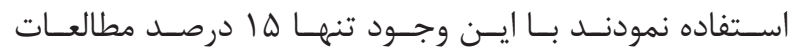

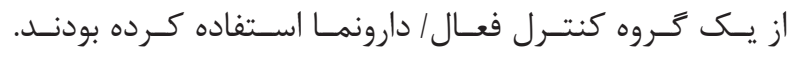

\section{نتايج مرور نظاممند}

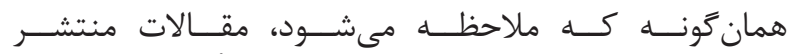

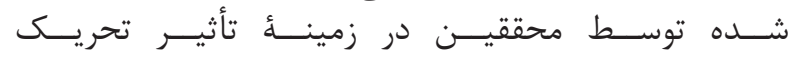

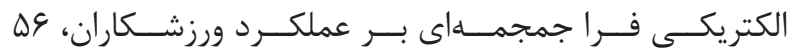

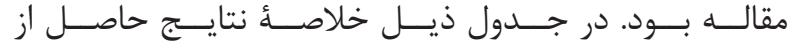

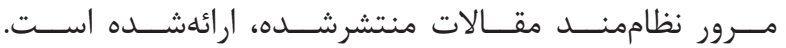

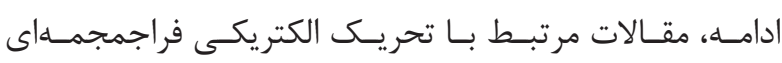

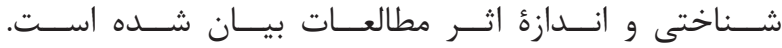

\section{ويثز تَى هاى مطالعات}

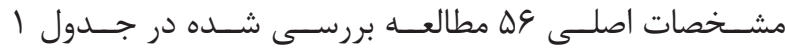

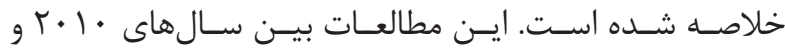

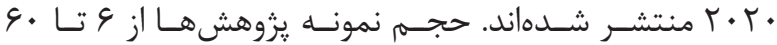

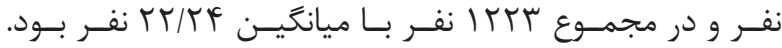

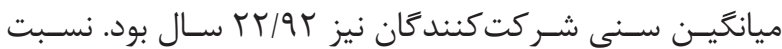

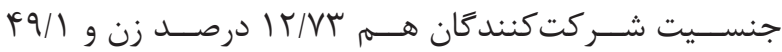

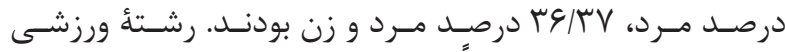

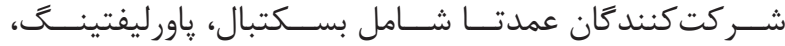

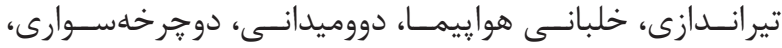

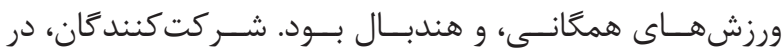

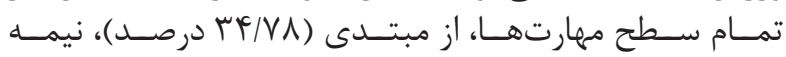

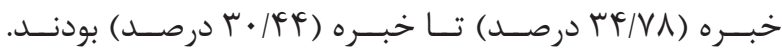

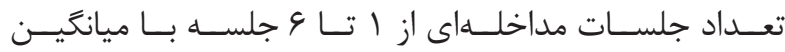

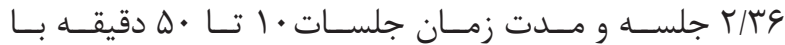

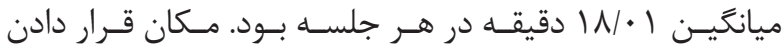

جدول ا- مشخصات مقالات منتشرشده توسط محققين در زمينأ تحريك الكتريكى فراجمجمهاى بر بهينهسازى عملكرد ورزشكاران

\begin{tabular}{|c|c|c|c|c|c|c|c|c|c|c|c|c|c|}
\hline 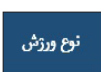 & سطع ورزثكار & الرئيى عملكرد & نمزئ & | لمون & تموناد & شترايط & مالكاته & جالسات & 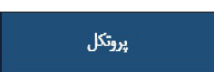 & المتروة & مكان الكتروه & L & 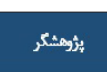 \\
\hline مهاني & هبتدى & 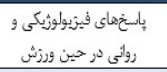 & مرد & $r r$ & $\Delta 1$ & كبا كتروه & r. & 1 & الكترود أندى قشئر ريشيشيشانى & أندال و & DLPFC & r.r. & $\begin{array}{c}\text { Wertheim } \\
\& \text { et al.(vo) }\end{array}$ \\
\hline |دويرخهـوارى & خبره & قدرت واتقالت عذالمى & مرد & $\pi$ & $\Delta$. & كتثرل & $\Delta$. & r & 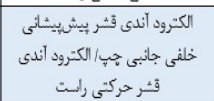 & 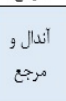 & C4/DLPFC & r.r. & $\begin{array}{l}\text { Grandperin } \\
\text { \& et al.(199) }\end{array}$ \\
\hline تكولندو & نيمه خبره & سرعت ضريه & مرن & $r \cdot 10$ & 19 & كبتركرة & 10 & 1 & 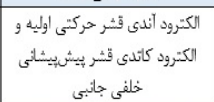 & 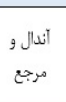 & $\begin{array}{l}\mathrm{C} 3 / \mathrm{CA} / / \\
\text { DLPFC }\end{array}$ & $r .19$ & $\begin{array}{l}\text { Mesquita } \\
\text { \& et al.(YY) }\end{array}$ \\
\hline كوتبال هنبال & نيمه خبره & زمان واكش & مرد و & 11 & $* 8$ & كتثراكرو & r. & 1 & 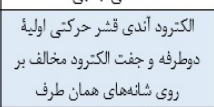 & أندال و & $\mathrm{C} 3 / \mathrm{C}_{4}$ & $r .19$ & $\begin{array}{c}\text { Seidel } \\
\text { \&et al. }(Y A)\end{array}$ \\
\hline همثانى & مبتدى & قدرت عضلاكى & مرن & if & 10 & كباكروه & r. & 1 & الكترود أندى قشتر بيشريشانى & أندال و & DLPFC & $r .19$ & $\begin{array}{c}\text { Lattari } \\
\text { \& et al.(Y9) }\end{array}$ \\
\hline همثانى & نيمه خبره & قدرت إيزومتريك & مرد و رن & if & ir & كباكروه & r. & 1 & الكترود أندى قشبر حركى اولية & أندال و & C3 & r.19 & $\begin{array}{c}\text { Frazer } \\
\text { \& et al. }(\Lambda \cdot)\end{array}$ \\
\hline دويرخهـوارى & خبره & التقامت عشالى & مرد & rorr & re & كبترلروه & r. & $r$ & 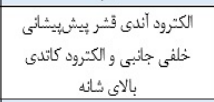 & كأثنال، & DLPFC & $r .19$ & $\begin{array}{l}\text { Holgado } \\
\text { \& et al. (N) }\end{array}$ \\
\hline دووميدالى & خبره & سرعت دويلن و تنغير & مرن & rq & 1. & كثترو كثره & r. & 1 & الكترود أندى قشر حركتى اوليه & 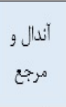 & $\mathrm{C} 3 / \mathrm{C} 4 / \mathrm{Cz}$ & $r .19$ & $\begin{array}{c}\text { Park \& } \\
\text { et al. (Ar) }\end{array}$ \\
\hline همثانى & نيمه خبره & خستى إيزوكيتيك & مرد & r & $r$. & كبثرل باكوه & 10 & 0 & الكتروه أندى قدر تميورال جيب & أندال و & T3 & $r .19$ & $\begin{array}{l}\text { Ciccone } \\
\text { \& et al.(Alt) }\end{array}$ \\
\hline همثانى & نيمه خبره & تصميم:ميري:ماي حركتى & مرد & r & $r$. & كثترل & r. & r & 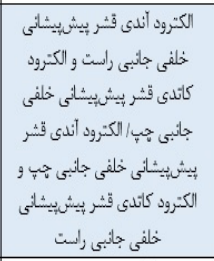 & كأندال. & DLPFC & $r .19$ & $\begin{array}{c}\text { Ota } \\
\text { \& et al. }(\Lambda f)\end{array}$ \\
\hline | دوجرخهسوارى & نيمه خبره & بازمارى يالسخ & مرن & r & ir & كبت كتروه & r. & 1 & 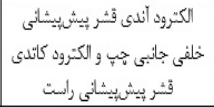 & أندال و & $\begin{array}{c}\mathrm{DLPFC} / / \\
\text { Fp2 }\end{array}$ & r. 19 & $\begin{array}{l}\text { Angius } \\
\text { \& et al. (A } \Delta)\end{array}$ \\
\hline 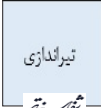 & خبره & ركورد تيراندازى & مرد & $r .19$ & 18 & كبثرل & r. & r & 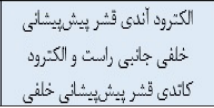 & كائدال. & DLPFC & r. 19 & $\begin{array}{c}\text { Kamali } \\
\text { \& et al. }(\wedge \varphi)\end{array}$ \\
\hline
\end{tabular}




\begin{tabular}{|c|c|c|c|c|c|c|c|c|c|c|c|c|c|}
\hline & & & & & & & & & جائبى جنج & & & & \\
\hline باورليفتيثة & خبره & استدلال، حافظظه و توانايى & مرد و & 4 & ir & كبترل باتروه & ir & r & 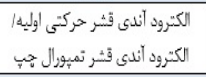 & مرجع أندال و & $\mathrm{C} 3 / \mathrm{C}_{4} / \mathrm{T} 3$ & $r .19$ & $\begin{array}{c}\text { Kamali } \\
\text { \& et al.(AV) }\end{array}$ \\
\hline دوجرخهـوارى & خبره & 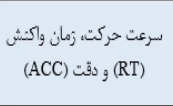 & مرن & TEIT & 9 & كترول بدوه & r. & $\Delta$ & 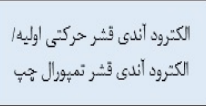 & 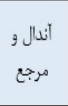 & $\mathrm{C} 3 / \mathrm{C}_{4} / \mathrm{T} 3$ & $r \cdot 19$ & $\begin{array}{l}\text { Huang } \\
\text { \& et al. }(\Lambda)\end{array}$ \\
\hline همثانى & 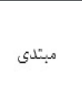 & ازريبابي عصبي - عضلانى & مرد & ro/9 & ir & كثمرل & 1. & 9 & 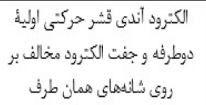 & كاتدال & $\mathrm{C} 3 / \mathrm{C} 4$ & $r .11$ & $\begin{array}{l}\text { Angius } \\
\text { \& et al. (Tr) }\end{array}$ \\
\hline شنا & خبره & ضريان قلب و حالت & مرد & if & $\wedge$ & كتبرل & r. & 1 & دالكترود أندى قشر حركتى اوليئ & مرجع أندال و & $\mathrm{C} 3 / \mathrm{C} 4$ & $r \cdot 11$ & $\begin{array}{l}\text { Valenzuela } \\
\text { \& et al. (19) }\end{array}$ \\
\hline 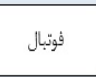 & خبره & قدرت عضلات جهار سر ران & مرد و ور & rf & r. & كبا كرول & 10 & $f$ & الكترود أندى قشر حركتى اوليه & أندال و & $\mathrm{C} 3 / \mathrm{C} 3$ & $r .11$ & $\begin{array}{c}\text { Vargas } \\
\& \text { et al. }(9 \cdot)\end{array}$ \\
\hline دوجرخهـوارى & خبره & استقامت عضالثى & مرد & rell & rq & كثترل & r. & $r$ & الكترود أندى قُشر بيشيشيشانى & كاتدال & DLPFC & $r \cdot 11$ & $\begin{array}{c}\text { Holgado } \\
\text { \& et al.(१1) } \\
\end{array}$ \\
\hline ل يثبال & خبره & قدرت عضلاثى ايزوومتريك & زن & 1911 & 19 & كثياترول & r. & $r$ & 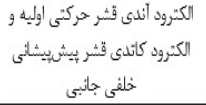 & مرجغ & $\begin{array}{l}\mathrm{C} 3 / \mathrm{C} 4 / \\
\mathrm{DLPFC}\end{array}$ & $r .1 \mathrm{~V}$ & $\begin{array}{c}\text { Hazim } \\
\text { \& et al. }(9 Y)\end{array}$ \\
\hline همثانى & 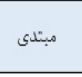 & قدلدرت عضلاتى & 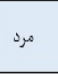 & rV & 11 & كثيرل & r. & 1 & 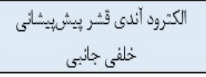 & مرجع أندال و & DLPFC & $r \cdot 11$ & $\begin{array}{c}\text { Lattari } \\
\text { \& et al. }\left(\left(^{9}\right)\right.\end{array}$ \\
\hline همثانى & مبتدى ل & 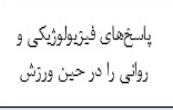 & 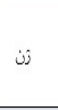 & $19 / 9$ & ir & 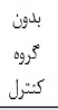 & r. & 1 & دالكترود أندى قشر حركتى اوليئ & أندال و & $\mathrm{C} 3 / \mathrm{C} 4$ & $r .1 \mathrm{~V}$ & $\begin{array}{l}\text { Okano } \\
\text { \& et al.(9Y) }\end{array}$ \\
\hline همثانى & 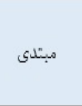 & أنططافيذيرى مفصل & مرد & $r r$ & 1 & كثترل بدوه & 1. & 1 & الكترود أندى قشر حسى - حركتى & كاتدال & $\mathrm{Cz}$ & r.IV & $\begin{array}{l}\text { Mizuno } \\
\text { \& et al. (9 } 9 \text { ) }\end{array}$ \\
\hline همثانى & 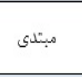 & قدات عضلاتى & 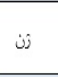 & $r \Delta$ & 10 & كثياتروه & r. & r & الكترود أندى قُشر بيشيشائى & مرجع أندال و & DLPFC & $r \cdot r \cdot$ & $\begin{array}{c}\text { Lattari } \\
\text { \& et al.(Tr) }\end{array}$ \\
\hline همثانى & 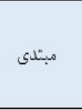 & حداكثر انقباضات ارادى & 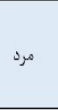 & rf & $f f$ & كثترل & r. & 1 & 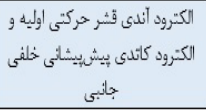 & مرجغ & $\begin{array}{l}\mathrm{C} 3 / \mathrm{C} 4 / \\
\mathrm{DLPFC}\end{array}$ & $r .1 \mathrm{~V}$ & $\begin{array}{l}\text { Radel } \\
\text { \& et al.(१४) }\end{array}$ \\
\hline همثَانى & 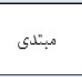 & جابكى دست & زن - ت & $r \Delta$ & ri & كثبرل & 10 & 1 & الكترود أندى قشر حركتى اوليه & مرجع أندال و & $\mathrm{C} 3 / \mathrm{C} 4$ & $r .1 \mathrm{~V}$ & $\begin{array}{c}\text { Pixa } \\
\text { \& et al. }(99)\end{array}$ \\
\hline هostiانى & نيمه خبره & 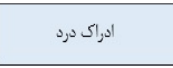 & مرد و ت ون & ri/9 & ir & كبتر كروه & r. & 1 & الكترود أندى قشر حركى اوليه & أندال و & $\mathrm{C} 3 / \mathrm{C} 4 / \mathrm{Cz}$ & $r .1 \mathrm{~V}$ & 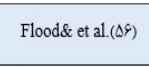 \\
\hline هostiانى & مبتدى مي & 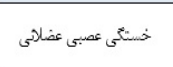 & مرن & rA & rr & كابتر كتروه & 1. & r & جب الكترود أندى قشر حركتى اولئئ. & مرجع أن و & $\mathrm{C} 3$ & $r .19$ & $\begin{array}{c}\text { Abdelmoula \& et al. } \\
(\Delta \Lambda)\end{array}$ \\
\hline همخانى & 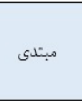 & قدرت عضلانى & 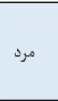 & rfif & 1. & كتبرل & r. & r & الكترود أندى قشر حركتى اوليئ & 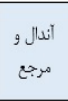 & $\mathrm{C} 3 / \mathrm{C} 4$ & $r .19$ & $\begin{array}{l}\text { Lattari } \\
\text { \& et al.(YI) }\end{array}$ \\
\hline هماثانى & 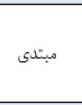 & إستقاهت عضلانى & زَن & r & 1. & كثترون & r. & r & الكترود أندى قشر حركتى اوليئ & أندال و & $\mathrm{C} 3 / \mathrm{C} 4$ & $r .19$ & $\begin{array}{c}\text { Lattari } \\
\text { \& et al. }(\Gamma \cdot)\end{array}$ \\
\hline دوجرخهسوارى & خبره & استقامت عضلادى & مرد & r 910 & 9 & كثترل & r. & r & الكترود أندى قشر حركتى اوليه & أندال و & C3 & $r .19$ & $\begin{array}{l}\text { Barwood } \\
\text { \& et al.(9V) }\end{array}$ \\
\hline هنديال & نيمه خبره & استقامت عضلانى & 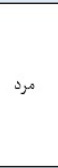 & r/10 & 9 & كثترل كروه & 1. & r & 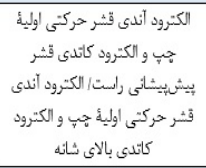 & 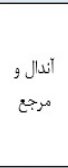 & $\mathrm{C} 3 / \mathrm{FP} 2$ & $r .19$ & $\begin{array}{c}\text { Angius } \\
\text { \& et al.(9८) }\end{array}$ \\
\hline مانمانى & نيمه خبره & عملكرد ايزوكيثتيك عضلاثى & مر مرد و & r & 19 & كبتر كتروه & $r$. & $\Delta$ & الكترود أندى قشر تميورال سمت & أندال و & T3 & $r .19$ & $\begin{array}{l}\text { Magalhães } \\
\text { \& et al.(१9) }\end{array}$ \\
\hline
\end{tabular}




\begin{tabular}{|c|c|c|c|c|c|c|c|c|c|c|c|c|c|}
\hline 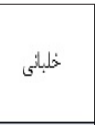 & خبره & حافظة كارى & مرد & $r$ & $\pi$ & كثترل بأروه & r. & ${ }^{f}$ & 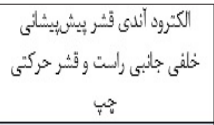 & 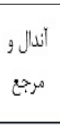 & $\mathrm{DLPFC} / \mathrm{C} 3$ & $r .19$ & $\begin{array}{l}\text { Choe } \\
\text { \& et al. }((\cdot \cdot \cdot)\end{array}$ \\
\hline ل موجرخسوار & نيمه خبره & حلاكثر توان هوازى & مرد & rol & 1. & كثترل كبرون & r. & r & الكترود أندى قُش تميوزول جيب & مرجع & T3 & $r .10$ & $\begin{array}{l}\text { Okano } \\
\text { \& et al. (YY) }\end{array}$ \\
\hline همثانى & 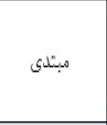 & الفزايش تحريكيذينيى & si & $r \lambda$ & rf & كثترل & 10 & 9 & 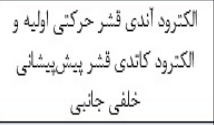 & أندال و & $\begin{array}{l}\mathrm{C} 3 / \mathrm{CA} / \\
\mathrm{DLPFC}\end{array}$ & $r .10$ & $\begin{array}{l}\text { Hendy } \\
\text { \& et al. }((\cdot) \cdot 1)\end{array}$ \\
\hline | دوجرخهوارى & خبره & الستقات عضلآى & 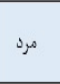 & $r$ & 11 & كبترل بروه & ir & r & الكترود أندى قثر حركنى اوليه & كأنوال، & $\mathrm{C} 3 / \mathrm{CH}_{4}$ & $r .10$ & $\begin{array}{l}\text { Vitor-Costa } \\
\text { \& et al.(.94) }\end{array}$ \\
\hline همثانى & 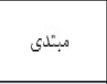 & قلمدت عضلاتى & 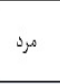 & $\pi$ & if & كبتر كوره & r. & 1 & الكترود أندى قشر حركى اوليه & أندال و & $\mathrm{C} 3 / \mathrm{C} 4$ & $r .10$ & $\begin{array}{l}\text { Montenegro } \\
\& \text { et al. }(1 \cdot \Gamma)\end{array}$ \\
\hline همثانى & ن ن بيه خبره & ادراك خسنى & 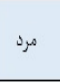 & rq & Ir & كبترل & 10 & 1 & الكترود أندى قثر حركى ولئية & أندال و & C3 & $r .10$ & $\begin{array}{l}\text { Ayres Montenegro } \\
\text { \& et al. }(1 \cdot \Gamma)\end{array}$ \\
\hline مهثاني & نبيه خبره & ادراك درد & مرد & If & 9 & بلدون & 10 & $r$ & 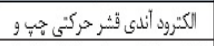 & أندال و & $\mathrm{C} 3 / \mathrm{FP} 2$ & 5.10 & Angius \& \\
\hline & & & & & & كثترل & & & 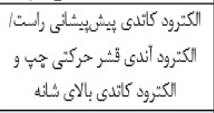 & مرجع & & & et al $(1 \cdot f)$ \\
\hline همثانى & 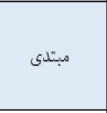 & الثطافيانيري قشر حركتى | & مرن & rq & r. & كثترل باكوه & r. & $r$ & 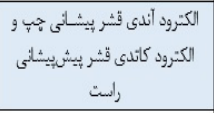 & كائدال. & $\mathrm{F} 3 / \mathrm{FP} 2$ & r.lr & $\begin{array}{c}\text { Hendy } \\
\text { \& et al. }(1 \cdot \bullet)\end{array}$ \\
\hline هostrivي & هبتدى & 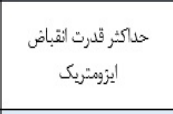 & مرن مرد & rA & $r$. & كثترل باكوه & 1. & 1 & راست و الكترود كاتدى روى ثشانئ. & 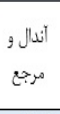 & $\mathrm{C} 4$ & r.1r & $\begin{array}{c}\text { Kan } \\
\text { \& et at. }\left(Q^{\circ}\right)\end{array}$ \\
\hline همثانى & 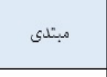 & خدنّى عصبي -عضلاتى & مرد & $\pi$ & 10 & كبترل بأروه & 1. & 1 & الكترود أندى قشتر بيشيريشائى & أندال و & DLPFC & r.1r & $\begin{array}{l}\text { Muthalib } \\
\text { \& et al al.(.). }\end{array}$ \\
\hline لهمثانى & نيمه خبره & 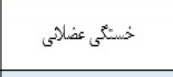 & مرد & $r \Lambda$ & 11 & كبترل كبروه & r. & 1 & 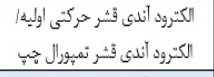 & أندال و & $\mathrm{C} 3 / \mathrm{T3}$ & $r .14$ & $\begin{array}{r}\text { Williams } \\
\text { \& et al. }(94)\end{array}$ \\
\hline همثانى & ن نيمه خبره & قدرت ايزومتريك & مرد & ryir & ir & كبتر بروه & 1. & 1 & الكترود أندى قثر حركتى الولية & كأندال، & C3 & $r .14$ & $\begin{array}{l}\text { Lampropoulou et al. } \\
\qquad(1.9)\end{array}$ \\
\hline همثاني & مبتدى جائباز & اضطراب & 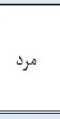 & rYir & if & كثترل & r. & $r$ & 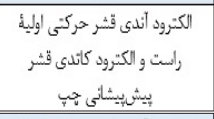 & مرجع أندال و & $\mathrm{C} 4 / \mathrm{FP} 1$ & $1 \mp 99$ & أرسطو و همكاران (7. (1) \\
\hline همثانى & مبتلدى جائباز & كتثرل باسهر & 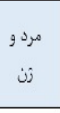 & ro & fo & كبثرل باكوه & r. & $r$ & 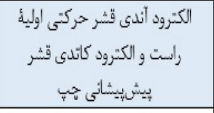 & 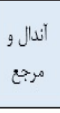 & $\mathrm{C} 4 / \mathrm{FP} 1$ & 1999 & عربي و همكاران (A - () \\
\hline بسكتبال & 'نيه خبره & أزالد بسكتبال & مرن & $\pi$ & is & كثترل & 10 & 9 & 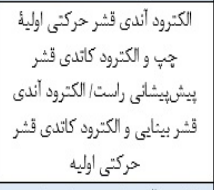 & 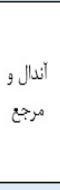 & $\begin{array}{c}\mathrm{C} 3-\mathrm{FP} 2 / \\
\mathrm{Oz}-\mathrm{Cz}\end{array}$ & 1799 & 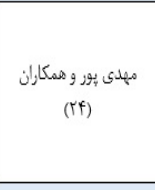 \\
\hline لمثمانى & مبتدى & 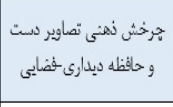 & 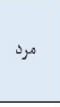 & $r / / 6$ & of & كثترل باكوه & 10 & 1 & 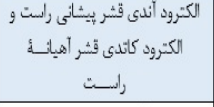 & مرجع أندال & F4 / P4 & 1 ז91 & 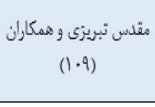 \\
\hline هostrivى & مبتلدى جائباز & زمان واكثش & 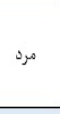 & $r V / d$ & If & كثبرل & r. & $r$ & 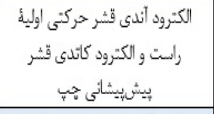 & مرجع الن و & $\mathrm{C} 4 / \mathrm{FP} 1$ & $1+94$ & أرسطو و همكاران (. (11) \\
\hline همثانى & 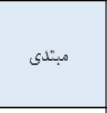 & اجراى قدرتى و استقامتى & زن & $r$. & 1. & كبترل بلدون & 10 & $r$ & 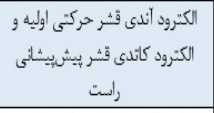 & مرجع & $\begin{array}{l}\mathrm{C} 3 / \mathrm{C} 4 / \\
\mathrm{FP} 2\end{array}$ & 1 ז9A & نظرى بيردوستى و \\
\hline 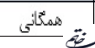 & ميتدى . & توانايى تصويرسازي & مردو & TF/O & 9. & باكتروه & 10 & $\Delta$ & الكترود أندى قشتر يثشانى راست و و & أندال و & $\mathrm{F} 4 / \mathrm{P} 4$ & Irav & باورى كاتب و همكاران \\
\hline
\end{tabular}




\begin{tabular}{|c|c|c|c|c|c|c|c|c|c|c|c|c|c|}
\hline & & & is & & & كثترل & & & 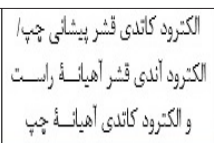 & مرجغ & & & (III) \\
\hline رزمى & نبيه خبرها & كثنل باسهر & مرد & if & r. & كثرل بأروه & 10 & $r$ & 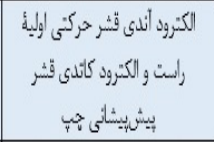 & أنال & $\mathrm{C} 4 / \mathrm{FP} 1$ & I $199 \mathrm{~V}$ & دلفي وهكاران (III) \\
\hline همثانى & مبتدى جانباز & عالظظه كارى & مرد & $r / r$ & If & كثنرل & 10 & $r$ & 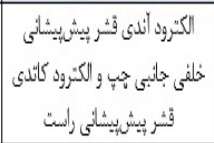 & مرجع & $\mathrm{F} 3 / \mathrm{FP} 2$ & IFAY & ارسطو و همكاران ("آل) \\
\hline مهانى & هبتلى ل & توجها تاتخغلى & مرن & $\mathrm{rF} / \mathrm{Q}$ & is & كثترل بوره & r. & 1 & 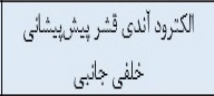 & أندال و & DLPFC & 1999 & 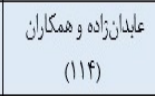 \\
\hline هذأنى & مبثلى & حالظطك كارى وزمان واكثش & مرد & $1 / \Delta$ & r. & كثترل & r. & r & 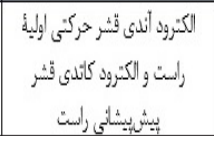 & مرجع & $\mathrm{C} 4 / \mathrm{FP} 2$ & 1999 & زماني و ومكاران (ه') ) \\
\hline
\end{tabular}

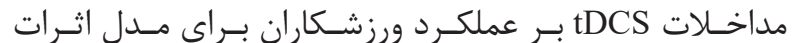

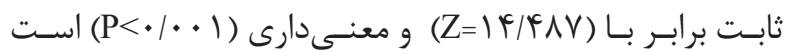

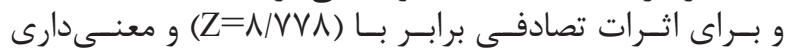

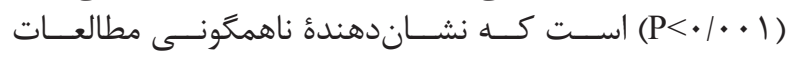

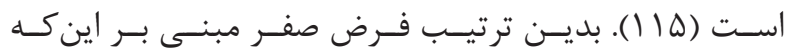

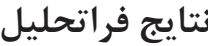

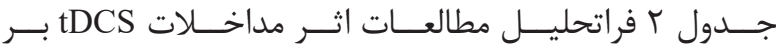

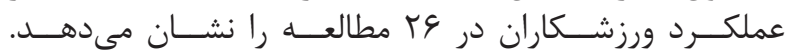

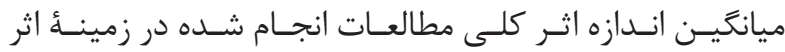

جدول r - اندازء اثر تحريك الكتريكى فراجمجمهاى بر بهينهسازى عملكرد ورزشكاران

\begin{tabular}{|c|c|c|c|c|c|c|c|c|c|c|c|c|c|c|}
\hline & & & 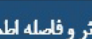 & & & 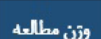 & & تمترل & & & tDC & & سال & 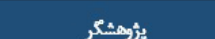 \\
\hline & & 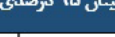 & & & & 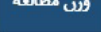 & $\mathbf{N}$ & SD & Mean & $\mathrm{N}$ & SD & Mean & قسال & ? \\
\hline & & & & & $\cdot / 189[-\cdot / 499, \cdot / 099]$ & $f / 1 \gamma \wedge$ & ro & 1995 & $\Delta f I f f$ & rq & $\Delta \wedge 19$ & $\Delta \Delta \cdot 9$ & $\mathrm{Y}$ & Vim \\
\hline & & & & & $\cdot / \Delta 99[-\cdot / r q \cdot, \cdot / V) \cdot]$ & $f / 199$ & $r \Delta$ & $\cdot / 14$ & $\cdot / \mathrm{V}$ & rq & $\cdot / 1 r$ & $\cdot / V T$ & T.P. & Wertherm et al. (VQ) \\
\hline & & & & & $f / f A V[r / \cdot V F, \Delta / r q r]$ & $\cdot / F \wedge$ & $\wedge$ & $1 / 0$ & $r \Delta / \Delta$ & 1 & $\Delta / 9$ & IV/f & & \\
\hline & & & & & I/NAV $[-\cdot / \cdot F q, Y / \cdot r V]$ & $1 / 199$ & $\wedge$ & $r / 4$ & $1 f / 0$ & 1 & $\pi / 1$ & $11 / 1$ & 5.19 & Kamali et al $\mathrm{cuv}$ \\
\hline & & & & & $|/ f r|[-\cdot / r T \cdot, Y / \cdot f V]$ & $\cdot / 9 \ldots$ & 9 & $f \pi T / \Delta$ & rFag & 9 & $90 \mathrm{~g} / \mathrm{f}$ & riff & 然 & 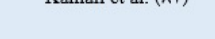 \\
\hline & & & & & $1 / f 9 r[-. / r \Delta r, r / .9 f]$ &.$/ 194$ & 9 & $r / \Delta$ & $19 / 1$ & 9 & $f / T$ & $10 / 9$ & & \\
\hline & & & & & $r / V V \Lambda[1 / \cdot \mid f, r / r \cdot . \cdot]$ & $1 / \cdot \Delta f$ & 1. & $r / 0$ & $19 / 4$ & 1. & $f / T f$ & $\Delta T / T F$ & $r .19$ & Park et al. (AT) \\
\hline & & & & & $\cdot / 1 \wedge \&[-\cdot / \lambda r V, 1 / \cdot \mid r]$ & l/frf & 9 & TNYA & $\triangle 9 N T$ T & 9 & $r q / V r$ & $\Delta \varphi \Delta / T \Delta$ & & \\
\hline & & & - & & $1 / f M[-\cdot / \pi r q, 1 / 9 V \Lambda]$ & $1 / T \wedge \Delta$ & 9 & .1 .9 & $\cdot / 11$ & 9 & $\% \cdot \Delta$ & .94 & $r .19$ & Huang et al. $(\Lambda \Lambda)$ \\
\hline & & & & & $\cdot / \operatorname{ATf}[-\cdot / \Delta f), \mid / T r \Delta]$ & $1 / 4+1$ & 9 & $\cdot \%$ &.$/ 90$ & 9 & $\% \cdot r$ & .199 & & \\
\hline & & & & & $r / V r g[-\cdot / r \cdot r, \mid / \lambda r r]$ & $r / / \Delta T$ & 10 & $\pi T / 1$ & TrVE & 10 & $r \Delta / \varphi$ & trfO & 5.19 & Otgetal $(\Delta f)$ \\
\hline & & & & & $f / 9 \lambda 1[1 / f f 1, r / r) \cdot]$ & I/fFY & 10 & $11 / 9$ & $1 F 9 / 14$ & 10 & $\Delta / 9$ & $194 \pi 1$ & 1 & Gid et dil. (AI) \\
\hline & & & & & $\cdot / \Delta 19[-\cdot / \Delta 9 \cdot, \mid / \cdot \cdot \Delta]$ & $1 / 9 \Delta 9$ & ir & $f r / \Delta$ & $r$ rq/f & ir & rNq & TrN.O & $r \cdot 11$ & Holgado et al.(१।) \\
\hline & & & & & $1 / \Lambda \cdot r[-\cdot \cdot \cdot 9 \Lambda, r / r r \Delta]$ & $\cdot|\lambda \Delta|$ & 9 & $4 / 1$ & $11 / 1$ & 9 & $r / 9$ & $\mid f / T$ & $r .11$ & Angius et al.(Tr) \\
\hline & & & & & $-\cdot / 1 \cdot 9[-\cdot / 19 \cdot, \cdot / 79 \Delta]$ & $1 / \mathrm{VrV}$ & 11 & $1 / 9$ & Nf & 11 & $\cdot / 4$ & $\Lambda$ & & \\
\hline & & & & & $1 / 9+\Lambda[-\cdot / / r V, 1 / \Delta \Lambda \Lambda]$ & $1 / 995$ & 11 & $V \pi / 1$ & $|r V| \mid$ & 11 & $9 V / T$ & $199 / \Delta$ & & \\
\hline & & & & & $f / f G r[1 / 111, r / \Delta 99]$ & $1 / 945$ & 10 & rAV/9 & $1 \Delta f 1 / 1$ & 10 & $f \triangle N q$ & $t r f \cdot / t$ & $r .1 \mathrm{r}$ & Lattari et al. $(\Upsilon \varphi)$ \\
\hline & & & & & $\cdot / / 99[-\cdot / 94 f, \cdot / Y M \Lambda]$ & $r / f \Delta \Lambda$ & 10 & TAV/8 & $10 f 1 / 1$ & 10 & rTo & $1019 / f$ & & \\
\hline & & & & & $\cdot / K r \wedge[-\cdot / 9 \pi T, \cdot / V 99]$ & $r / f \Delta V$ & 10 & $1 / 98$ & fiar & 10 & $1 / \cdot 1$ & $f / \Lambda$ & & \\
\hline & & & & & $T / T / f[-\cdot / \cdot 9 V, 1 / \Delta 91]$ & $r / T \Delta \Lambda$ & 10 & $1 / 98$ & fiar & 10 & $1 / \pi 9$ & $91 \pi$ & & \\
\hline & & & & & $\cdot / 9 \cdot 1[-\cdot / \Delta \Delta V, 1 / \cdot \Delta \cdot]$ & $1 / 9 \Delta r$ & IT & $t / V I$ & rH/QT & ir & $1 / V r$ & tfifA & & \\
\hline & & & & & $1 / r \Delta 9[-* / r 91,1 / \pi T V]$ & $1 / 9 \cdot r$ & ir & $r / r$ & $\mid \Delta / r$ & ir & f/. 9 & IVITo & r.IV & Flood et al. $(\Delta \varphi)$ \\
\hline & & & & & $r / 1 \cdot 9[-.1 .9 r, 1 / V F r]$ & $1 /$ VAs & ir &.$/ 99$ & $\cdot \pi 4$ & ir & $1 / \pi 1$ & $1 / \pi r$ & & \\
\hline & & & & & $\cdot / f f v[-\cdot / 919, \cdot / 91 \Delta \Delta]$ & $1 / 9 \Delta 9$ & IT & $f F / T \Delta$ & $1 \cdot \cdot / r$ & ir & $\mathrm{HT/NT}$ & $9 \pi / V$ & & \\
\hline & & & - & & $\cdot / \pi \cdot[-\cdot / V V f, \mid / \cdot V q]$ & $1 / f v 1$ & 9 & $11 f$ & irt & 9 & Irf & 191 & $r .19$ & Angius et al. (१४) \\
\hline & & & - & & $\cdot / V Y 1[-\cdot / \Delta 9 \Delta, 1 / T 9 \Lambda]$ & $1 / f \Delta 1$ & 9 & $11 f$ & ivr & 9 & 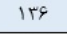 & $r 19$ & & \\
\hline & & & - & & $\cdot / V \& V[-\cdot / \Delta \mid r, 1 / 1 V \cdot]$ & $1 / 1 \times 9$ & 11 & $\mathrm{Ft/ \textrm {V }}$ & $|+9| 9$ & 11 & $\Delta T / \Lambda$ & $19 \pi / 4$ & $r .19$ & Abdelmoula et al. $(\Delta \Lambda)$ \\
\hline & & & & & $f / I f \cdot[-\cdot / r \Delta r, r / V A r]$ & $\cdot /$ /AT & 1. & $9 \pi / 0$ & $V \neq \Delta$ & 1. & $r \Delta / \Delta$ & ATO & $5 \cdot 19$ & Saleo et a \\
\hline & & & - & & I/VTT $[-\cdot / 1 / T, / / V G H]$ & $1 / 4 \pi$ & 1. & $r \cdot / \Delta$ & THNO & 1. & $1 f / \Delta$ & ror & r & Sales et di. \\
\hline & & & & & $1 / \cdot 91[-\cdot / 4 \cdot 1 \mathrm{~g} 1 / / \mathrm{V} \cdot]$ & $1 / 095$ & 1. & $19 / 1$ & $r .1$ & 1. & $r q / 9$ & $r 11 / r$ & & \\
\hline & & & & & $1 / \cdot T[-\cdot / F T \Delta, 1 / T O T]$ & $1 / 09 V$ & 1. & fo & VTt/ & 1. & $\mathrm{~V} / \mathrm{\theta}$ & $V \Delta 1 / f$ & $r \cdot 10$ & Okano et al. (r \\
\hline & & & & & $1 / 9 \Delta[-\cdot / \% a r, 1 / 4 \lambda \gamma]$ & $1 / \Delta 9$. & 1. & $r \Delta / f$ & ITO & 1. & $\Delta T / T$ & $1 \mp V / \Delta$ & & \\
\hline
\end{tabular}




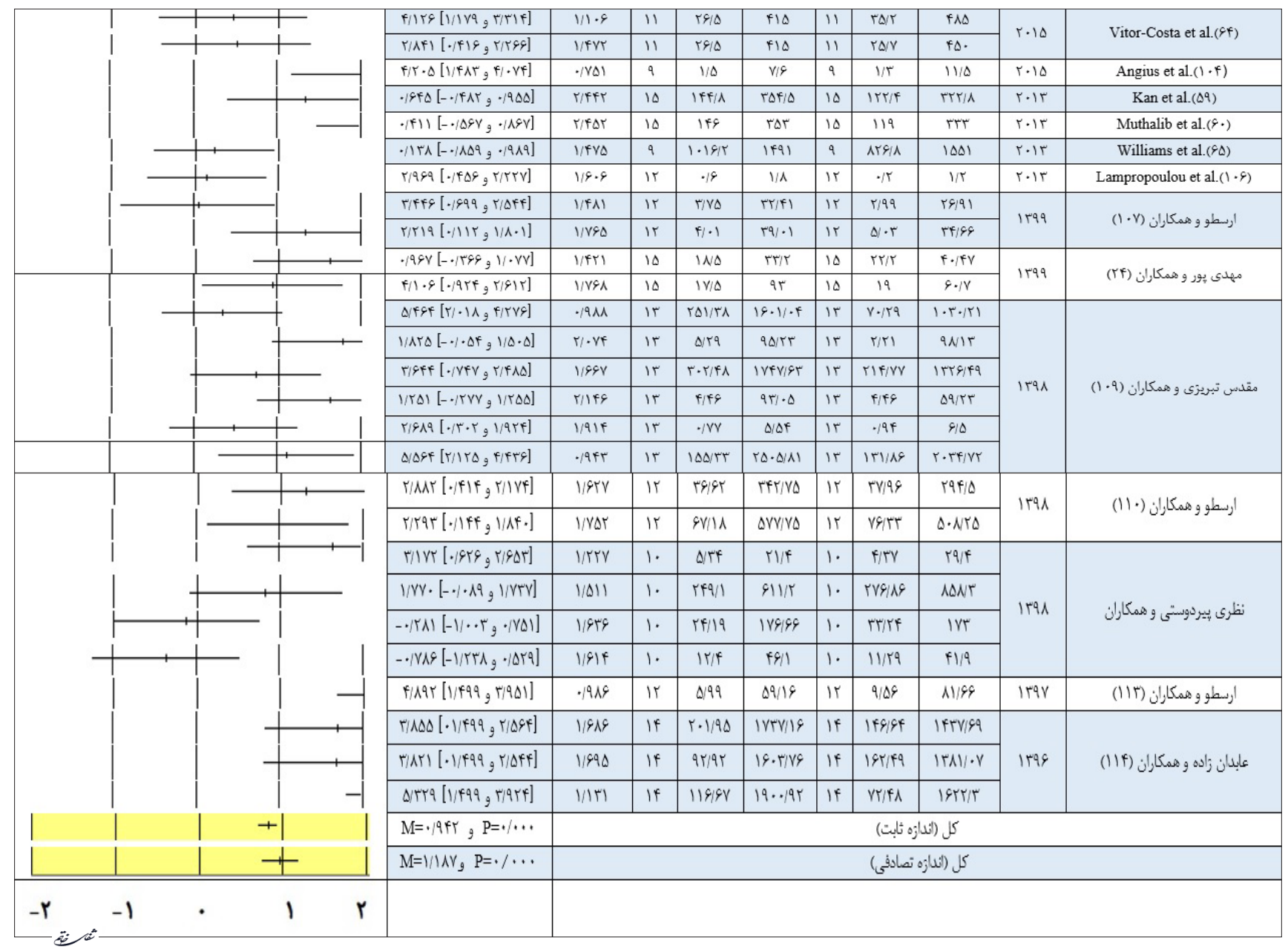

جدول بـ آمارههاى اندازئ اثر ثابت و تصادفى فراتحليل تأثير تحريك الكتريكى فراجمجمهاى بر بهينهسازى عملكرد ورزشكاران

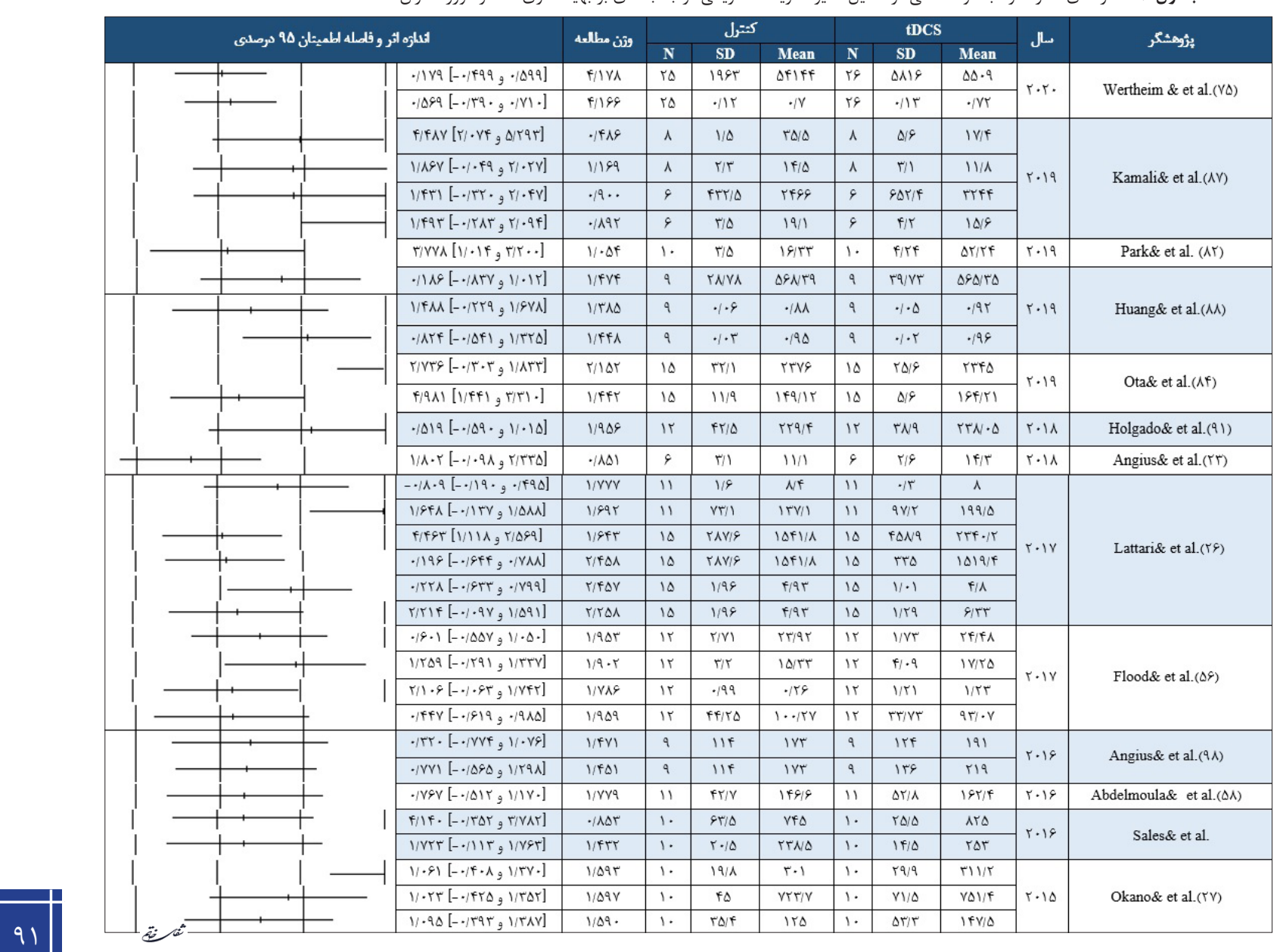




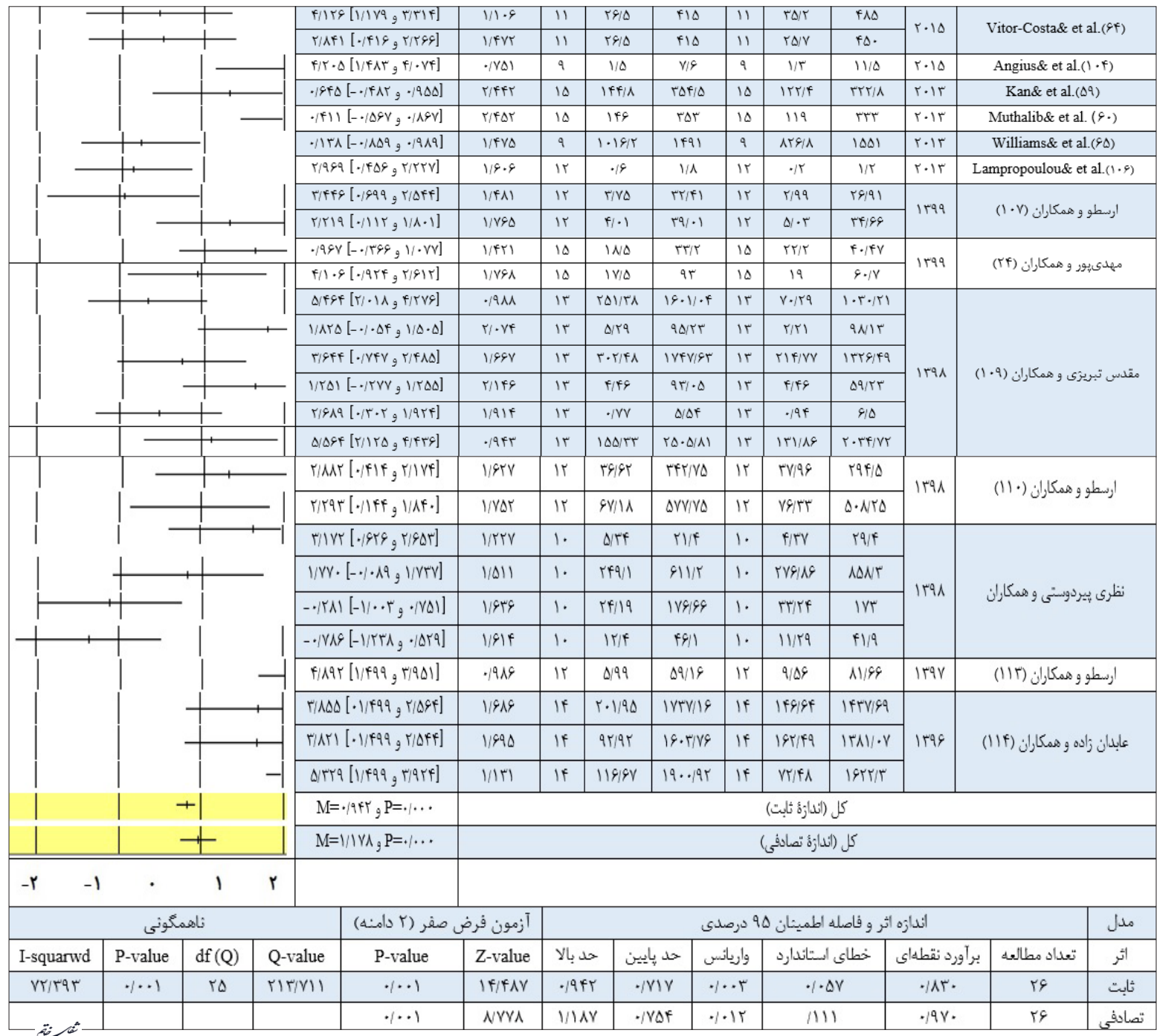

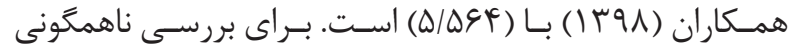

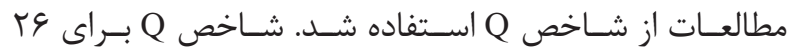

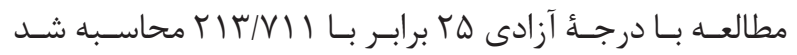

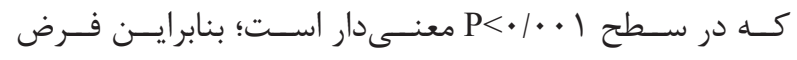

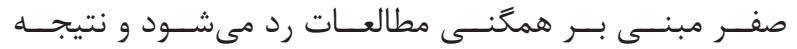

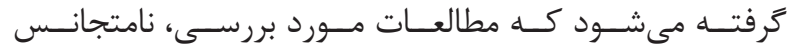

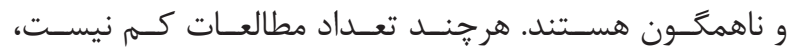

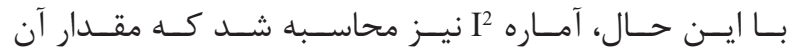

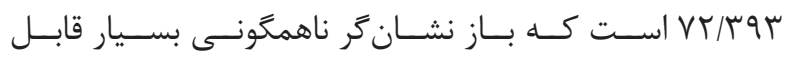

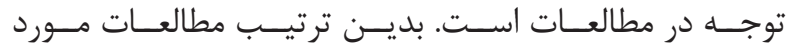

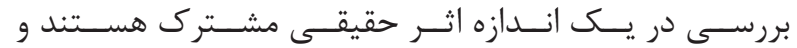

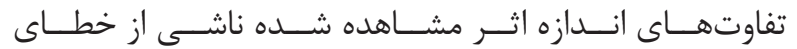

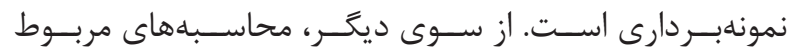

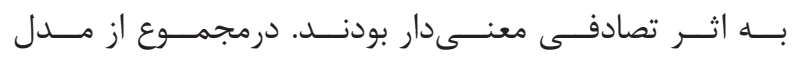

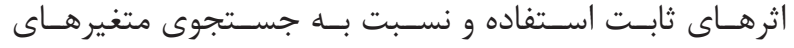

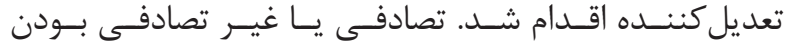

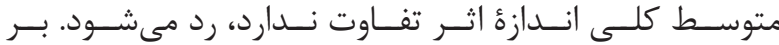

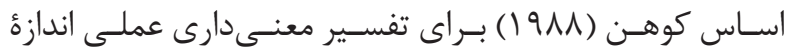

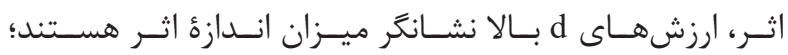

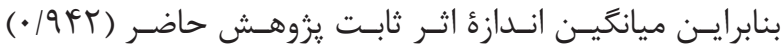

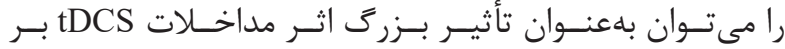

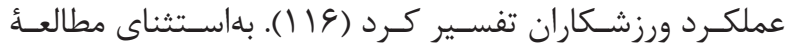
؛ $(Y \cdot 19)$.Huang $؛(Y \cdot 19)$.Kamali $؛(Y \cdot Y \cdot)$.Wertheim et al Angi- $؛(Y \cdot \mid \vee)$.Flood $(Y \cdot \mid V)$.Lattari $؛(Y \cdot \mid \Lambda)$.Holgado

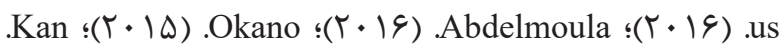

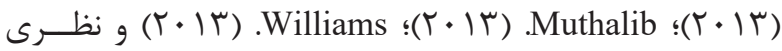

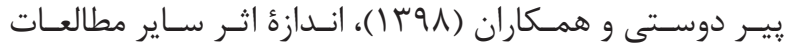

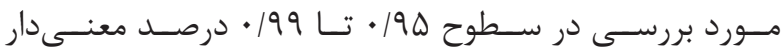

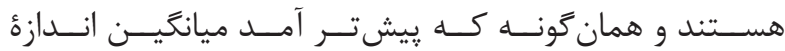

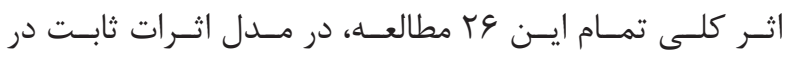

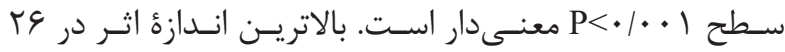

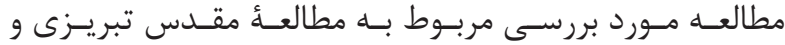




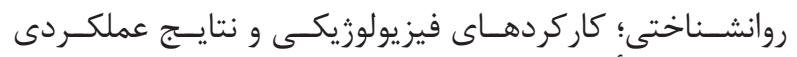

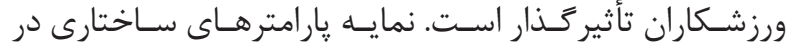

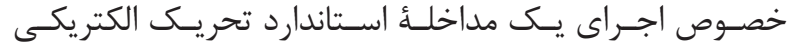

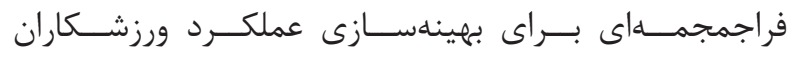

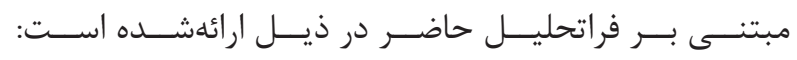

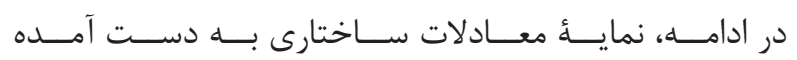

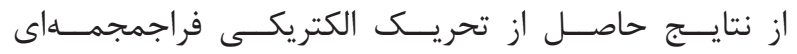

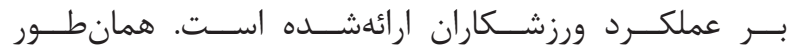

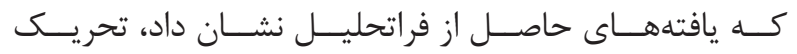

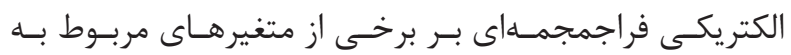

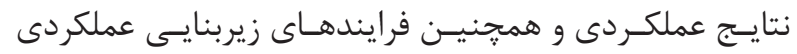

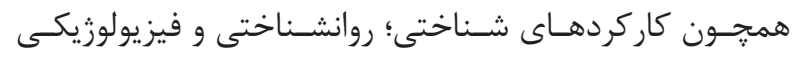

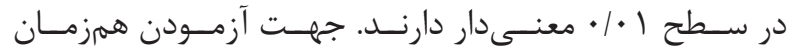

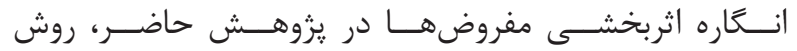

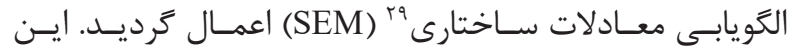

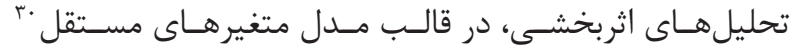

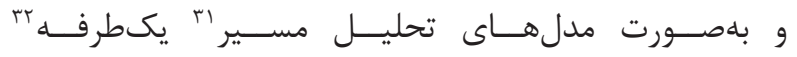

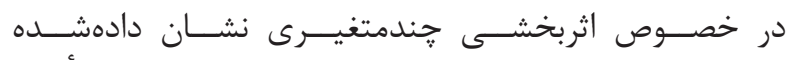

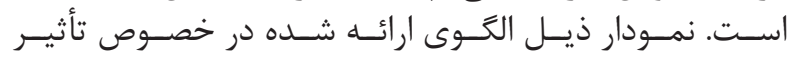

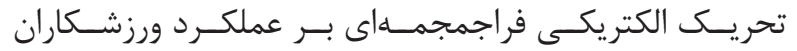

بهعنـوان متغيــر تعديل كنـــده نتايـج در نظــر كرفتــه شـد.

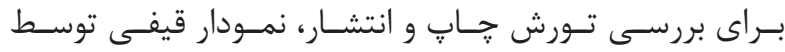

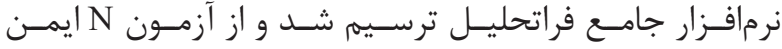

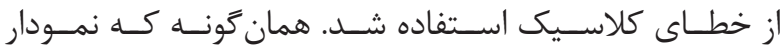

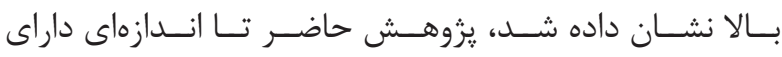

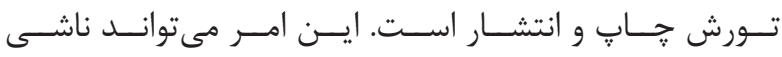

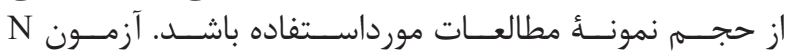

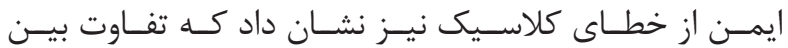

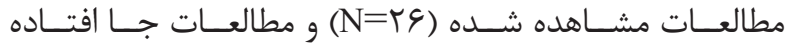
قابلتوجسه اسـت (N=FYF)

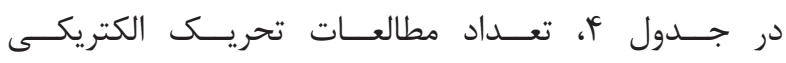

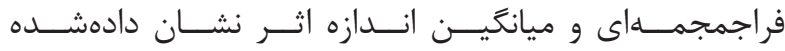

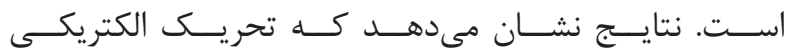

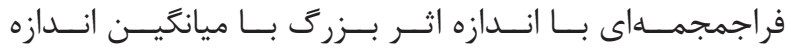

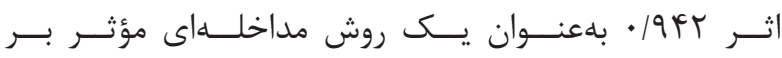

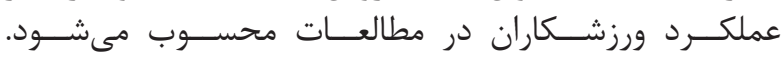

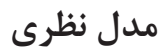

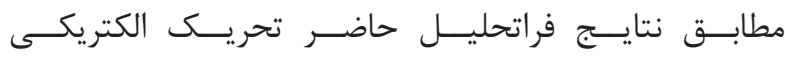

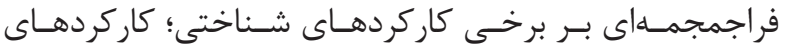

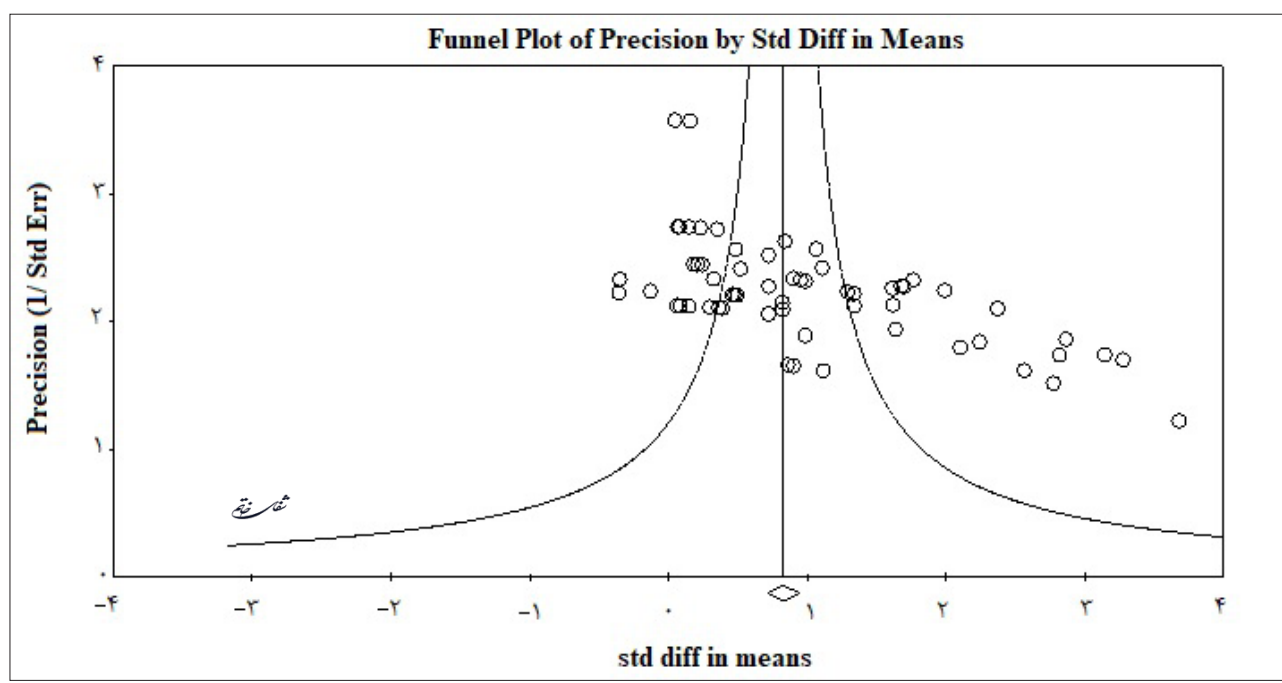

تصوير بـ - نمودار منحنى اندازٔ اثر مطالعات خطاى استاندارد ميانكَين در مدل اثرات ثابت تأثير تحريك الكتريكى فراجمجمهاى بر بهينهسازى عملكرد ورزشكاران

جدول \&- آمارههاى اندازه اثر تحريك الكتريكى فراجمجمهاى مؤثر بر بهينهسازى عملكرد ورزشكاران

\begin{tabular}{|c|c|c|c|c|}
\hline تفسير ير السامن نظار كوهن & ميالكين الثازؤ اثر & \multicolumn{2}{|c|}{ مداخلات شتاختى مؤثر ير عملكرد ورزشكاران } & تعلداد مطالعه \\
\hline 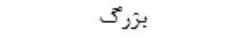 & - / 9 Ft & كل (اندازٔه ثابت) & \multirow{2}{*}{ تحريك الكتريكى فراجمجمهاى } & \multirow{2}{*}{ re } \\
\hline بزركى & W/AV & كل (اندازٔة تصادفى) & & \\
\hline
\end{tabular}

${ }^{29}$ Structural Equation Modeling (SEM)

${ }^{30}$ Independent

\section{${ }^{31}$ Path Analysis}

${ }^{32}$ Recursive 
ثم

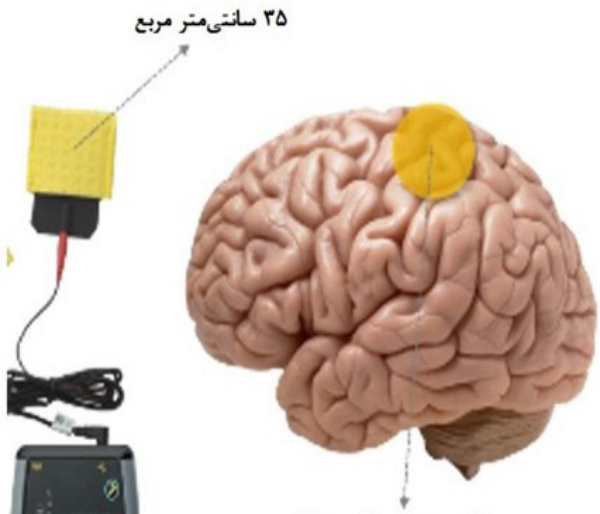

قشر حركتى اوليه (MI)

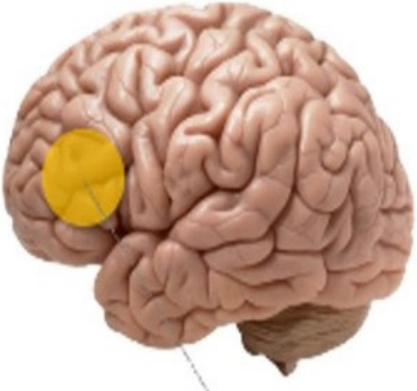

قشر جلوى بيشانى بـشتَى (DLPFC)

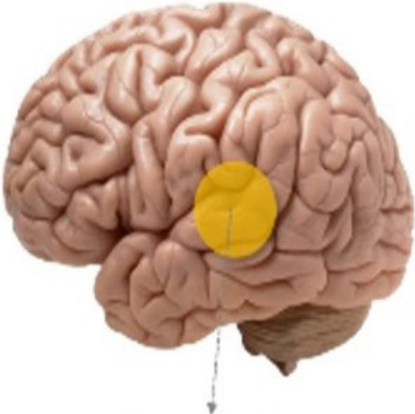

قشر اينسولار (IC)

r ميلىأمبر

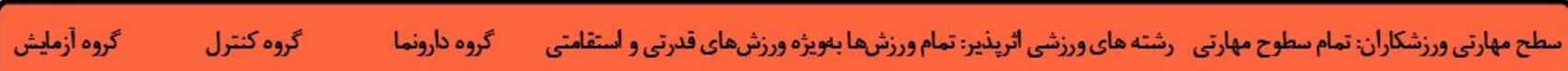

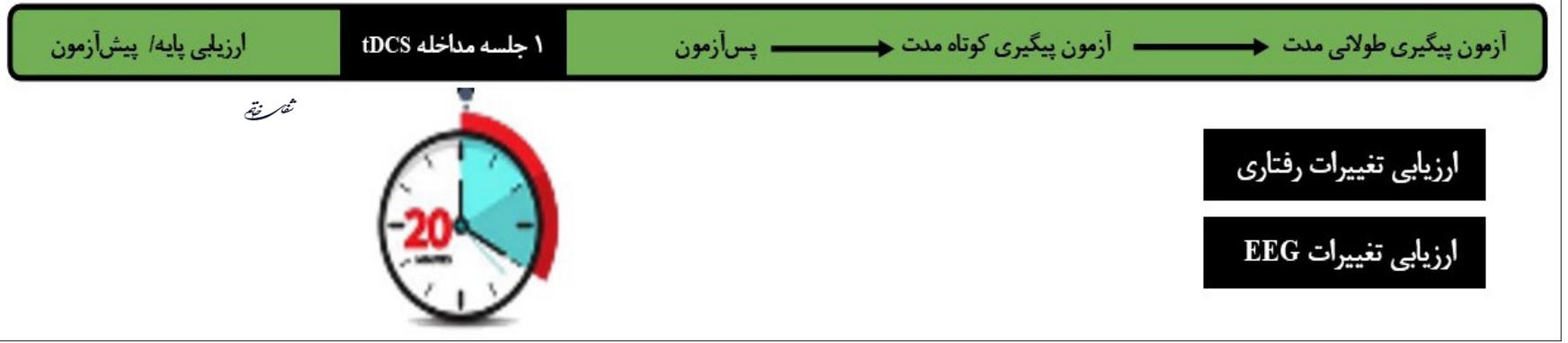

تصوير ب- نمايه يارامترهاى ساختارى در خصوص اجراى تحريك الكتريكى فراجمجمهاى براى بهينهسازى عملكرد ورزشكاران

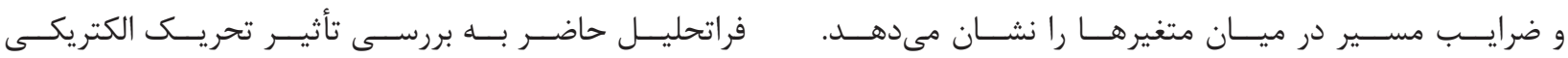

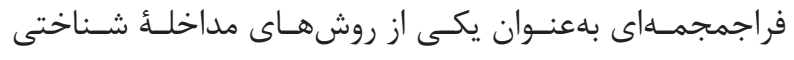

بحث و نتيجه كيرى

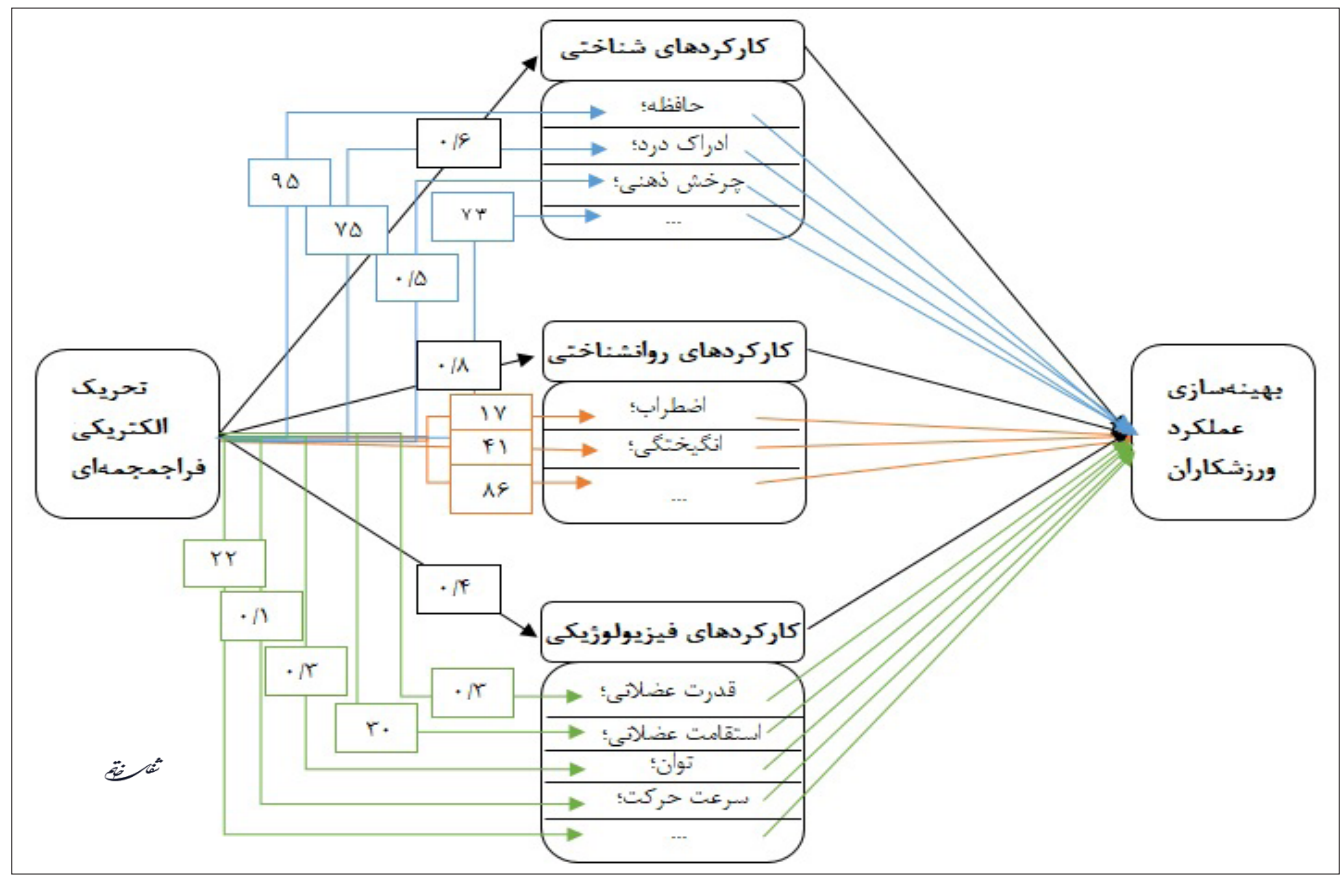

تصوير F- الكَوى تحليل مسير تأثير تحريك الكتريكى فراجمجماى بر بهينهسازى عملكرد ورزشكاران 


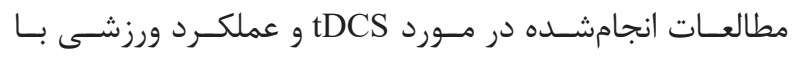

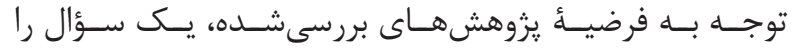

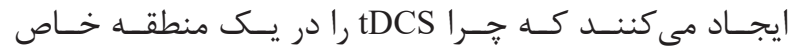

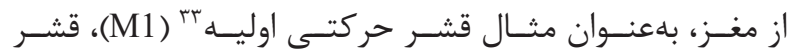

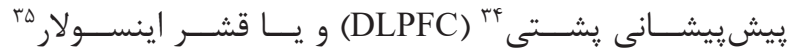

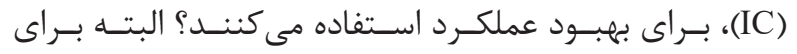

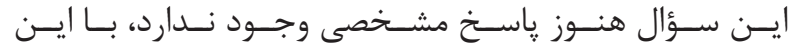

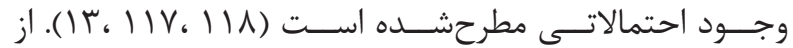

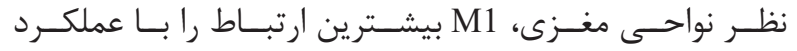

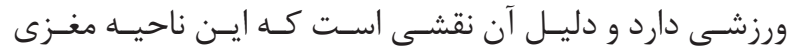

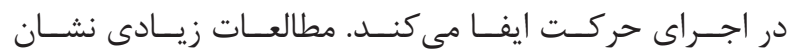

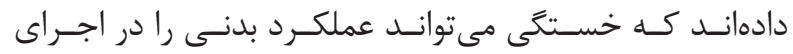

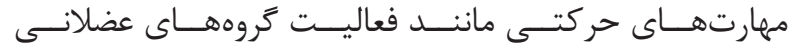

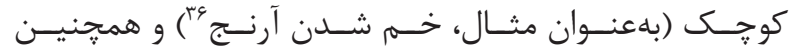

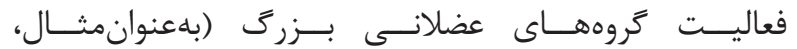

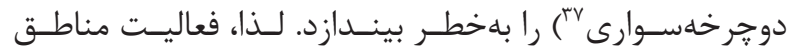

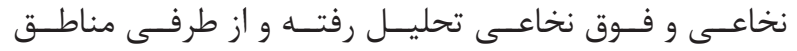

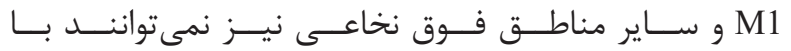

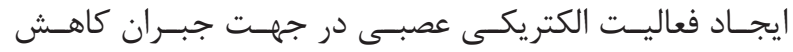

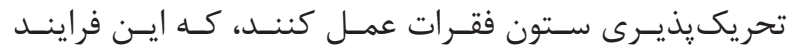

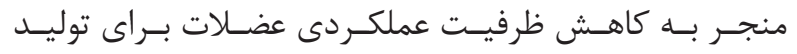

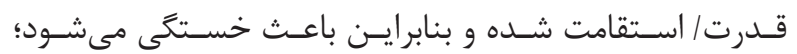

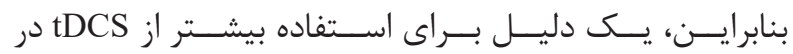

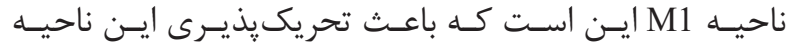

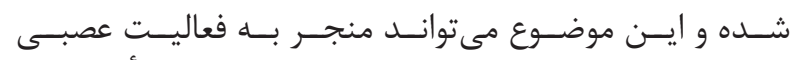

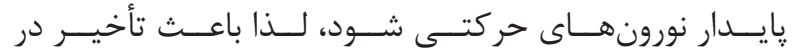

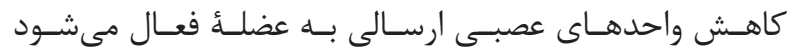

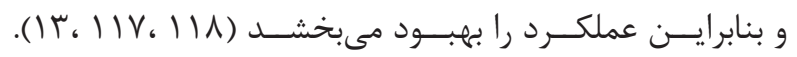

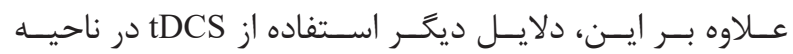

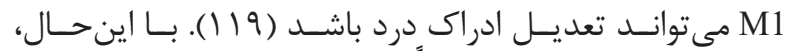

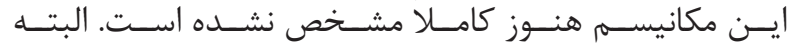

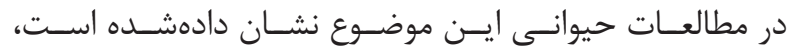

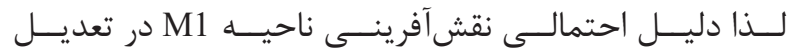

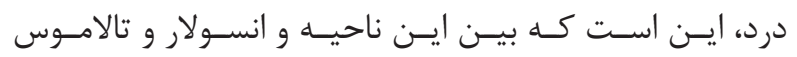

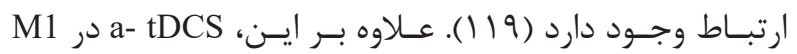

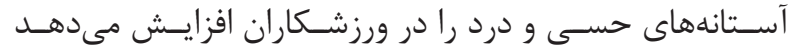

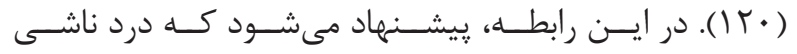

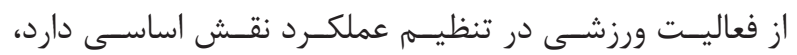

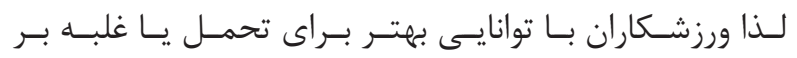

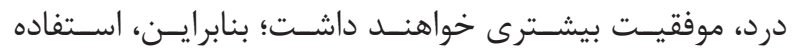

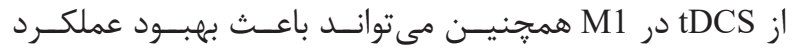

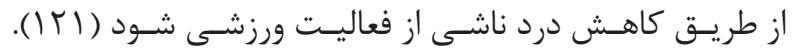

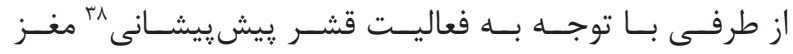

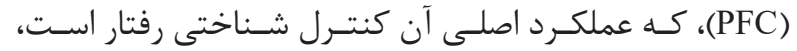

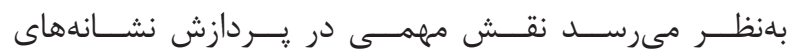

${ }^{33}$ Primary Motor Cortex (M1)

${ }^{34}$ Dorsolateral Prefrontal Cortex (DLPFC)

${ }^{35}$ Insular Cortex (IC)

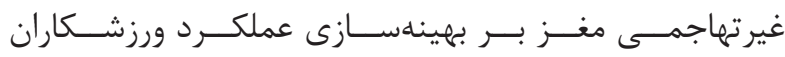

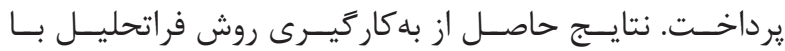

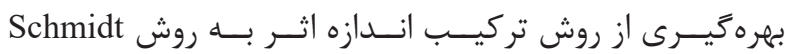
and Hunter

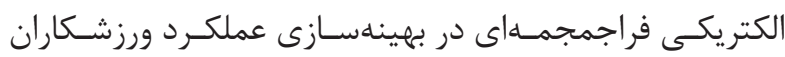

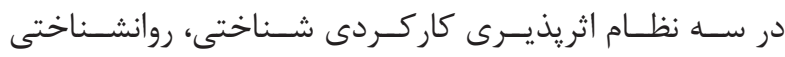

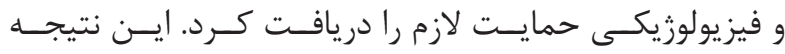

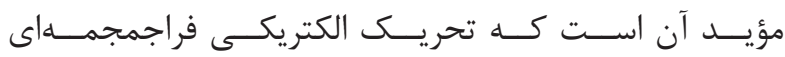

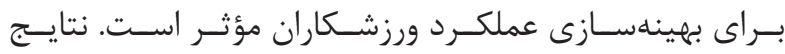

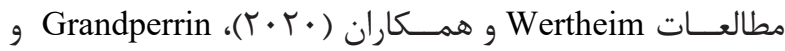

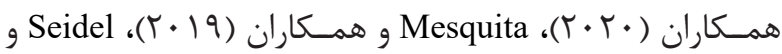

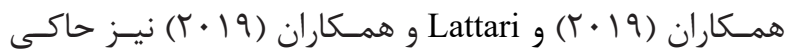

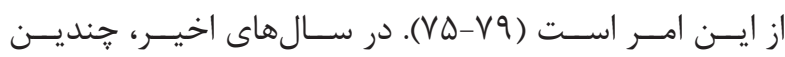

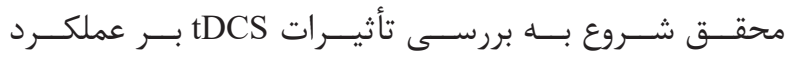

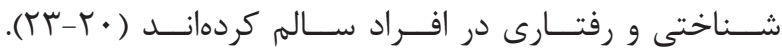

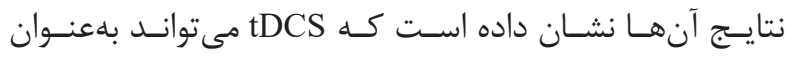

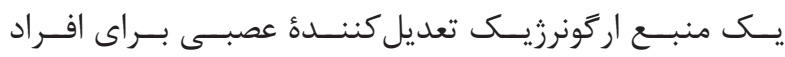

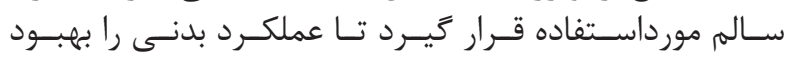

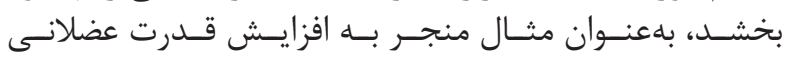

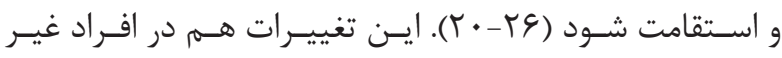

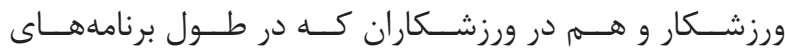

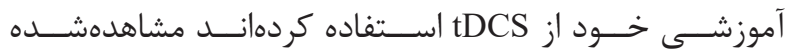

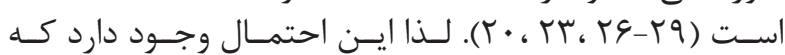

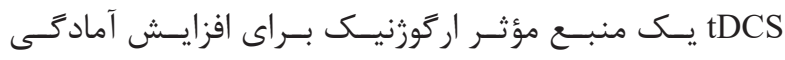

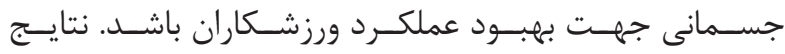

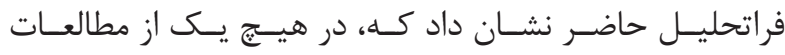

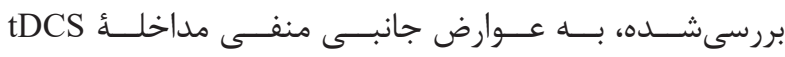

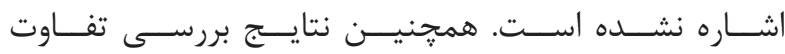

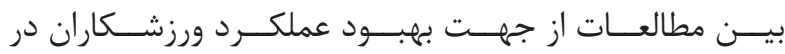

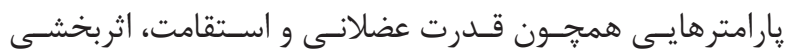

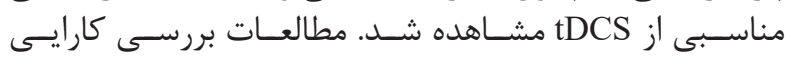

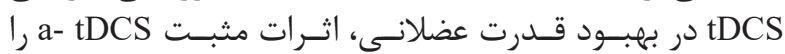

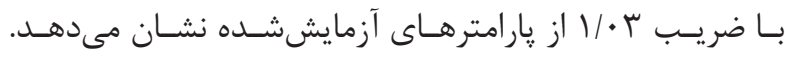

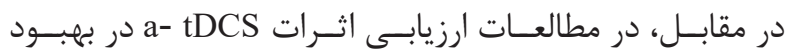

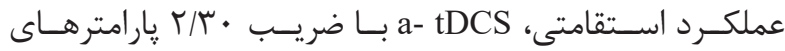

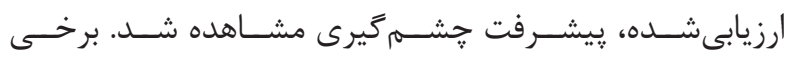

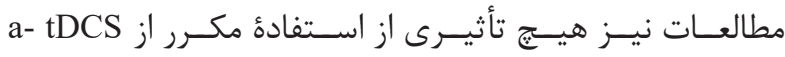

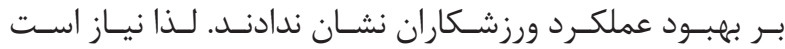

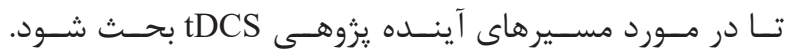

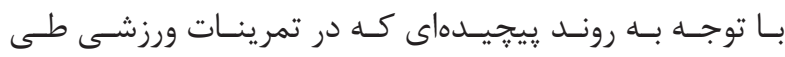

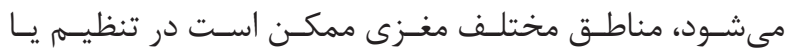

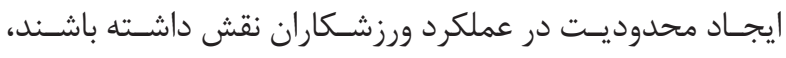

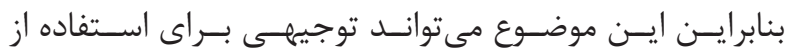

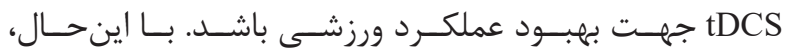

\footnotetext{
${ }^{36}$ Elbow Flexion

${ }^{37}$ Cycling

${ }^{38}$ Prefrontal Cortex
} 


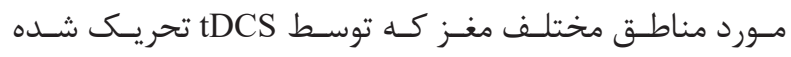

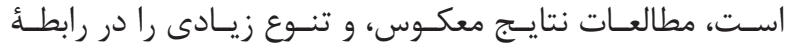

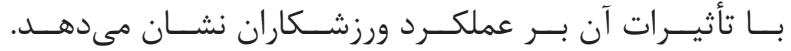

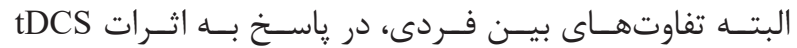

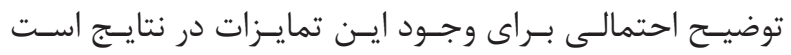

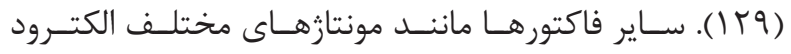

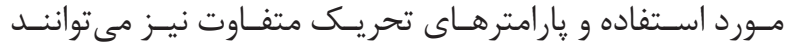

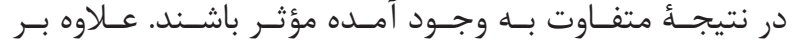

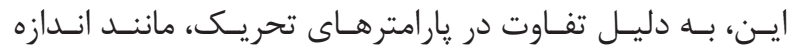

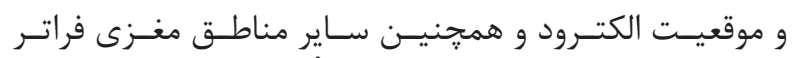

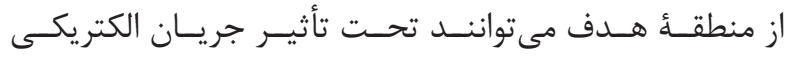

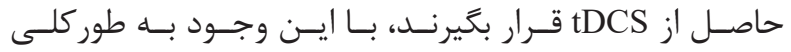

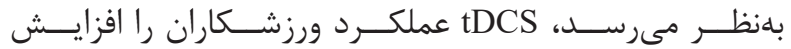

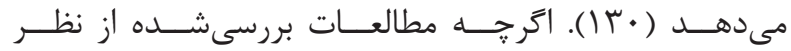

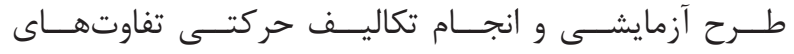

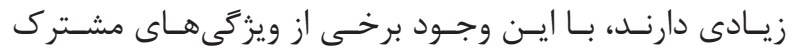

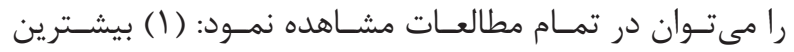

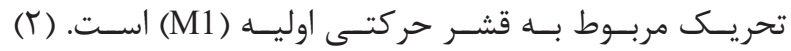

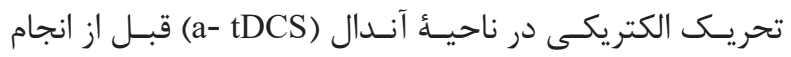

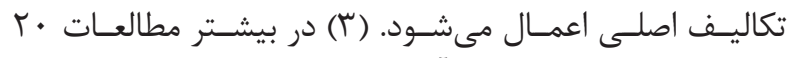

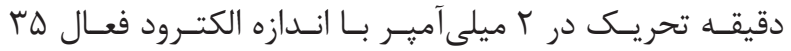

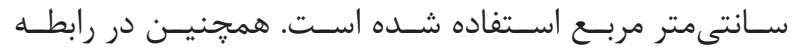

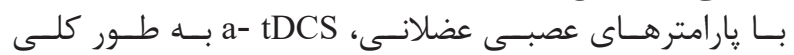

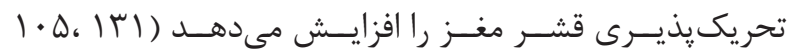

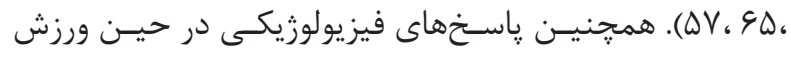

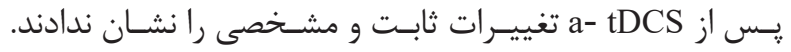

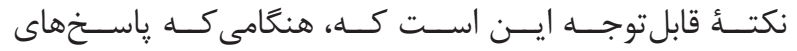

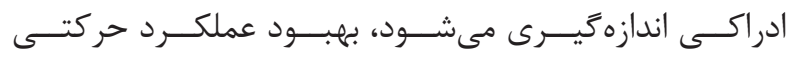

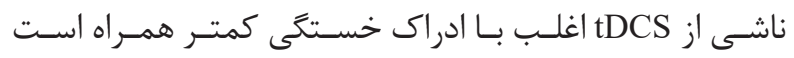

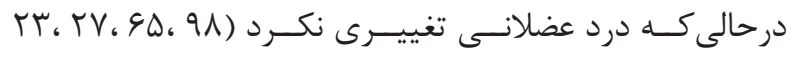

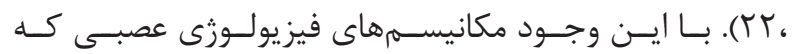

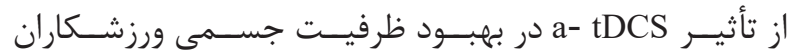

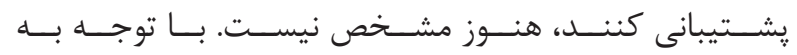

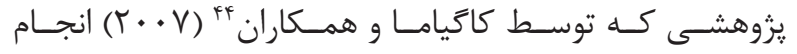

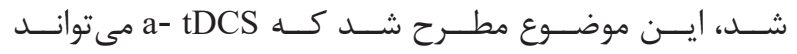

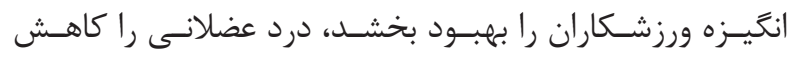

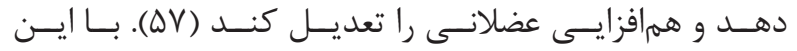

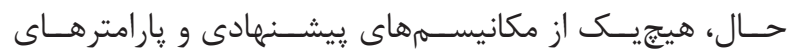

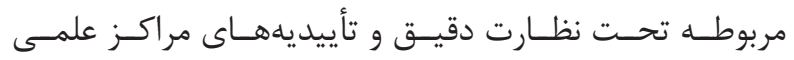

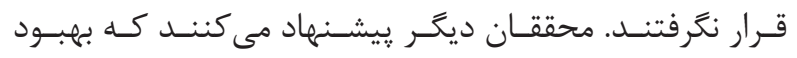

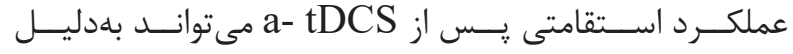

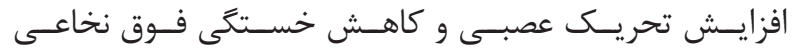

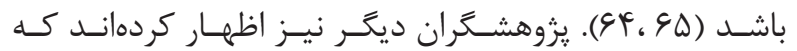
a- tDCS

\footnotetext{
${ }^{39}$ The End of The Exercise

${ }^{40}$ Effort Perception

${ }^{41}$ Oxygenation
}

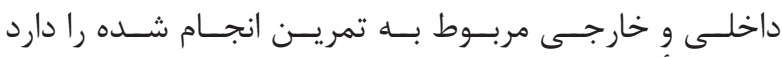

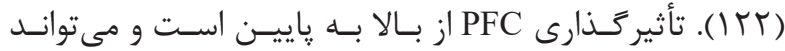

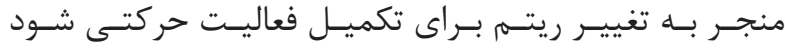

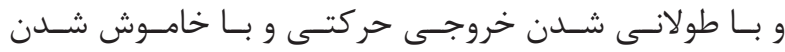

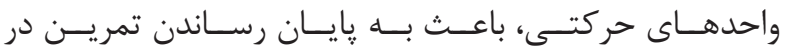

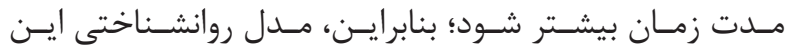

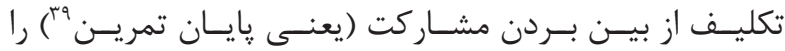

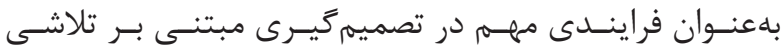

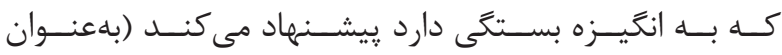

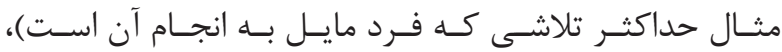

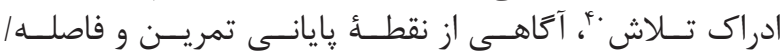

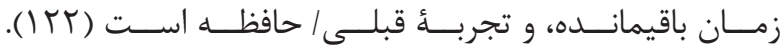

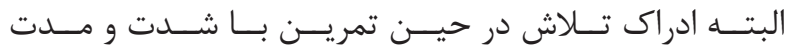

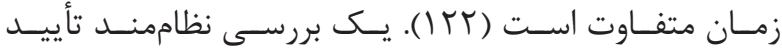

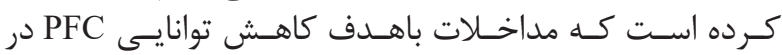

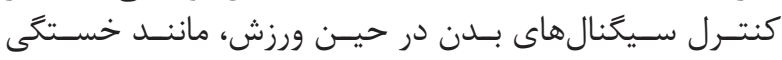

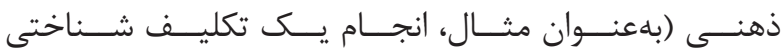

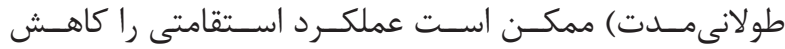

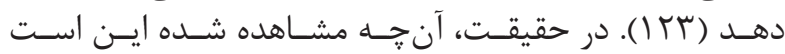

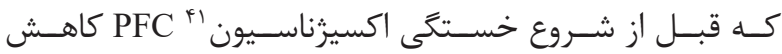

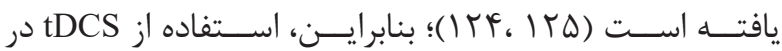

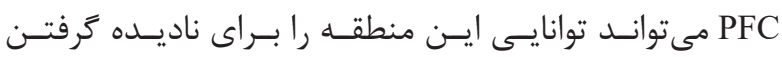

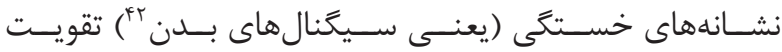

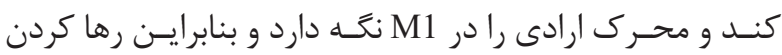

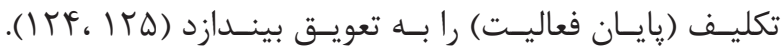

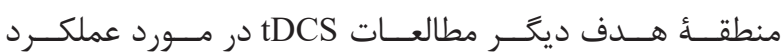

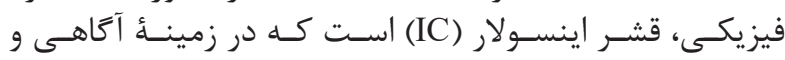

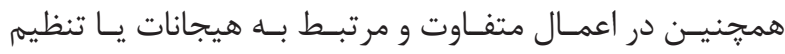

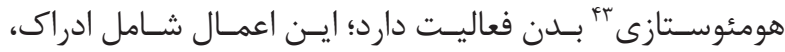

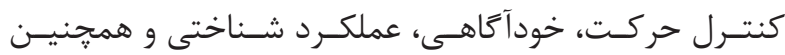

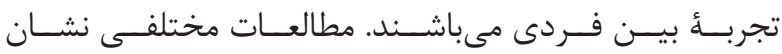

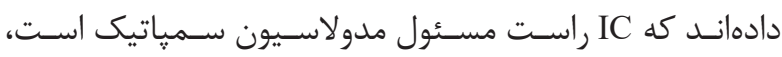

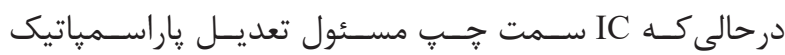

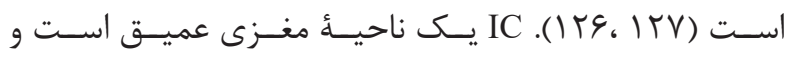

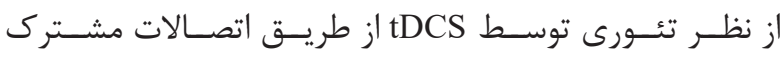

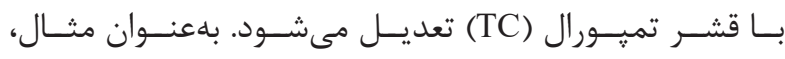

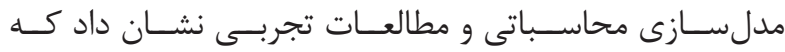

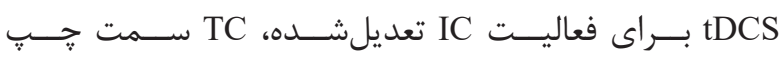

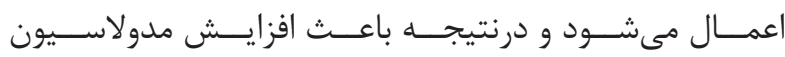

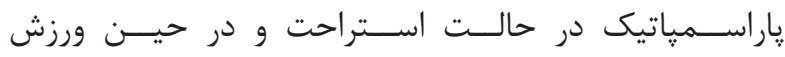

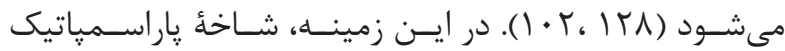

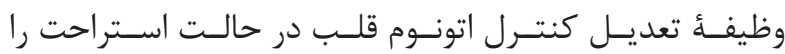

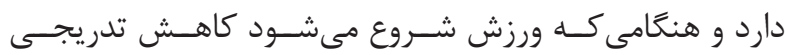

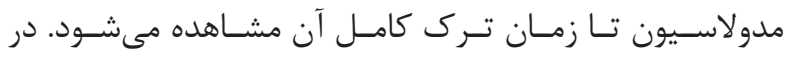

\footnotetext{
${ }^{42}$ Body Signals

${ }^{43}$ Homeostasis

${ }^{44}$ Cogiamanian
} 


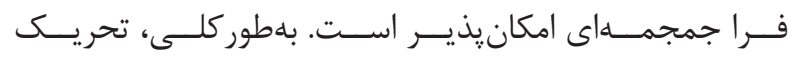

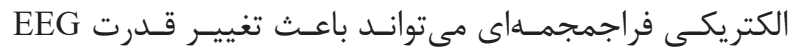

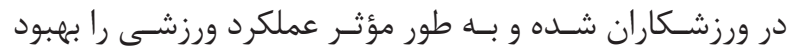

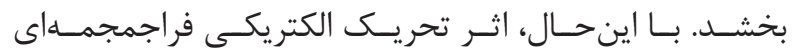

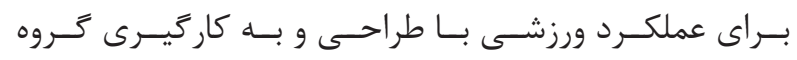

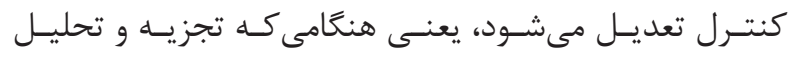

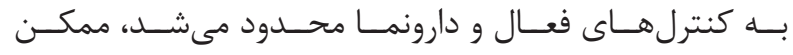

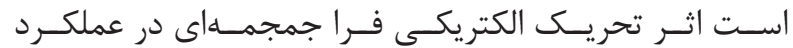

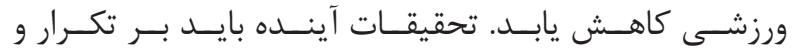

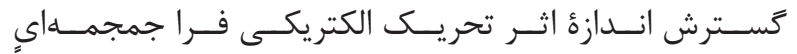

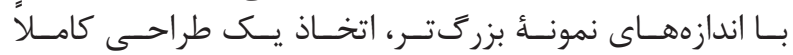

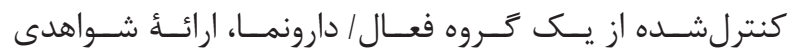

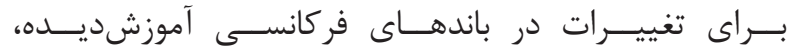

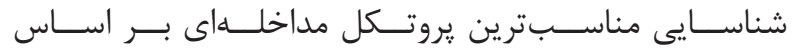

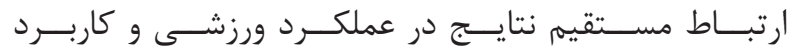

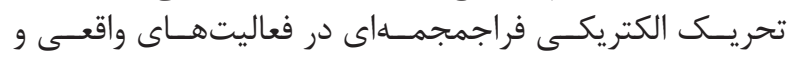

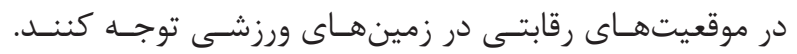

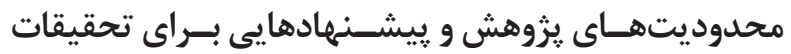

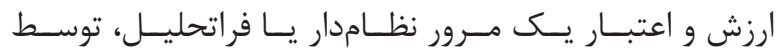

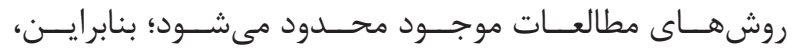

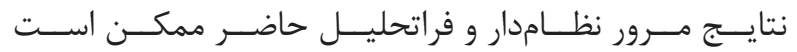

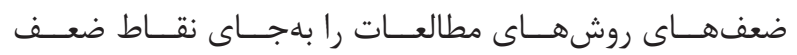

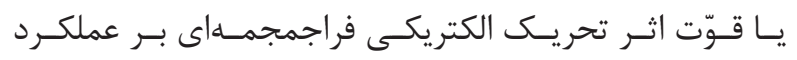

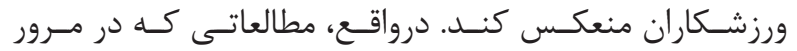

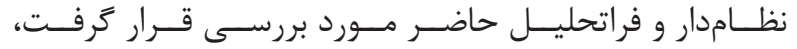

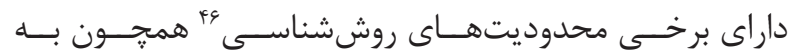

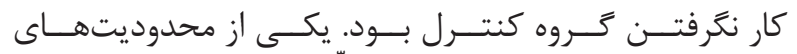

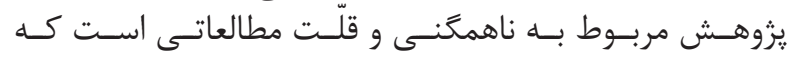

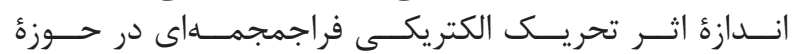

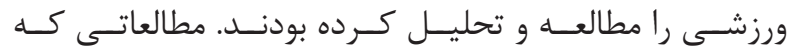

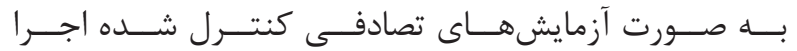

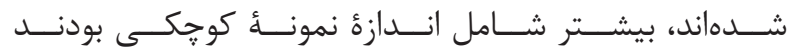

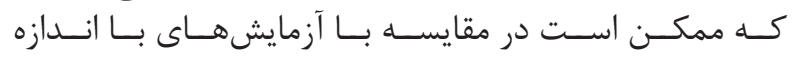

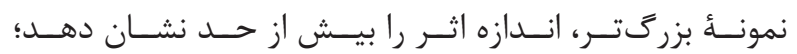

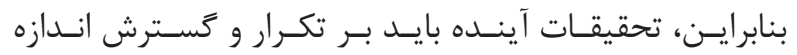

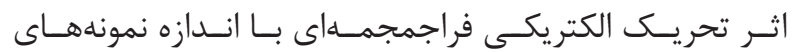

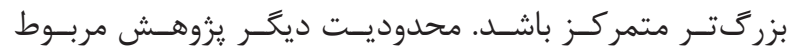

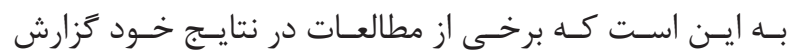

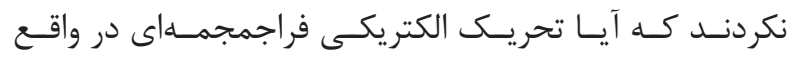

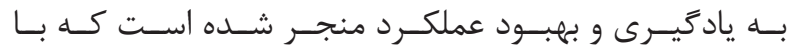

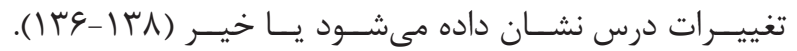

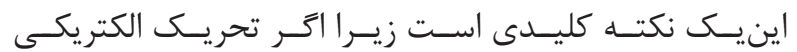

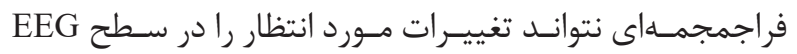

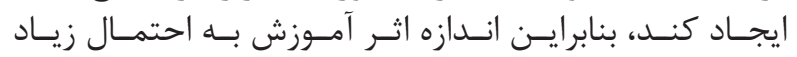

${ }^{45}$ Corticospinal

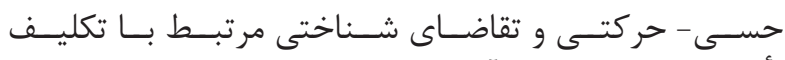

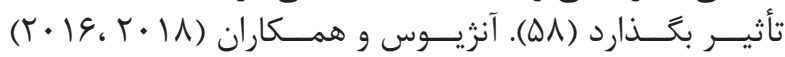

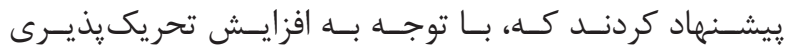

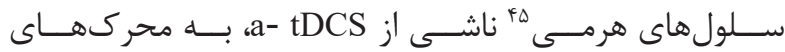

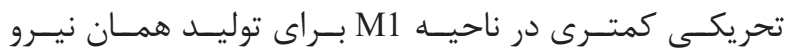

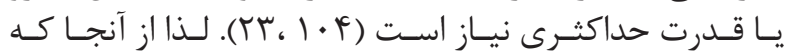

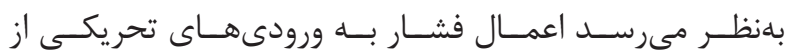

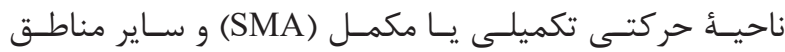

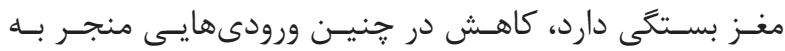

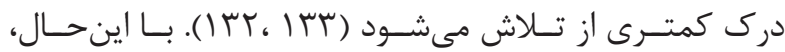

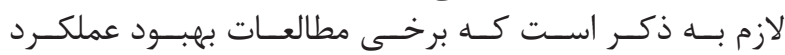

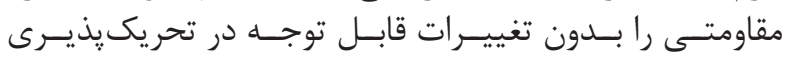

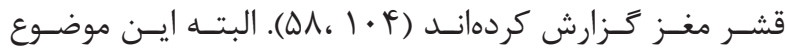

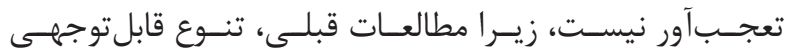

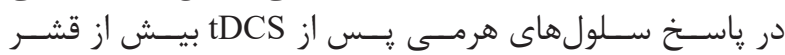

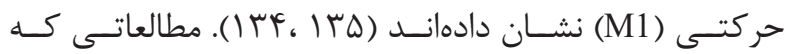

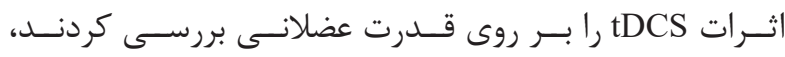

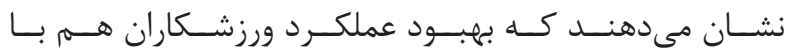

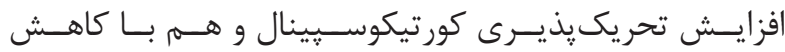

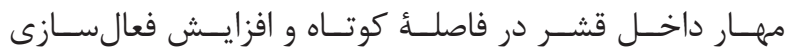

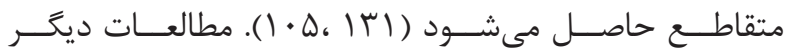

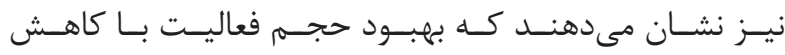

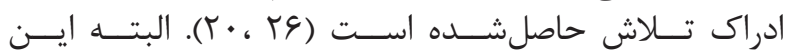

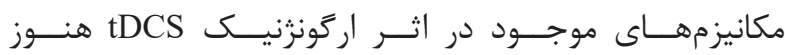

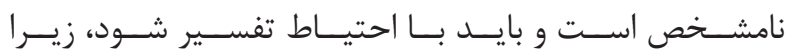

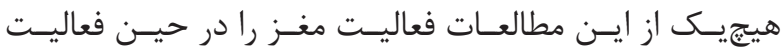

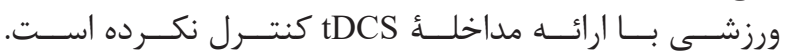

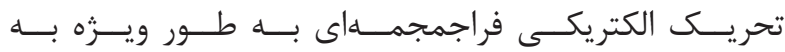

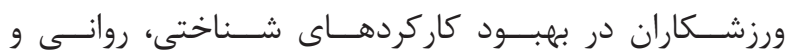

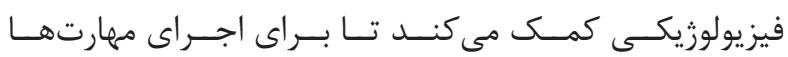

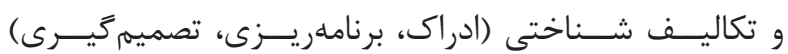

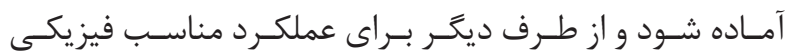

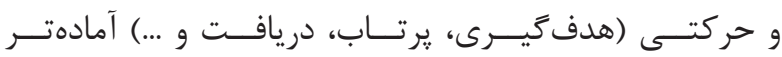

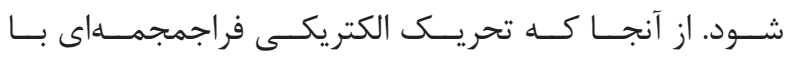

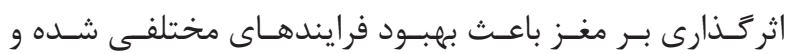

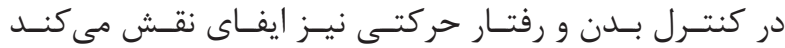

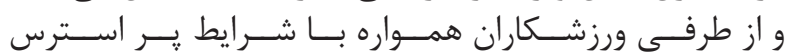

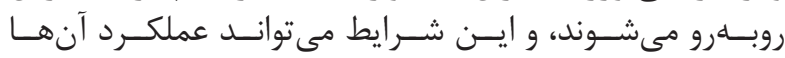

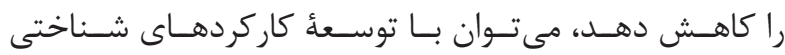

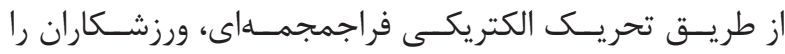

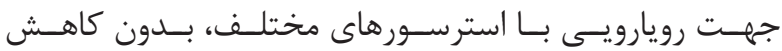

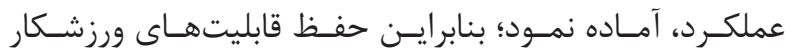

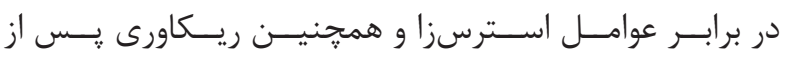

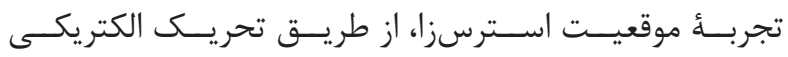

${ }^{46}$ Methodological 


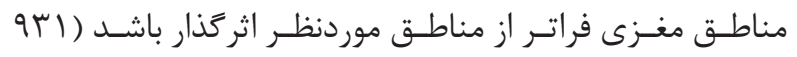

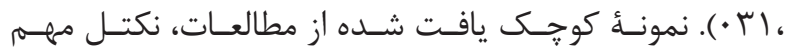

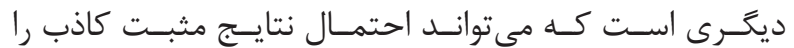

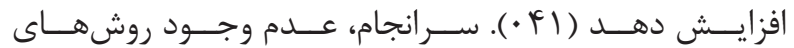

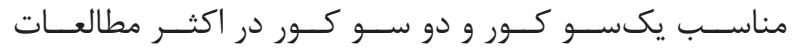

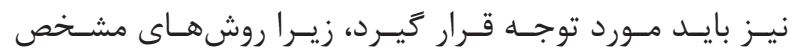

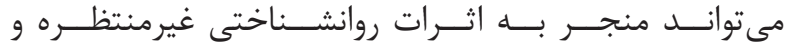

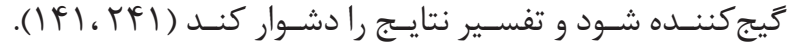
ييروى از اصول اخلاق يزوهش ني اين مقاله از نوع مرورى اسـت و نمونهُ انسـانى ياحيوانى نداشـته است.

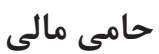

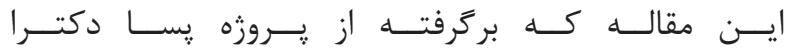

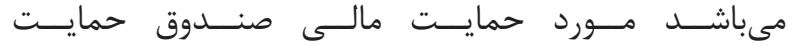

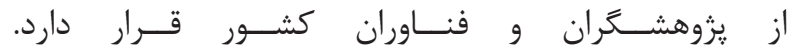
مشاركت نويسندگان

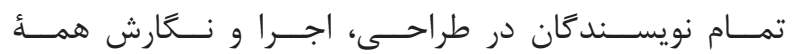

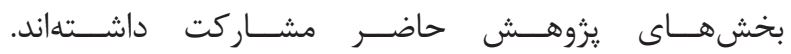

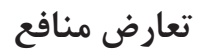

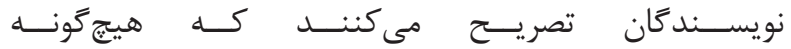

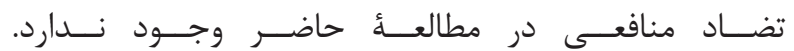

1.Bertollo M, di Fronso S, Filho E, Conforto S, Schmid M, Bortoli L, et al. Proficient brain for optimal performance: the MAP model perspective. Peer J. 2016; 4: e2082.

2.Davidson RJ, Kabat-Zinn J, Schumacher J, Rosenkranz M, Muller D, Santorelli SF, et al. Alterations in brain and immune function produced by mindfulness meditation. Psychosomatic medicine. 2003; 65(4): 564-70.

3. Ramis Y, Torregrosa M, Viladrich C, CruzJ. The Effect of Coaches Controlling Style on the Competitive Anxiety ofYoung Athletes. FrontiersinPsychology.2017; 8(572).

4. Ford JL, Ildefonso K, Jones ML, ArvinenBarrow M. Sport- related anxiety: current insights. Open Access J Sports Med. 2017; 8: 205-12.

5. Sleivert GG, Rowlands DS. Physical and physiological factors associated with success in the triathlon. Sports medicine (Auckland, NZ). 1996; 22(1): 8-18.

6. Neumayr G, Hoertnagl H, Pfister R, Koller A, Eibl G, Raas E. Physical and physiological factors associated with success in professional alpine skiing. International journal of sports medicine. 2003; 24(8): 571-5.

7. McCormick A, Meijen C, Marcora S. Psychological

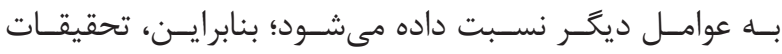

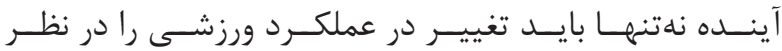

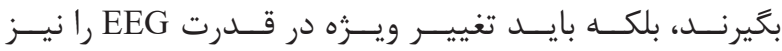

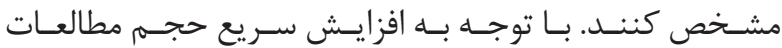

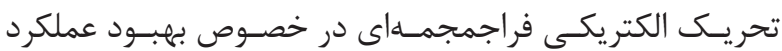

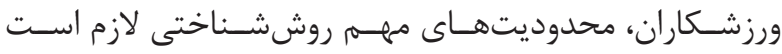

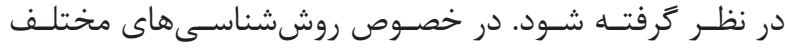

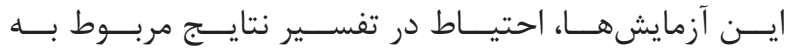

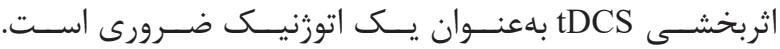

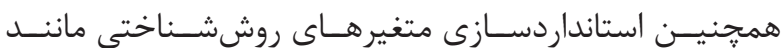

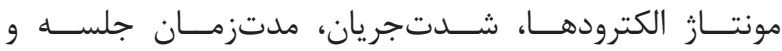

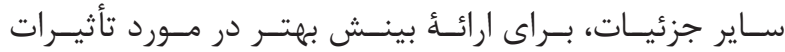

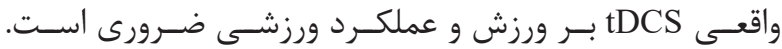

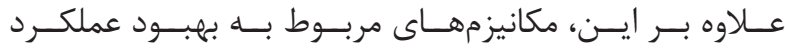

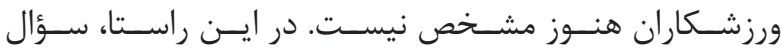

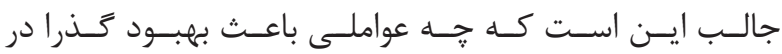

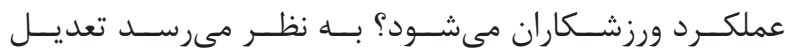

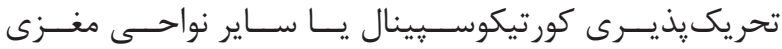

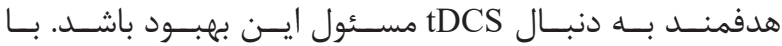

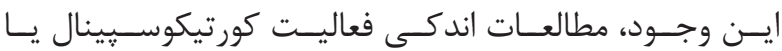

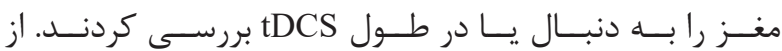

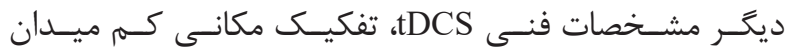

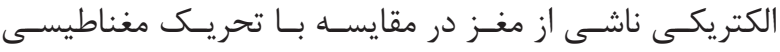

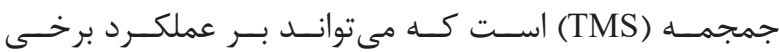

منابع

Determinants of Whole- Body Endurance Performance. Sports medicine (Auckland, NZ). 2015; 45(7): 997-1015.

8. Schubert MM, Astorino TA. A systematic review of the efficacy of ergogenic aids for improving running performance. Journal of strength and conditioning research. 2013; 27(6): 1699-707.

9. Savulescu J, Foddy B, Clayton M. Why we should allow performance enhancing drugs in sport. British journal of sports medicine. 2004; 38(6): 666-70.

10. Noakes TD. Fatigue is a Brain-Derived Emotion that Regulates the Exercise Behavior to Ensure the Protection of Whole Body Homeostasis. Frontiers in physiology. 2012; 3: 82.

11. Beck JS. Cognitive Behavior Therapy, Second Edition: Basics and Beyond. 2nd edition ed: The Guilford Press; 2011.

12. Prochaska JO, C. J. Norcross: Systems of Psychotherapy: A Transtheoretical Analysis Seventh. (7th) Edition ed: Amazon; 2009.

13. Gandevia SC. Spinal and supraspinal factors in human muscle fatigue. 
Physiological reviews. 2001; 81(4): 1725-89.

14. Noakes TD. Time to move beyond a brainless exercise physiology: the evidence for complex regulation of human exercise performance. Applied physiology, nutrition, and metabolism $=$ Physiologie appliquee, nutrition et metabolisme. 2011; 36(1): 23-35.

15.AngiusL,HopkerJ,MaugerAR. TheErgogenicEffects of Transcranial Direct Current Stimulation on Exercise Performance. Frontiers in physiology. 2017; 8: 90.

16. Nitsche MA, Paulus W. Excitability changes induced in the human motor cortex by weak transcranial direct current stimulation. The Journal of physiology. 2000; $527 \mathrm{Pt} 3(\mathrm{Pt} 3)$ : 633-9.

17. Stagg CJ, Nitsche MA. Physiological basis of transcranial direct current stimulation. The Neuroscientist: a review journal bringing neurobiology, neurology and psychiatry. 2011; 17(1): 37-53.

18. Bikson M, Inoue M, Akiyama H, Deans JK, Fox JE, Miyakawa $\mathrm{H}$, et al. Effects of uniform extracellular DC electric fields on excitability in rat hippocampal slices in vitro. The Journal of physiology. 2004; 557(Pt 1): 175-90.

19. Rahman A, Reato D, Arlotti M, Gasca F, Datta A, Parra LC, et al. Cellular effects of acute direct current stimulation: somatic and synaptic terminal effects. The Journal of physiology. 2013; 591(10): 2563-78.

20. Lattari E, Andrade ML, Filho AS, Moura AM, Neto GM, Silva JG, et al. Can Transcranial Direct Current Stimulation Improve the Resistance Strength and Decrease the Rating Perceived Scale in Recreational Weight-Training Experience? Journal of strength and conditioning research. 2016; 30(12): 3381-7.

21. Lattari E, Campos C, Lamego MK, Legey S, Neto GM, Rocha NB, et al. Can Transcranial Direct Current Stimulation Improve Muscle Power in Individuals With Advanced Weight-Training Experience? Journal of strength and conditioning research. 2020; 34(1): 97-103.

22. Lattari E, Rosa Filho BJ, Fonseca Junior SJ, MurilloRodriguez E, Rocha N, Machado S, et al. Effects on Volume Load and Ratings of Perceived Exertion in Individuals' Advanced Weight Training After Transcranial Direct Current Stimulation. Journal of strength and conditioning research. 2020; 34(1): 89-96.

23. Angius L, Mauger AR, Hopker J, PascualLeone A, Santarnecchi E, Marcora SM. Bilateral extracephalic transcranial direct current stimulation improves endurance performance in healthy individuals. Brain stimulation. 2018; 11(1): 108-17.

24. Roya M, Mahdi N, Rokhsareh B, Hamid M.
Comparison of effect of the transcranial direct current stimulation (tDCS) of vision and motor cortex on learning of basketball free throw. Development \& Motor Learning. 2020; 12(40): 153-68.

25. Golandam Z, Mohammad Reza D. The effect of Transcranial Direct Current Stimulation on Working Memory and Reaction time in Athlete Girls. Journal of Neuropsychology. 2017; 3(10): 51-62.

26. Lattari E, de Oliveira BS, Oliveira BRR, de Mello Pedreiro RC, Machado S, Neto GAM. Effects of transcranial direct current stimulation on time limit and ratings of perceived exertion in physically active women. Neuroscience letters. 2018; 662: 12-6.

27. Okano AH, Fontes EB, Montenegro RA, Farinatti Pde T, Cyrino ES, Li LM, et al. Brain stimulation modulates the autonomic nervous system, rating of perceived exertion and performance during maximal exercise. British journal of sports medicine. 2015; 49(18): 1213-8.

28. Reardon S. 'Brain doping may improve athlete's performance. Nature. 2016; 531(7594): 283-4.

29. Edwards DJ, Cortes M, Wortman- Jutt S, Putrino D, Bikson M, Thickbroom G, et al. Transcranial Direct Current Stimulation and Sports Performance. Frontiers in human neuroscience. 2017; 11: 243.

30. Janssens AC, Kraft P. Research conducted using data obtained through online communities: ethical implications of methodological limitations. PLoS medicine. 2012; 9(10): e1001328.

31. Bain L., Norris S., C. S. Non-Invasive Neuromodulation of the Central Nervous System: Opportunities and Challenges: Workshop Summary. Washington (DC): National Academies Press (US).

32. Fregni F, Nitsche MA, Loo CK, Brunoni AR, Marangolo P, Leite J, et al. Regulatory Considerations for the Clinical and Research Use of Transcranial Direct Current Stimulation (tDCS): review and recommendations from an expert panel. Clinical research and regulatory affairs. 2015; 32(1): 22-35.

33. Bikson M, Grossman P, Thomas C, Zannou AL, Jiang J, Adnan T, et al. Safety of Transcranial Direct Current Stimulation: Evidence Based Update 2016. Brain stimulation. 2016; 9(5): 641-61.

34. Kuersten A, Hamilton RH. Minding the 'gaps' in the federal regulation of transcranial direct current stimulation devices. Journal of Law and the Biosciences. 2016; 3(2):309-17.

35. Zettler PJ. What lies ahead for FDA regulation of tDCS products? Journal of Law 


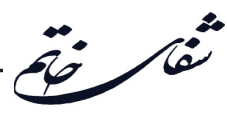

and the Biosciences. 2016; 3(2): 318-23.

36. Datta A, Bansal V, Diaz J, Patel J, Reato D, Bikson M. Gyri-precise head model of transcranial direct current stimulation: improved spatial focality using a ring electrode versus conventional rectangular pad. Brain stimulation. 2009; 2(4): 201-7.

37. Luu P, Essaki Arumugam EM, Anderson E, Gunn A, Rech D, Turovets S, et al. Slow-Frequency Pulsed Transcranial Electrical Stimulation for Modulation of Cortical Plasticity Based on Reciprocity Targeting with Precision Electrical Head Modeling. Frontiers in human neuroscience. 2016; 10: 377.

38. Baudewig J, Nitsche MA, Paulus W, Frahm J. Regional modulation of BOLD MRI responses to human sensorimotor activation by transcranial direct current stimulation. Magnetic resonance in medicine. 2001; 45(2): 196-201.

39. DosSantos MF, Love TM, Martikainen IK, Nascimento TD, Fregni F, Cummiford C, et al. Immediate effects of tDCS on the $\mu$-opioid system of a chronic pain patient. Frontiers in psychiatry. 2012; 3: 93.

40. Jog MV, Smith RX, Jann K, Dunn W, Lafon B, Truong $\mathrm{D}$, et al. In-vivo Imaging of Magnetic Fields Induced by TranscranialDirectCurrentStimulation(tDCS)inHuman Brain using MRI. Scientific Reports. 2016; 6(1): 34385.

41. Huang Y, Liu AA, Lafon B, Friedman D, Dayan $M$, Wang $X$, et al. Measurements and models of electric fields in the in vivo human brain during transcranial electric stimulation. 2017;6.

42. Edwards D, Cortes M, Datta A, Minhas P, Wassermann EM, Bikson M. Physiological and modeling evidence for focal transcranial electrical brain stimulation in humans: a basis for highdefinition tDCS. NeuroImage. 2013; 74: 266-75.

43. Strube W, Bunse T, Nitsche MA, Nikolaeva A, Palm U, Padberg F, et al. Bidirectional variability in motor cortex excitability modulation following 1 $\mathrm{mA}$ transcranial direct current stimulation in healthy participants. Physiological reports. 2016;15)4 ).

44. Hamner JW, Villamar MF, Fregni F, Taylor JA. Transcranial direct current stimulation (tDCS) and the cardiovascular responses to acute pain in humans. Clinical neurophysiology: official journal of the International Federation of Clinical Neurophysiology. 2015; 126(5): 1039-46.

45. Wagner S, Lucka F, Vorwerk J, Herrmann CS, Nolte G, Burger $M$, et al. Using reciprocity for relating the simulation of transcranial current stimulation to the EEG forward problem. NeuroImage. 2016; 140: 163-73.
46. Woods AJ, Antal A, Bikson M, Boggio PS, Brunoni AR, Celnik P, et al. A technical guide to tDCS, and related non- invasive brain stimulation tools. Clinical neurophysiology: official journal of the International Federation of Clinical Neurophysiology. 2016; 127(2): 1031-48.

47. Wexler A. The practices of do- it- yourself brain stimulation: implications for ethical considerations and regulatory proposals. Journal of medical ethics. 2016; 42(4): 211-5.

48. Wurzman R, Hamilton RH, Pascual- Leone A, Fox MD. An open letter concerning do- ityourself users of transcranial direct current stimulation. Annals of neurology. 2016; 80(1): 1-4.

49. Crewther BT, Carruthers J, Kilduff LP, Sanctuary CE, Cook CJ. Temporal associations between individual changes in hormones, training motivation and physical performance in elite and non- elite trained men. Biology of sport. 2016; 33(3): 215-21.

50. Rich TA, Pfister R, Alton J, Gerdt D, Baruch M. Assessment of Cardiovascular Parameters during Meditation with Mental Targeting in Varsity Swimmers. Evidence- Based Complementary and Alternative Medicine. 2016; 2016: 7923234.

51. Ahmedov S. Ergogenic effect of acupuncture in sport and exercise: a brief review. Journal of strength and conditioning research. 2010; 24(5): 1421-7.

52. Zhu B, Wang Y, Zhang G, Ouyang H, Zhang J, Zheng $\mathrm{Y}$, et al. Acupuncture at $\mathrm{KI} 3$ in healthy volunteers induces specific cortical functional activity: an fMRI study. BMC complementary and alternative medicine. 2015; 15: 361.

53.JarrayaM, ChtourouH,AlouiA,HammoudaO, Chamari $\mathrm{K}$, Chaouachi A, et al. The Effects of Music on Highintensity Short- term Exercise in Well Trained Athletes. Asian journal of sports medicine. 2012; 3(4): 233-8.

54. Sabino- Carvalho JL, Lopes TR, Obeid- Freitas T, Ferreira TN, Succi JE, Silva AC, et al. Effect of Ischemic Preconditioning on Endurance Performance Does Not Surpass Placebo. Medicine and science in sports and exercise. 2017; 49(1): 124-32.

55. Clark VP, Coffman BA, Mayer AR, Weisend MP, Lane TD, Calhoun VD, et al. TDCS guided using fMRI significantly accelerates learning to identify concealed objects. NeuroImage. 2012; 59(1): 117-28.

56. Flood A, Waddington G, Keegan RJ, Thompson KG, Cathcart S. The effects of elevated pain inhibition on endurance exercise performance. PeerJ. 2017; 5: e3028.

57. Cogiamanian F, Marceglia S, Ardolino G, Barbieri 
S, Priori A. Improved isometric force endurance after transcranial direct current stimulation over the human motor cortical areas. The European journal of neuroscience. 2007; 26(1): 242-9.

58. Abdelmoula A, Baudry S, Duchateau J. Anodal transcranial direct current stimulation enhances time to task failure of a submaximal contraction of elbow flexors without changing corticospinal excitability. Neuroscience. 2016; 322: 94-103.

59. Kan B, Dundas JE, Nosaka K. Effect of transcranial direct current stimulation on elbow flexor maximal voluntary isometric strength and endurance. Applied physiology, nutrition, and metabolism Physiologie appliquee, nutrition et metabolisme. 2013; 38(7): 734-9.

60. Muthalib M, Kan B, Nosaka K, Perrey S. Effects of transcranial direct current stimulation of the motor cortex on prefrontal cortex activation during a neuromuscular fatigue task: an fNIRS study. Advances in experimental medicine and biology. 2013; 789: 73-9.

61. A. M. Do the Warriors Owe Some of Their Success to These "Brain- Zapping" Headphones?. New York, : NY: Complex; 2016.

62. Falcone B, Parasuraman R. Comparative Effects of First- Person Shooter Video Game Experience and Brain Stimulation on Threat Detection Learning. Proceedings of the Human Factors and Ergonomics Society Annual Meeting. 2012; 56(1): 173-7.

63. Berthelot G, Sedeaud A, Marck A, AnteroJacquemin J, Schipman J, Saulière G et al. Has Athletic Performance Reached its Peak? Sports medicine (Auckland, NZ). 2015; 45(9): 1263-71.

64. Vitor- Costa M, Okuno NM, Bortolotti H, Bertollo M, Boggio PS, Fregni F, et al. Improving Cycling Performance: Transcranial Direct Current Stimulation Increases Time to Exhaustion in Cycling. PloS one. 2015; 10(12): e0144916.

65. Williams PS, Hoffman RL, Clark BC. Preliminary evidence that anodal transcranial direct current stimulation enhances time to task failure of a sustained submaximal contraction. PloS one. 2013; 8(12): e81418.

66. Rattray B, Argus C, Martin K, Northey J, Driller M. Is it time to turn our attention toward central mechanisms for post- exertional recovery strategies and performance? Frontiers in physiology. 2015; 6: 79.

67. Reis J, Schambra HM, Cohen LG, Buch ER, Fritsch B, Zarahn E, et al. Noninvasive cortical stimulation enhances motor skill acquisition over multiple days through an effect on consolidation. Proceedings of the National Academy of Sciences of the United States of America. 2009; 106(5): 1590-5.

68. Dubljević V, Saigle V, Racine E. The rising tide of tDCS in the media and academic literature. Neuron. 2014; 82(4): 731-6.

69. BatumanE.ELECTRIFIEDAdventuresinTranscranial Direct- Current Stimulation. The New Yorker. 2015.

70.WexlerA,HamiltonRH.CrowdsourcedtDCSresearch: feasibleorfanciful? AJOBNeuroscience. 2017;8(1):50-3.

71. Wolf FM. Meta- Analysis. Newbury Park, California1986. Available from: https:// methods.sagepub.com/book/meta- analysis.

72. Cohen J. Statistical Power Analysis for the Behavioral Sciences. 2nd edition ed: Routledge; 1988.

73. Lakens D. Calculating and reporting effect sizes to facilitate cumulative science: a practical primer for t-tests and ANOVAs. Frontiers in Psychology. 2013; 4(863).

74. Jain S, Sharma S, Jain K. Meta- Analysis of Fixed, Random and Mixed Effects Models. International Journal of Mathematical, Engineering and Management Sciences. 2019; 4: 199-218.

75. Wertheim J, Colzato LS, Nitsche MA, Ragni M. Enhancing spatial reasoning by anodal transcranial direct current stimulation over the right posterior parietal cortex. Experimental brain research. 2020; 238(1): 181-92.

76. Grandperrin Y, Grosprêtre S, Nicolier M, Gimenez P, Vidal C, Haffen E, et al. Effect of transcranial direct current stimulation on sports performance for two profiles of athletes (power and endurance) (COMPETE): a protocol for a randomized, crossover, double blind, controlled exploratory trial. Trials. 2020; 21(1): 461.

77. Mesquita PHC, Lage GM, Franchini E, RomanoSilva MA, Albuquerque MR. Bi- hemispheric anodal transcranial direct current stimulation worsens taekwondo- related performance. Human Movement Science. 2019; 66: 578-86.

78. Seidel O, Ragert P. Effects of Transcranial Direct Current Stimulation of Primary Motor Cortex on Reaction Time and Tapping Performance: A Comparison Between Athletes and Non- athletes. Frontiers in human neuroscience. 2019; 13: 103.

79. Lattari E, Vieira LAF, Oliveira BRR, Unal G, Bikson M, de Mello Pedreiro RC, et al. Effects of Transcranial Direct Current Stimulation With Caffeine Intake on Muscular Strength and Perceived Exertion. Journal of strength and conditioning research. 2019;33(5): 1237-43. 


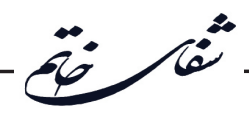

80. Frazer AK, Howatson G, Ahtiainen JP, Avela J, Rantalainen T, Kidgell DJ. Priming the Motor Cortex With Anodal Transcranial Direct Current Stimulation Affects the Acute Inhibitory Corticospinal Responses to Strength Training. Journal of strength and conditioning research. 2019;,33(2):,307-17.

81. Holgado D, Zandonai T, Ciria LF, Zabala M, Hopker J, Sanabria D. Transcranial direct current stimulation (tDCS) over the left prefrontal cortex does not affect time- trial self- paced cycling performance: Evidence from oscillatory brain activity and power output. PloS one. 2019; 14(2): e0210873.

82. Park SB, Sung DJ, KimB, Kim S, Han JK. Transcranial Direct Current Stimulation of motor cortex enhances running performance. PloS one. 2019; 14(2): e0211902.

83. Ciccone AB, Deckert JA, Schlabs CR, Tilden MJ, Herda TJ, Gallagher PM, et al. Transcranial Direct Current Stimulation of the Temporal Lobe Does Not Affect High-Intensity Work Capacity. Journal of strength and conditioning research. 2019; 33(8): 2074-86.

84. Ota K, Shinya M, Kudo K. Transcranial Direct Current Stimulation Over Dorsolateral Prefrontal Cortex Modulates Risk- Attitude in Motor Decision- Making. Frontiers in human neuroscience. 2019; 13(297).

85. Angius L, Santarnecchi E, Pascual- Leone A, Marcora SM. Transcranial Direct Current Stimulation over the Left Dorsolateral Prefrontal Cortex Improves Inhibitory Control and Endurance Performance in Healthy Individuals. Neuroscience. 2019; 419: 34-45.

86. Kamali AM, Nami M, Yahyavi SS, Saadi ZK, Mohammadi A. Transcranial Direct Current Stimulation to Assist Experienced Pistol Shooters in Gaining Even- Better Performance Scores. Cerebellum (London, England). 2019; 18(1): 119-27.

87. Kamali AM, Saadi ZK, Yahyavi SS, Zarifkar A, Aligholi H, Nami M. Transcranial direct current stimulation to enhance athletic performance outcome in experienced bodybuilders. PloS one. 2019; 14(8): e0220363.

88. Huang L, Deng Y, Zheng X, Liu Y. Transcranial Direct Current Stimulation With Halo Sport Enhances Repeated Sprint Cycling and Cognitive Performance. Frontiers in physiology. 2019; 10: 118.

89. Valenzuela PL, Amo C, Sánchez- Martínez G, Torrontegi E, Vázquez- Carrión J, Montalvo Z, et al. Enhancement of Mood but not Performance in Elite Athletes With Transcranial Direct- Current Stimulation. International journal of sports physiology and performance. 2019; 14(3): 310-6.
90. Vargas VZ, Baptista AF, Pereira GOC, Pochini AC, Ejnisman B, Santos MB, et al. Modulation of Isometric Quadriceps Strength in Soccer Players With Transcranial Direct Current Stimulation: A Crossover Study. Journal of strength and conditioning research. 2018; 32(5): 1336-41.

91. Holgado D, Zandonai T, Ciria LF, Zabala M, Hopker J, Sanabria D. tDCS over the left prefrontal cortex does not affect time- trial self- paced cycling performance: Evidence from oscillatory brain activity and power output. bioRxiv. 2018: 341388.

92. Hazime FA, da Cunha RA, Soliaman RR, Romancini ACB, Pochini AC, Ejnisman B, et al. Anodal Transcranial direct current stimulation (TDCS) increases isometric strength of shoulder rotators muscles in handball players. International journal of sports physical therapy. 2017; 12(3) :402-7.

93. Okano AH, Machado DGS, Oliveira Neto L, Farias- Junior LF, Agrícola PMD, Arruda A, et al. Can Transcranial Direct Current Stimulation Modulate Psychophysiological Response in Sedentary Men during Vigorous Aerobic Exercise? International journal of sports medicine. 2017; 38(7): 493-500.

94. Mizuno T, Aramaki Y. Cathodal transcranial direct current stimulation over the $\mathrm{Cz}$ increases joint flexibility. Neuroscience Research. 2017; 114: 55-61.

95. Radel R, Tempest G, Denis G, Besson P, Zory R. Extending the limits of force endurance: Stimulation of the motor or the frontal cortex? Cortex; a journal devoted to the study of the nervous system and behavior. 2017; 97: 96-108.

96. Pixa NH, Steinberg F, Doppelmayr M. Highdefinition transcranial direct current stimulation to both primary motor cortices improves unimanual and bimanual dexterity. Neuroscience letters. 2017; 643: 84-8.

97. Barwood MJ, Butterworth J, Goodall S, House JR, Laws R, Nowicky A, et al. The Effects of Direct Current Stimulation on Exercise Performance, Pacing and Perception in Temperate and Hot Environments. Brain stimulation. 2016; 9(6): 842-9.

98. Angius L, Pageaux B, Hopker J, Marcora SM, Mauger AR. Transcranial direct current stimulation improves isometric time to exhaustion of the knee extensors. Neuroscience. 2016; 339: 363-75.

99. Magalhães Sales M DSC, Vieira Browne RA, Bodnariuc Fontes E, Dos Reis Vieira Olher R, Ernesto C, Herbert G. Transcranial direct current stimulation improves muscle isokinetic performance of young trained individuals. Med Sport. 2016; 69(2): 163-72.

100. Choe J, Coffman BA, Bergstedt DT, Ziegler 
MD, Phillips ME. Transcranial Direct Current Stimulation Modulates Neuronal Activity and Learning in Pilot Training. (1662-5161 (Print)).

101.Hendy AM,TeoWP,KidgellDJ.AnodalTranscranial Direct Current Stimulation Prolongs the Cross- education of Strength and Corticomotor Plasticity. Medicine and science in sports and exercise. 2015; 47(9): 1788-97.

102. Montenegro RA, Okano A, Gurgel J, Porto F, Da Cunha F, Massaferri R, et al. Motor cortex tDCS does not improve strength performance in healthy subjects. Motriz Journal of Physical Education. 2015; 21: 185-93.

103. Montenegro RA, Farinatti P, Lima P, Okano A, Meneses A, De Oliveira Neto L, et al. Motor cortex tDCS does not modulate perceived exertion within multiple- sets of resistance exercises. Isokinetics and exercise science. 2016: 17-24.

104. Angius L, Hopker JG, Marcora SM, Mauger AR. The effect of transcranial direct current stimulation of the motor cortex on exercise- induced pain. European journal of applied physiology. 2015; 115(11): 2311-9.

105. Hendy AM, Kidgell DJ. Anodal tDCS applied during strength training enhances motor cortical plasticity. Medicine and science in sports and exercise. 2013; 45(9): 1721-9.

106. Lampropoulou SI, Nowicky AV. The effect of transcranial direct current stimulation on perception of effort in an isolated isometric elbow flexion task. Motor control. 2013; 17(4): 412-26.

107. Arastoo. A, Zahednejad. Sh, Parsaei. S, Alboghebish.S . The effect of transcranial Direct Current Stimulation on anxiety in Veteran and Disabled Athletes. Medical Journal of Mashhad University of Medical Sciences. 2020; 63(3): 2278-86.

108. Arabi. M, Fardin. M. Effect of a Primary Motor Cortex Transcranial Direct Current Simultion Session on Postural Control of Disabled Athletes with Lower Limb Amputees. Scientific Journal of Rehabilitation Medicine. 2020; 9(2): 277-86.

109. Yousef Moghadas T, Meysam Yavari K, Shahnaz S. Effects of a Single Session Transcranial Direct Current Stimulation (tDCS) on Hand Mental Rotation and Visuo- Spatial Working Memory. Journal of Neuropsychology. 2019; 5(16): 37-54.

110. Arastoo AA, Parsaei S, Sh Z, Alboghebish S, Burbur A. Effect of Unilateral Transcranial Direct Current Stimulation on Reaction Time in Veterans and Athletes with Disabilities. Iranian Journal of War and Public Health. 2019; 11(44): 133-8.
111. Yavari.M.K, Yousef Moghadas T, Shahnaz S, Gharayagh.H.Z,Behjame.F.Effects of Transcranialdirect current stimulation on Imagery ability in students. Journal of Applied Psycology Research. 2018; 9(1): 149-65.

112. Delfani. M, Arabi. M. Investigation of Improving Postural Control Kinetic Parameters in Martial Art Athletes after applying tDCS. Development \& Motor Learning. 2019; 10(34): 587-602.

113. Arastoo. A, Zahednejad. Sh, Parsaei. S, Alboghebish.S, Ataei. N, Ameriasi. S. The effect of direct current stimulation in left dorsolateral prefrontal cortex on working memory in veterans and disabled athletes. Daneshvar Medicine. 2019; 26(139): 25-32.

114. Abedzadeh. A, Alboghebish.S. The Effect of Transcranial Direct Current Stimulus on Selective Attention in Dual Task Paradigm. Journal of Applied Psycology Research. 2017; 8(3): 1-14.

115.HaidarAliH,Ganji.K,Omidfar.A.TheMeta-Analysis of the Effectiveness of Life Skills Training on Mental Health. Developmental Pscychology. 2013; 10(37): 39.

116. Siadatian SH, Ghamarani A, Yaghobian F. The Meta- Analysis of the Effectiveness of Psychological Interventions on the Iranians' Feeling of Happiness. Transformational Psychology: Iranian Psychologists. 2013; 10(37): 61-9.

117. Taylor JL, Gandevia SC. A comparison of central aspects of fatigue in submaximal and maximal voluntary contractions. Journal of applied physiology (Bethesda, Md: 1985). 2008; 104(2): 542-50.

118. Taylor JL, Amann M, Duchateau J, Meeusen R, Rice CL. Neural Contributions to Muscle Fatigue: From the Brain to the Muscle and Back Again. Medicine and science in sports and exercise. 2016; 48(11): 2294-306.

119. Stepniewska I, Preuss TM, Kaas JH. Thalamic connections of the primary motor cortex (M1) of owl monkeys. The Journal of comparative neurology. 1994; 349(4): 558-82.

120. Vaseghi B, Zoghi M, Jaberzadeh S. Does anodal transcranial direct current stimulation modulate sensory perception and pain? A metaanalysis study. Clinical neurophysiology: official journal of the International Federation of Clinical Neurophysiology. 2014; $125(9)$ : $1847-58$.

121. Mauger AR. Fatigue is a pain- the use of novel neurophysiological techniques to understand the fatiguepain relationship. Frontiers in physiology. 2013; 4: 104.

122. Robertson CV, Marino FE. A role for the 


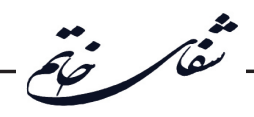

prefrontal cortex in exercise tolerance and termination. Journal of applied physiology (Bethesda, Md: 1985). 2016; 120(4): 464-6.

123. Van Cutsem J, Marcora S, De Pauw K, Bailey S, Meeusen R, Roelands B. The Effects of Mental Fatigue on Physical Performance: A Systematic Review. 2017; 47(8): 1569-88.

124. Rupp T, Perrey S. Prefrontal cortex oxygenation and neuromuscular responses to exhaustive exercise. European journal of applied physiology. 2008; 102(2): 153-63.

125. Rooks CR, Thom NJ, McCully KK, Dishman RK. Effects of incremental exercise on cerebral oxygenation measured by near- infrared spectroscopy: a systematic review. Progress in neurobiology. 2010; 92(2): 134-50.

126. Oppenheimer SM, Gelb A, Girvin JP, Hachinski VC. Cardiovascular effects of human insular cortex stimulation. Neurology. 1992; 42(9): 1727-32.

127. Napadow V, Dhond R, Conti G, Makris N, Brown EN, Barbieri R. Brain correlates of autonomic modulation: combining heart rate variability with fMRI. NeuroImage. 2008; 42(1): 169-77.

128. Montenegro RA, Farinatti Pde T, Fontes EB, Soares PP, Cunha FA, Gurgel JL, etal. Transcranial direct current stimulation influences the cardiac autonomic nervous control. Neuroscience letters. 2011; 497(1): 32-6.

129. López-Alonso V, Fernández- Del- Olmo M, Costantini A, Gonzalez- Henriquez JJ, Cheeran B. Intraindividualvariabilityintheresponsetoanodaltranscranial direct current stimulation. Clinical neurophysiology: official journal of the International Federation of Clinical Neurophysiology. 2015; 126(12): 2342-7.

130. Miranda PC, Mekonnen A, Salvador R, Ruffini $\mathrm{G}$. The electric field in the cortex during transcranial current stimulation. NeuroImage. 2013; 70: 48-58.

131. Frazer AK, Williams J, Spittle M, Kidgell DJ. Cross- education of muscular strength is facilitated by homeostatic plasticity. European journal of applied physiology. 2017; 117(4): 665-77.

132. Zénon A, Sidibé M, Olivier E. Disrupting the supplementarymotorareamakesphysicaleffortappearless effortful. The Journal of neuroscience: the official journal of the Society for Neuroscience. 2015; 35(23): 8737-44.

133. de Morree HM, Klein C, Marcora SM. Perception of effort reflects central motor command during movement execution. Psychophysiology. 2012; 49(9): 1242-53.

134. Wiethoff S, Hamada M, Rothwell JC. Variability in response to transcranial direct current stimulation of the motor cortex. Brain stimulation. 2014; 7(3): 468-75.

135. Madhavan S, Sriraman A, Freels S. Reliability and Variability of tDCS Induced Changes in the Lower Limb Motor Cortex. Brain Sci. 2016; 6(3).

136. Faridnia M, Shojaei M, Rahimi A. The effect of neurofeedback training on the anxiety of elite female swimmers. AnnalsofBiologicalResearch.2012;3:1020-8.

137. Raymond J, Sajid I, Parkinson LA, Gruzelier JH. Biofeedback and dance performance: a preliminary investigation. Applied psychophysiology and biofeedback. 2005; 30(1): 64-73.

138. Rostami R, Sadeghi H, Karami KA, Abadi MN, Salamati P. The Effects of Neurofeedback on the Improvement of Rifle Shooters' Performance. Journal of Neurotherapy. 2012; 16(4): 264-9.

139. Wagner T, Fregni F, Fecteau S, Grodzinsky A, Zahn M, Pascual- Leone A. Transcranial direct current stimulation: a computer-based human model study. NeuroImage. 2007; 35(3): 1113-24.

140. Button KS, Ioannidis JP, Mokrysz C, Nosek BA, Flint J, Robinson ES, et al. Power failure: why small sample size undermines the reliability of neuroscience. Nature reviews Neuroscience. 2013; 14(5): 365-76.

141. Kessler SK, Turkeltaub PE, Benson JG, Hamilton RH. Differences in the experience of active and sham transcranial direct current stimulation. Brain stimulation. 2012; 5(2): 155-62.

142. Fonteneau C, Mondino M, Arns M, Baeken C, Bikson M, Brunoni AR, et al. Sham tDCS: A hidden source of variability? Reflections for further blinded, controlled trials. Brain stimulation. 2019; 12(3): 668-73. 